Universidade de São PaUlo

Faculdade de Economia, Administração e Contabilidade de Ribeirão Preto Departamento de ECONOMIA

Programa de Pós-Graduação em Economia - Área: EConomia Aplicada

IGOR FERREIRA BATISTA MARTINS

Previsão de variáveis macroeconômicas pelo modelo de fatores dinâmicos: uma abordagem por componentes principais esparsas

Orientador: Prof. Dr. Márcio Poletti Laurini

Ribeirão Preto 
Prof. Dr. Vahan Agopyan

Reitor da Universidade de São Paulo

Prof. Dr. André Lucirton Costa

Diretor da Faculdade de Economia, Administração e Contabilidade de Ribeirão Preto

Prof. Dr. Sérgio Kannebley Júnior

Chefe do Departamento de Economia

Prof. Dr. Sérgio Naruhiko Sakurai

Coordenador do Programa de Pós-Graduação em Economia 
IGOR FERREIRA BATISTA MARTINS

\title{
Previsão de variáveis macroeconômicas pelo modelo de fatores dinâmicos: uma abordagem por componentes principais esparsas
}

\author{
Dissertação de mestrado apresentada ao Pro- \\ grama de Pós-Graduação em Economia - Área: \\ Economia Aplicada da Faculdade de Econo- \\ mia, Administração e Contabilidade de Ribei- \\ rão Preto da Universidade de São Paulo para \\ obtenção do título de Mestre em Ciências.
}

Orientador: Prof. Dr. Márcio Poletti Laurini

\section{Versão Corrigida}

(versão original disponível na Faculdade de Economia, Administração e Contabilidade de Ribeirão Preto)

Ribeirão Preto 
Autorizo a reprodução e divulgação total ou parcial deste trabalho, por qualquer meio convencional ou eletrônico, para fins de estudo e pesquisa, desde que citada a fonte.

Martins, Igor Ferreira Batista Martins.

Previsão de variáveis macroeconômicas pelo modelo de fatores dinâmicos: uma abordagem por componentes principais esparsas / Igor Ferreira Batista Martins - Ribeirão Preto, SP, 2020.

119 p.: il.; $30 \mathrm{~cm}$

Dissertação de mestrado apresentada ao Programa de PósGraduação em Economia - Área: Economia Aplicada da Faculdade de Economia, Administração e Contabilidade de Ribeirão Preto da Universidade de São Paulo, para obtenção do título de Mestre em Ciências. - Universidade de São Paulo

Orientador: Laurini, Márcio Poletti

1. Modelo de Fatores. 2. Previsão. 3. SPCA. 
Aos meus pais. 



\section{AGRADECIMENTOS}

Ao meu orientador, Márcio Poletti Laurini, por todo o conhecimento transferido durante o mestrado e pela ótima orientação.

Aos membros da banca por terem aceitado o convite para participar.

Aos professores Sérgio Kannebley Júnior e Fernando Antônio de Barros Júnior pelos comentários nas etapas de qualificação e pré-defesa.

Aos participantes do XIX Encontro Brasileiro de Finanças, da 18 ${ }^{\mathrm{a}}$ ESTE - Escola de Séries Temporais e Econometria e do $41^{\circ}$ Encontro Brasileiro de Econometria pelos comentários realizados.

Aos membros do CEPER e aos professores da FEA-RP que auxiliaram na minha formação.

À minha família pelo incentivo durante os meus estudos.

Ao CNPq pelo apoio financeiro ao longo do mestrado. 



\section{RESUMO}

MARTINS, I. F. B. Previsão de variáveis macroeconômicas pelo modelo de fatores dinâmicos: uma abordagem por componentes principais esparsas. Dissertação (Mestrado) - Faculdade de Economia, Administração e Contabilidade de Ribeirão Preto, Universidade de São Paulo, Ribeirão Preto, 2020.

Modelos fatoriais são um dos principais métodos de previsão macroeconômicas. Tradicionalmente, esses modelos utilizam a matriz de covariância amostral em conjunto da análise de componentes principais. Nesse trabalho, nós analisamos o impacto da escolha do tipo de matriz de covariância e do método de extração dos fatores latentes para previsões macroeconômica decorrentes de modelos fatoriais. A existência de erros de medida nas séries temporais pode introduzir uma estrutura de dependência temporal sendo adequado substituir a matriz de covariância amostral por matrizes de longo prazo. Adicionalmente, a matriz de covariância amostral pode acumular erros de estimação devido ao número elevado de parâmetros a serem estimados. Ao usar um hard-threshold, podemos mitigar esse problema. Já o uso de componentes principais esparsas nos permite usar o tradeoff entre viés e variância para obter estimativas mais precisas em amostras finitas. A metodologia proposta é aplicada a base de dados macroeconômicos apresentada em Stock e Watson (2009). Os resultados obtidos fortalecem a evidência de que usar um subconjunto das séries temporais disponibilizadas para a extração dos fatores não leva, necessariamente, a um pior desempenho preditivo. Similarmente a Kristensen (2017), encontramos que as previsões obtidas por PCA e SPCA são próximas e, adicionalmente, mostramos que o Model Confidence Set não rejeita a hipótese de mesma habilidade preditiva em muitos casos. Por fim, expandimos a evidência apresentada em Smeekes e Wijler (2018) de que modelos que utilizam esparsidade podem levar a um melhor desempenho do que modelos fatoriais baseados na matriz de covariância amostral e PCA quando temos a presença de erros de especificação.

Palavras-chave: Modelo de Fatores. Previsão. SPCA. 



\begin{abstract}
MARTINS, I. F. B.Macroeconomic forecasting with dynamic factor model: a sparse principal components approach. Dissertation (Master Degree) - School of Economics, Business and Accounting at Ribeirão Preto, University of São Paulo, Ribeirão Preto, 2020.

Factor models are one of the main methods used in macroeconomic forecasting. Usually, these models use the sample covariance matrix in conjunction with principal component analysis. In this work, we analyze the impact of the choice of the covariance matrix class and the latent factor extraction method for macroeconomic forecasting based on factor models. The existence of measurement errors in the time series can introduce a time dependency structure, being appropriate to replace the sample covariance matrix with long-run covariance matrices. Additionally, the sample covariance matrix can accumulate estimation errors due to the high number of parameters to be estimated. By using a hard-threshold, we can mitigate this problem. The use of sparse principal components allows us to use the tradeoff between bias and variance to obtain more accurate estimates in finite samples. We apply the proposed methodology to the macroeconomic database presented in Stock e Watson (2009). The results obtained strengths the evidence that using a subset of the dataset for factor extraction does not necessarily lead to worse predictive performance. Similarly to Kristensen (2017), we find that the forecasts based on PCA and SPCA are close and, in addition, we show that the Model Confidence Set does not reject the hypothesis of the equal predictive ability in many cases. Finally, we expand the evidence presented in Smeekes e Wijler (2018) that sparse models can lead to better performance than factor models based on the sample covariance matrix and PCA when there are specification errors.
\end{abstract}

Keywords: Factor Model. Forecast. SPCA. 



\section{LISTA DE ILUSTRAÇÕES}

Figura 5.1 - Número de fatores sugeridos pelos critérios apresentados em Ahn e Horenstein (2013), Bai e Ng (2002) e Onatski (2010) quando modelamos a dependência entre a séries por meio da matriz de covariância amostral . 52

Figura 5.2 - Número de variáveis não nulas sugeridas pelos critérios de Kristensen (2017) e Wang, Li e Leng (2009) quando modelamos a dependência entre a séries por meio da matriz de covariância amostral . . . . . . . . . . . . 53

Figura 5.3 - Número de fatores sugeridos pelos critérios apresentados em Ahn e Horenstein (2013), Bai e Ng (2002) e Onatski (2010) quando modelamos a dependência entre a séries por meio da matriz de covariância de longo prazo proposta em Andrews (1991) . . . . . . . . . . . . . . . . . . . 54

Figura 5.4 - Número de variáveis não nulas sugeridas pelos critérios de Kristensen (2017) e Wang, Li e Leng (2009) quando modelamos a dependência entre a séries por meio da LRCM proposta por Andrews (1991). . . . . . . . . . . . . 54

Figura 5.5 - Número de fatores sugeridos pelos critérios apresentado em Ahn e Horenstein (2013),Bai e Ng (2002) e Onatski (2010) quando modelamos a dependência entre a séries por meio da matriz de covariância de longo prazo proposta em Kiefer e Vogelsang (2002) . . . . . . . . . . . . . . . 56

Figura 5.6 - Número de variáveis não nulas sugeridas pelos critérios de Kristensen (2017) e Wang, Li e Leng (2009) quando modelamos a dependência entre a séries por meio da matriz de covariância da classe fixed $b$ proposta por Kiefer e Vogelsang (2002). . . . . . . . . . . . . . . . . . . . . 56

Figura 5.7 - Número de fatores sugeridos pelos critérios apresentados em Ahn e Horenstein (2013), Bai e Ng (2002) e Onatski (2010) quando modelamos a dependência entre a séries por meio da matriz de covariância após a aplicação de um limiar como proposto em Bickel, Levina et al. (2008). . . . . . . . 57 
Figura 5.8 - Número de variáveis não nulas sugeridas pelos critérios de Kristensen (2017) e Wang, Li e Leng (2009) quando modelamos a dependência entre a séries por meio da matriz de covariância após a aplicação de um limiar como proposto em Bickel, Levina et al. (2008). . . . . . . . . . . . . . . . 58

Figura 5.9 - Número de fatores sugeridos pelos critérios apresentados em Ahn e Horenstein (2013),Bai e Ng (2002) e Onatski (2010) quando modelamos a dependência entre a séries por meio da matriz de covariância proposta em Ledoit e Wolf (2004) . . . . . . . . . . . . . . . . . . . . . . . . 59

Figura 5.10-Previsão do GDP251 para os próximos 1,2,4 e 8 trimestres considerando os modelos do caso $1 . \ldots \ldots \ldots \ldots$. . . . . . . . . . . . . 62

Figura 5.11-Previsão do GDP251 para os próximos 1,2,4 e 8 trimestres considerando os modelos do caso $2 . \ldots \ldots \ldots \ldots \ldots \ldots$

Figura 5.12-Previsão do GDP251 para os próximos 1,2,4 e 8 trimestres considerando os modelos do caso $3 . \ldots \ldots \ldots \ldots \ldots$. . . . . . . . . . . . 65

Figura 5.13-Previsão do GDP251 para os próximos 1,2,4 e 8 trimestres considerando os modelos do caso 4 .

Figura 5.14-Previsão do GDP251 para os próximos 1,2,4 e 8 trimestres considerando os modelos do caso 5 .

Figura 5.15-Previsão do IPS10 para os próximos 1,2,4 e 8 trimestres considerando os modelos do caso 1 .

Figura 5.16-Previsão do IPS10 para os próximos 1,2,4 e 8 trimestres considerando os modelos do caso $2 . \ldots \ldots \ldots \ldots \ldots \ldots$. . . . . . . . . . . . . . . . . .

Figura 5.17-Previsão do IPS10 para os próximos 1,2,4 e 8 trimestres considerando os modelos do caso $3 . \ldots \ldots \ldots \ldots \ldots$. . . . . . . . . . . . . . . . . .

Figura 5.18-Previsão do IPS10 para os próximos 1,2,4 e 8 trimestres considerando os modelos do caso $4 . \ldots \ldots \ldots \ldots \ldots \ldots$

Figura 5.19-Previsão do IPS10 para os próximos 1,2,4 e 8 trimestres considerando os modelos do caso 5 . 
Figura 5.20-Previsão do LHUR para os próximos 1,2,4 e 8 trimestres considerando os modelos do caso $1 . \ldots \ldots \ldots$. . . . . . . . . . . 80

Figura 5.21-Previsão do LHUR para os próximos 1,2,4 e 8 trimestres considerando os modelos do caso $2 . \ldots \ldots \ldots \ldots \ldots$

Figura 5.22-Previsão do LHUR para os próximos 1,2,4 e 8 trimestres considerando os modelos do caso $3 . \ldots \ldots \ldots \ldots$. . . . . . . . . . . . . 84

Figura 5.23-Previsão do LHUR para os próximos 1,2,4 e 8 trimestres considerando os modelos do caso $4 . \ldots \ldots \ldots \ldots \ldots \ldots \ldots$

Figura 5.24-Previsão do LHUR para os próximos 1,2,4 e 8 trimestres considerando os modelos do caso 5.

Figura 5.25-Previsão do CPIAUCSL para os próximos 1,2,4 e 8 trimestres considerando os modelos do caso 1 .

Figura 5.26-Previsão do CPIAUCSL para os próximos 1,2,4 e 8 trimestres considerando os modelos do caso $2 \ldots \ldots \ldots \ldots \ldots \ldots \ldots$

Figura 5.27-Previsão do CPIAUCSL para os próximos 1,2,4 e 8 trimestres considerando os modelos do caso $3 \ldots \ldots \ldots$. . . . . . . . . . . . . . . 92

Figura 5.28-Previsão do CPIAUCSL para os próximos 1,2,4 e 8 trimestres considerando os modelos do caso $4 \ldots \ldots \ldots$. . . . . . . . . . . . . . . 94

Figura 5.29-Previsão do CPIAUCSL para os próximos 1,2,4 e 8 trimestres considerando os modelos do caso $5 \ldots \ldots \ldots \ldots \ldots \ldots$

Figura 5.30-Previsão do FSPCOM para os próximos 1,2,4 e 8 trimestres considerando os modelos do caso 1 .

Figura 5.31-Previsão do FSPCOM para os próximos 1,2,4 e 8 trimestres considerando os modelos do caso 2.

Figura 5.32-Previsão do FSPCOM para os próximos 1,2,4 e 8 trimestres considerando os modelos do caso $3 . \ldots \ldots$. . . . . . . . . . . . . . . . . 102

Figura 5.33-Previsão do FSPCOM para os próximos 1,2,4 e 8 trimestres considerando os modelos do caso 4. 
Figura 5.34-Previsão do FSPCOM para os próximos 1,2,4 e 8 trimestres considerando os modelos do caso $5 . \ldots \ldots$. . . . . . . . . . . . . . . . 104 


\section{LISTA DE TABELAS}

Tabela 5.1 - Classificação das séries temporais contidas na base de dados apresentada em Stock e Watson (2009). . . . . . . . . . . . . . . . . . . . . . 49

Tabela 5.2 - Número de séries temporais presentes em cada um dos grupos após a remoção das 35 séries realizadas em Stock e Watson (2009). . . . . . . . . . 50

Tabela 5.3 - Descrição das séries a serem previstas e sua sigla correspondente. . . . . . 51

Tabela 5.4 - Modelos usados para previsão quando consideramos que a dependência entre séries é modelada pela matriz de covariância amostral . . . . . . . . . 52

Tabela 5.5 - Modelos usados para previsão quando consideramos que a dependência entre séries é modelada pela LRCM proposta por Andrews (1991). . . . . . 55

Tabela 5.6 - Modelos usados para previsão quando consideramos que a dependência entre séries é modelada pela LRCM proposta por Kiefer e Vogelsang (2002). 55

Tabela 5.7 - Modelos usados para previsão quando consideramos que a dependência entre séries é modelada pela matriz de covariância após a aplicação de um hard-threshold. . . . . . . . . . . . . . . . . . . . 58

Tabela 5.8 - DFM estimados na aplicação empírica. . . . . . . . . . . . . . . . . . 60

Tabela 5.9 - Modelos adaLASSO, LASSO e GDFM estimados na aplicação empírica. 60

Tabela 5.10-Modelo com o menor MSFE para cada horizonte de previsão quando consideramos apenas os modelos que usam a matriz de covariância amostral e o método de extração é PCA ou SPCA com número fixo de variáveis não nulas. 61

Tabela 5.11-Resultados do MCS para GDP251 em cada horizonte de previsão quando consideramos apenas os modelos que usam a matriz de covariância amostral e o método de extração é PCA ou SPCA com número fixo de variáveis

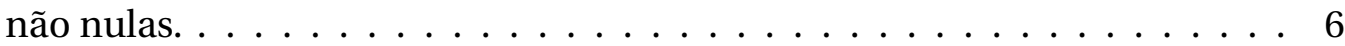

Tabela 5.12-Modelo com o menor MSFE para cada horizonte de previsão quando adicionamos a possibilidade de modelar as séries pelas matrizes de Andrews e Fixed-b e o método de extração é PCA ou SPCA com número fixo de variáveis não nulas. . . . . . . . . . . . . . . . . . 6 
Tabela 5.13-MCS para cada horizonte de previsão quando adicionamos a possibilidade de modelar as séries pelas matrizes de Andrews e Fixed-b e o método de extração é PCA ou SPCA com número fixo de variáveis não nulas. . . . . . . . 64

Tabela 5.14-Modelo com o menor MSFE para cada horizonte de previsão quando adicionamos ao caso anterior a possibilidade de modelar as séries por matrizes de covariância após a aplicação de um limiar . . . . . . . . . . . . . . . . . 64

Tabela 5.15-MCS para cada horizonte de previsão quando adicionamos ao caso anterior a possibilidade de modelar as séries por matrizes de covariância após a aplicação de um limiar . . . . . . . . . . . . . . . . . . . 66

Tabela 5.16-Modelo para GDP251 com o menor MSFE para cada horizonte de previsão quando consideramos as matrizes de covariância amostral, Andrews, fixedb, matriz após a aplicação de um limiar e o método de extração pode ser

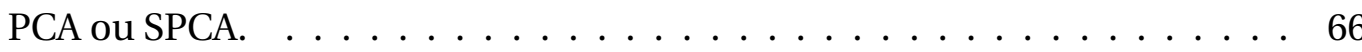

Tabela 5.17-MCS para cada horizonte de previsão quando consideramos as matrizes de covariância amostral, Andrews, fixed-b, matriz após a aplicação de um limiar e o método de extração pode ser PCA ou SPCA. . . . . . . . . . . . . . 68

Tabela 5.18-Modelo com o menor MSFE para cada horizonte de previsão quando consideramos todos os modelos apresentados na seção anterior. . . . . . . . . . 68

Tabela 5.19-MCS para cada horizonte de previsão quando consideramos todos os modelos apresentados na seção anterior. . . . . . . . . . . . . . . . 70

Tabela 5.20-Modelos para o IPS10 com o menor MSFE para cada horizonte de previsão quando consideramos apenas os modelos que usam a matriz de covariância amostral e o método de extração é PCA ou SPCA com número fixo de variáveis não nulas. . . . . . . . . . . . . . . . . . . . . 72

Tabela 5.21-MCS para o IPS10 para cada horizonte de previsão quando consideramos apenas os modelos que usam a matriz de covariância amostral e o método de extração é PCA ou SPCA com número fixo de variáveis não nulas. . . . . 72 
Tabela 5.22-Modelos para IPS10 com o menor MSFE para cada horizonte de previsão quando adicionamos a possibilidade de modelar as séries pelas matrizes de Andrews e Fixed-b e o método de extração é PCA ou SPCA com número fixo de variáveis não nulas. . . . . . . . . . . . . . . . . . . . . . . . . 72

Tabela 5.23-MCS para IPS10 para cada horizonte de previsão quando adicionamos a possibilidade de modelar as séries pelas matrizes de Andrews e Fixed-b e o método de extração é PCA ou SPCA com número fixo de variáveis não nulas. 74

Tabela 5.24-Modelo para IPS10 com o menor MSFE para cada horizonte de previsão quando adicionamos ao caso anterior a possibilidade de modelar as séries por matrizes de covariância após a aplicação de um limiar . . . . . . . . . 74

Tabela 5.25-MCS para IPS10 para cada horizonte de previsão quando adicionamos ao caso anterior a possibilidade de modelar as séries por matrizes de covariância após a aplicação de um limiar . . . . . . . . . . . . . . . . . . 7

Tabela 5.26-Modelo para IPS10 com o menor MSFE para cada horizonte de previsão quando consideramos as matrizes de covariância amostral, Andrews, FixedB, matriz após a aplicação de um limiar e o método de extração pode ser PCA ou SPCA.

Tabela 5.27-MCS para IPS10 para cada horizonte de previsão quando consideramos as matrizes de covariância amostral, Andrews, Fixed-B, matriz após a aplicação de um limiar e o método de extração pode ser PCA ou SPCA. . . . . . . 77

Tabela 5.28-Modelo para IPS10 com o menor MSFE para cada horizonte de previsão quando consideramos todos os modelos apresentados na seção anterior. .

Tabela 5.29-MCS para IPS10 para cada horizonte de previsão quando consideramos todos os modelos apresentados na seção anterior. . . . . . . . . . . . . . .

Tabela 5.30-Modelos para LHUR com o menor MSFE para cada horizonte de previsão quando consideramos apenas os modelos que usam a matriz de covariância amostral e o método de extração é PCA ou SPCA com número fixo de

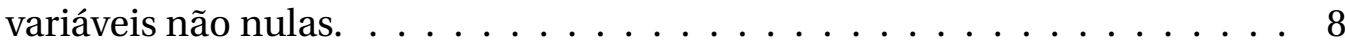


Tabela 5.31-MCS para LHUR para cada horizonte de previsão quando consideramos apenas os modelos que usam a matriz de covariância amostral e o método de extração é PCA ou SPCA com número fixo de variáveis não nulas. . . . . . 81

Tabela 5.32-Modelos para LHUR com o menor MSFE para cada horizonte de previsão quando adicionamos a possibilidade de modelar as séries pelas matrizes de Andrews e Fixed-b e o método de extração é PCA ou SPCA com número fixo de variáveis não nulas. . . . . . . . . . . . . . . . . . . . . . . 8

Tabela 5.33-MCS para LHUR para cada horizonte de previsão quando adicionamos a possibilidade de modelar as séries pelas matrizes de Andrews e Fixed-b e o método de extração é PCA ou SPCA com número fixo de variáveis não nulas. 83

Tabela 5.34-Modelo para LHUR com o menor MSFE para cada horizonte de previsão quando adicionamos ao caso anterior a possibilidade de modelar as séries por matrizes de covariância após a aplicação de um limiar . . . . . . . . . . 83

Tabela 5.35-MCS para LHUR para cada horizonte de previsão quando adicionamos ao caso anterior a possibilidade de modelar as séries por matrizes de covariância após a aplicação de um limiar . . . . . . . . . . . . . . . . . 83

Tabela 5.36-Modelo para LHUR com o menor MSFE para cada horizonte de previsão quando consideramos as matrizes de covariância amostral, Andrews, FixedB, matriz após a aplicação de um limiar e o método de extração pode ser

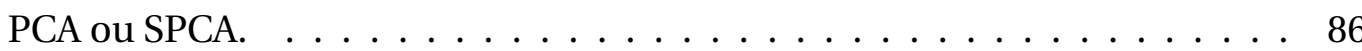

Tabela 5.37-MCS para LHUR para cada horizonte de previsão quando consideramos as matrizes de covariância amostral, Andrews, Fixed-B, matriz após a aplicação de um limiar e o método de extração pode ser PCA ou SPCA. . . . . . . 86

Tabela 5.38-Modelo para LHUR com o menor MSFE para cada horizonte de previsão quando consideramos todos os modelos apresentados na seção anterior. 86

Tabela 5.39-MCS para LHUR para cada horizonte de previsão quando consideramos todos os modelos apresentados na seção anterior. . . . . . . . . . . . . . 88 
Tabela 5.40-Modelos para CPIAUCSL com o menor MSFE para cada horizonte de previsão quando consideramos apenas os modelos que usam a matriz de covariância amostral e o método de extração é PCA ou SPCA com número fixo de variáveis não nulas. . . . . . . . . . . . . . . . . . . . . . . . 90

Tabela 5.41-MCS para CPIAUCSL para cada horizonte de previsão quando consideramos apenas os modelos que usam a matriz de covariância amostral e o método de extração é PCA ou SPCA com número fixo de variáveis não nulas. 90

Tabela 5.42-Modelos para CPIAUCSL com o menor MSFE para cada horizonte de previsão quando adicionamos a possibilidade de modelar as séries pelas matrizes de Andrews e Fixed-b e o método de extração é PCA ou SPCA com número fixo de variáveis não nulas. . . . . . . . . . . . . . . . . . . . . . . 90

Tabela 5.43-MCS para CPIAUCSL para cada horizonte de previsão quando adicionamos a possibilidade de modelar as séries pelas matrizes de Andrews e Fixed-b e o método de extração é PCA ou SPCA com número fixo de variáveis não

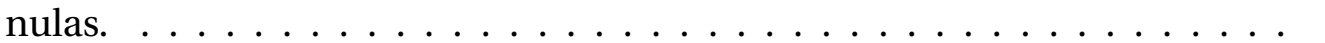

Tabela 5.44-Modelo para CPIAUCSL com o menor MSFE para cada horizonte de previsão quando adicionamos ao caso anterior a possibilidade de modelar as séries por matrizes de covariância após a aplicação de um limiar . . . . . .

Tabela 5.45-MCS para CPIAUCSL para cada horizonte de previsão quando adicionamos ao caso anterior a possibilidade de modelar as séries por matrizes de covariância após a aplicação de um limiar . . . . . . . . . . . . . . . . .

Tabela 5.46-Modelo para CPIAUCSL com o menor MSFE para cada horizonte de previsão quando consideramos as matrizes de covariância amostral, Andrews, Fixed-B, matriz após a aplicação de um limiar e o método de extração pode ser PCA ou SPCA. . . . . . . . . . . . . . . . . . . 95

Tabela 5.47-MCS para CPIAUCSL para cada horizonte de previsão quando consideramos as matrizes de covariância amostral, Andrews, Fixed-B, matriz após a aplicação de um limiar e o método de extração pode ser PCA ou SPCA. . 95 
Tabela 5.48-Modelo para CPIAUCSL com o menor MSFE para cada horizonte de previsão quando consideramos todos os modelos apresentados na seção anterior. 95

Tabela 5.49-MCS para CPIAUCSL para cada horizonte de previsão quando consideramos todos os modelos apresentados na seção anterior. . . . . . . . . . . . . 97

Tabela 5.50-Modelos para FSPCOM com o menor MSFE para cada horizonte de previsão quando consideramos apenas os modelos que usam a matriz de covariância amostral e o método de extração é PCA ou SPCA com número fixo de variáveis não nulas. . . . . . . . . . . . . . . . . . . . . . . 999

Tabela 5.51-MCS para FSPCOM para cada horizonte de previsão quando consideramos apenas os modelos que usam a matriz de covariância amostral e o método de extração é PCA ou SPCA com número fixo de variáveis não nulas. . . . . 99

Tabela 5.52-Modelos para FSPCOM com o menor MSFE para cada horizonte de previsão quando adicionamos a possibilidade de modelar as séries pelas matrizes de Andrews e Fixed-b e o método de extração é PCA ou SPCA com número fixo de variáveis não nulas. . . . . . . . . . . . . . . . . . . . . . . . . 99

Tabela 5.53-MCS para FSPCOM para cada horizonte de previsão quando adicionamos a possibilidade de modelar as séries pelas matrizes de Andrews e Fixed-b e o método de extração é PCA ou SPCA com número fixo de variáveis não nulas.101

Tabela 5.54-Modelo para FSPCOM com o menor MSFE para cada horizonte de previsão quando adicionamos ao caso anterior a possibilidade de modelar as séries por matrizes de covariância após a aplicação de um limiar . . . . . . . . . . 101

Tabela 5.55-MCS para FSPCOM para cada horizonte de previsão quando adicionamos ao caso anterior a possibilidade de modelar as séries por matrizes de covariância após a aplicação de um limiar . . . . . . . . . . . . . . . 101

Tabela 5.56-Modelo para FSPCOM com o menor MSFE para cada horizonte de previsão quando consideramos as matrizes de covariância amostral, Andrews, FixedB, matriz após a aplicação de um limiar e o método de extração pode ser PCA ou SPCA. . . . . . . . . . . . . . . . . . . . 105 
Tabela 5.57-MCS para FSPCOM para cada horizonte de previsão quando consideramos as matrizes de covariância amostral, Andrews, Fixed-B, matriz após a aplicação de um limiar e o método de extração pode ser PCA ou SPCA. . . 105

Tabela 5.58-Modelo para FSPCOM com o menor MSFE para cada horizonte de previsão quando consideramos todos os modelos apresentados na seção anterior. 105

Tabela 5.59-MCS para FSPCOM para cada horizonte de previsão quando consideramos todos os modelos apresentados na seção anterior. . . . . . . . . . . . . . 106 Tabela A.1 - MSFE para todos os modelos estimados na aplicação empírica para o GDP251115 Tabela A.2 - MSFE para todos os modelos estimados na aplicação empírica para o IPS10 116 Tabela A.3 - MSFE para todos os modelos estimados na aplicação empírica para o LHUR117 Tabela A.4 - MSFE para todos os modelos estimados na aplicação empírica para o CPI-

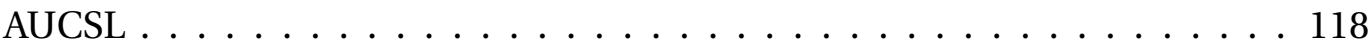

Tabela A.5 - MSFE para todos os modelos estimados na aplicação empírica para o FSP-

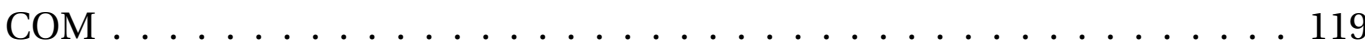




\section{SUMÁRIO}

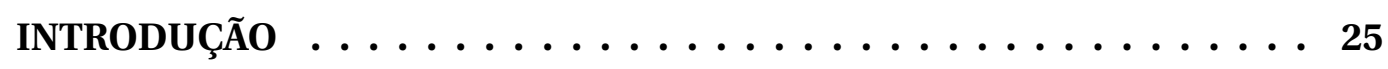

MATRIZES DE COVARIÂNCIA . . . . . . . . . . . . . 29

Matrizes de covariância de longo prazo $\ldots \ldots \ldots \ldots$

$1.2 \quad$ Shrinkage e a matriz de covariância $\ldots \ldots \ldots \ldots \ldots \ldots$

2 COMPONENTES PRINCIPAIS E ESPARSIDADE $\ldots \ldots \ldots \ldots \ldots$

$2.1 \quad$ Análise de componentes principais $\ldots \ldots \ldots \ldots$

$2.2 \quad$ Análise de componentes principais esparsas $\ldots \ldots \ldots \ldots$

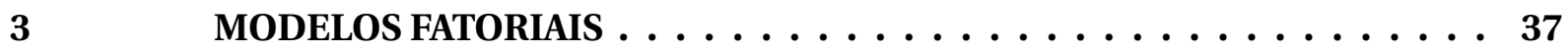

M.1 Modelo de fatores dinâmicos $\ldots \ldots \ldots \ldots$

3.2 Hipóteses de identificação $\ldots \ldots \ldots \ldots \ldots \ldots$

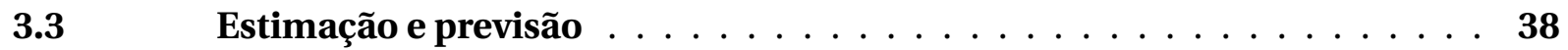

$3.4 \quad$ Número de fatores latentes $\ldots \ldots \ldots \ldots \ldots \ldots \ldots$

3.5 Comparação de previsões $\ldots \ldots \ldots \ldots \ldots \ldots \ldots \ldots \ldots \ldots \ldots$

$4 \quad$ MODELOS CONCORRENTES $\ldots \ldots \ldots \ldots \ldots \ldots \ldots \ldots$

$4.1 \quad$ Regressões com penalização $\ldots \ldots \ldots \ldots \ldots \ldots$

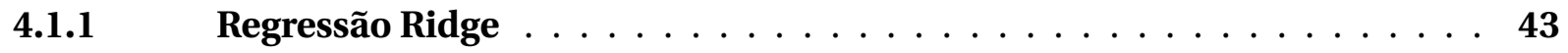

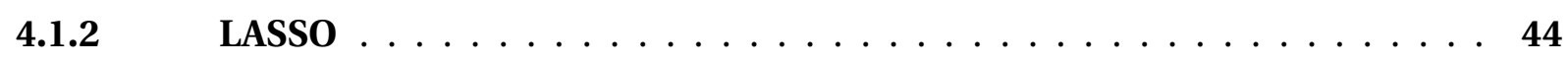

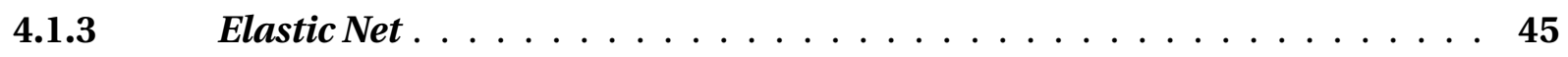

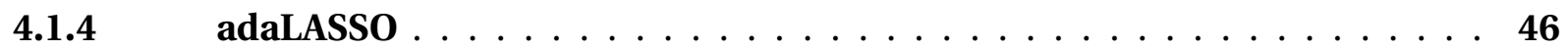

$4.2 \quad$ Generalized Dynamic Factor Model $\ldots \ldots \ldots \ldots$

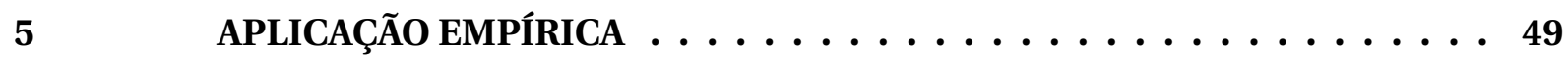

$5.1 \quad$ Base de dados $\ldots \ldots \ldots \ldots \ldots \ldots \ldots \ldots \ldots$

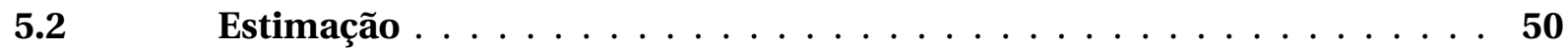

5.2.1 Matriz de covariância amostral $\ldots \ldots \ldots \ldots \ldots \ldots$ 
5.2.2 LRCM de Andrews $\ldots \ldots \ldots \ldots \ldots \ldots \ldots \ldots \ldots$

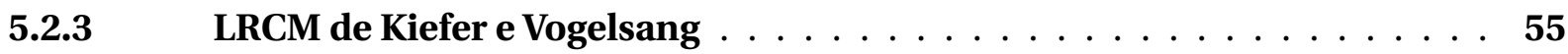

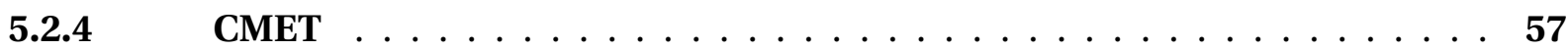

5.2.5 Matriz de Ledoit e Wolf $\ldots \ldots \ldots \ldots \ldots \ldots \ldots$

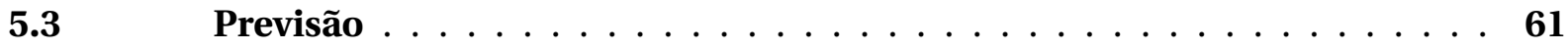

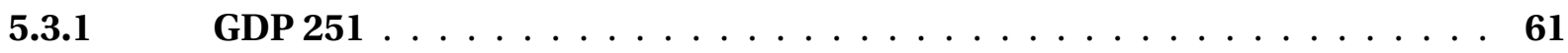

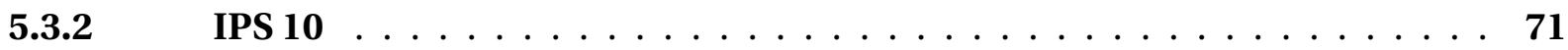

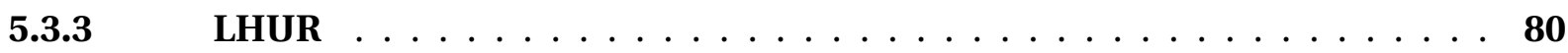

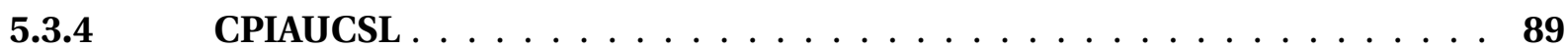

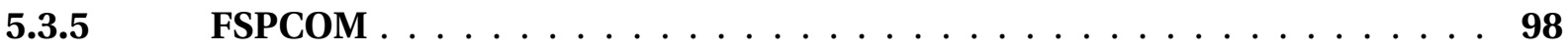

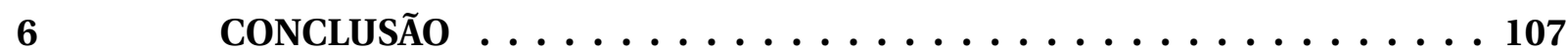

REFERÊNCIAS $\ldots \ldots \ldots \ldots \ldots$

\section{APÊNDICES}

APÊNDICE A - MSFE PARA CADA MODELO ESTIMADO . . . . . . . 115

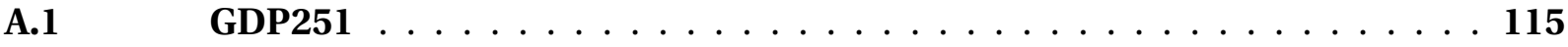

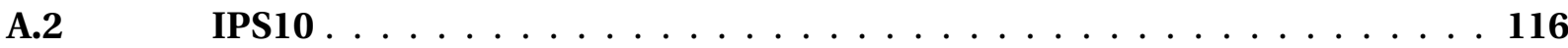

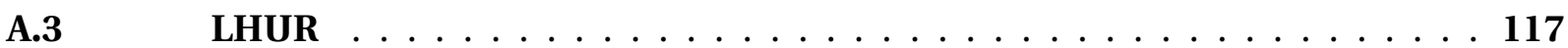

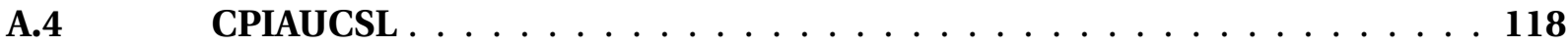

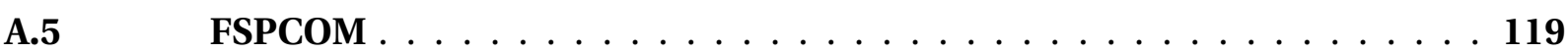





\section{INTRODUÇÃO}

No decorrer da última década, a disponibilização de painéis de dados macroeconômicos com grande quantidade de série temporais tem se tornado cada vez mais comum. Diversos trabalhos consideram um grande número de potenciais preditores com o intuito de obter informações relevantes para melhorar previsões macro-financeiras. Por exemplo, Medeiros, Vasconcelos e Freitas (2016), usa um conjunto de 102 variáveis mensais para prever a inflação brasileira, enquanto Vieira, Fernandes e Chague (2017) usa cerca de 200 variáveis semanais para prever a curva da taxa de juros (yield curve) brasileira. Em muitos casos, o número de séries temporais usadas como possíveis preditores supera a quantidade de pontos no tempo. De acordo com Pourahmadi (2013), nessa situação, é dito que o problema a ser investigado possui alta dimensão.

Nesse contexto, métodos tradicionais, como mínimos quadrados ordinários (Ordinary Least Squares - OLS) e vetores autoregressivos (Vector Autoregressions - VAR) são inadequados para realizar previsões. Tibshirani, Wainwright e Hastie (2015) mostra que a solução obtida por OLS não será única e cada uma das possíveis soluções levará a overfit quase-certamente. Já, por exemplo, Bańbura, Giannone e Reichlin (2008) argumenta que o VAR sofrerá com a maldição da dimensionalidade.

Uma alternativa ao uso de cada uma das séries individualmente, é representar o conjunto de potenciais preditores por um pequeno número de fatores latentes e componentes idiossincráticos, indicando comovimentos e características individuais, respectivamente. Essa representação sugere uma estrutura fatorial. Entre os modelos fatoriais, Stock e Watson (2009) indica o modelo de fatores dinâmicos (Dynamic Factor Model - DFM) como método padrão para previsões macroeconômicas.

Uma das hipóteses tradicionalmente usadas na estimação de modelos fatoriais, e.g. Stock e Watson (2002), é de que a matriz de covariância amostral modela adequadamente a dependência entre as séries macroeconômicas permitindo que os fatores latentes sejam extraídos de forma consistente.

No entanto, como argumentado em Fan e Liao (2019), dados ruidosos podem levar a violação dessa hipótese. Por exemplo, a presença de ruído pode distorcer os autovalores da matriz de covariância amostral dos dados. Adicionalmente, na presença de erros de medida, a primeira diferença pode introduzir componentes MA nas versões estacionárias das séries macroeconômicas. Como apresentado em Laurini e Ohashi (2015), nessas situações, o equivalente amostral da matriz de covariância pode não ser uma representação adequada da dependência entre séries.

Uma vez determinada a estrutura de dependência, podemos usar algum método estatístico para extrair os fatores latentes. Uma das formas mais populares é apresentada em 
Stock e Watson (2002). Neste artigo, Stock e Watson usam análise de componentes principais (Principal Component Analysis - PCA) para recuperar os fatores latentes. A PCA é um método estatístico que permite que variáveis não ortogonais sejam representadas por um conjunto de variáveis sintéticas, construídas por meio de combinações lineares de todo o conjunto de dados, de modo a produzir componentes ortogonais entre si e que capturam a variabilidade dos dados de forma sequencial.

Apesar da popularidade da PCA para recuperar os fatores latentes, e.g. Bai e Ng (2002) e Stock e Watson (2009), artigos como Boivin e Ng (2006), Bai e Ng (2008) e Kim e Swanson (2014) questionam se é benéfico, em termos preditivos, usar a maior quantidade possível de séries macroeconômicas para a estimação dos fatores. A partir de simulação e dados reais, Boivin e Ng (2006) encontra que uma menor quantidade de variáveis não implica em pior desempenho preditivo. Bai e Ng (2008) usa a abordagem de target predictors para indicar que o uso de um subconjunto de série temporais pode levar a melhores previsões que o uso de todas as informações disponíveis. Já Kim e Swanson (2014) usa métodos de redução, como o Elastic Net, para realizar uma pré-seleção das variáveis que compõem os fatores e indica que esse método, em geral, leva a um menor erro quadrático médio de previsão (Mean Squared Forecast Error - MSFE) do que modelos puramente autoregressivos e modelos fatoriais sem a pré-seleção.

Uma outra forma de utilizar apenas um subconjunto dos dados disponíveis é por meio da análise componentes principais esparsas (Sparse Principal Components Analysis SPCA). A SPCA, introduzida por Zou, Hastie e Tibshirani (2006), é uma alternativa a PCA. Ela utiliza um método de shrinkage, como o Least Absolute Shrinkage and Selection Operator (LASSO) ou o Elastic Net, na construção das componentes principais, possibilitando que a matriz de carregamento seja esparsa, ou seja, que muitos dos seus elementos sejam zeros. Essa representação esparsa indica uma seleção das variáveis incluídas na construção dos fatores. Zou, Hastie e Tibshirani (2006) indica que é possível utilizar o tradeoff entre viés e variância para obter estimativas mais precisas, em amostras finitas, do que as alcançadas pela PCA. Portanto, é possível que ocorra ganhos preditivos no modelo.

Como destacado por Kristensen (2017), o método SPCA, com algumas exceções, tem sido pouco explorado para previsão de variáveis macroeconômicas. Kim e Swanson (2014) compara o uso do SPCA em relação ao PCA e PCA após pré-seleção por Elastic Net. Kristensen (2017) faz contribuições teóricas mostrando que o SPCA é capaz de estimar os fatores de forma consistente e discute um critério de informação para obtenção consistente do parâmetro de penalização. Porém, ambos modelam a dependência entre os dados apenas por meio da matriz de covariância amostral, não utilizam o DFM em suas aplicações empíricas e comparam as previsões feitas pelos modelos por meio do MSFE.

Nesse trabalho, analisamos os impactos da escolha da matriz de covariância e do método de extração dos fatores latentes para previsões macroeconômica decorrentes de 
modelos fatoriais.

Considerarmos matrizes de covariância de longo prazo (Long-run covariance matrices LRCM), matrizes de covariância após a aplicação de um limiar (Covariance matrices estimation by thresholding - CMET) e baseadas na combinação convexa entre a matriz de covariância amostral e a matriz identidade. Adicionalmente, estimamos o DFM usando PCA e SPCA para extrair os fatores latentes.

Na aplicação empírica, é utilizada a tradicional base de variáveis macroeconômicas apresentada em Stock e Watson (2009). Essa base é utilizada em vários artigos como Groen e Kapetanios (2016), Aiolfi, Capistrán e Timmermann (2010) e Castle, Clements e Hendry (2013) e, após atualizações, em Stock e Watson (2012) e Koop (2013). Além da comparação do desempenho entre as diferentes especificações de DFM, consideramos métodos baseados em regressão com penalização, como o LASSO, e o modelo de fatores dinâmicos generalizado (Generalized Dynamic Factor Model - GDFM). Para comparar o desempenho preditivo dos modelos, usamos o MSFE e o Model Confidence Set (MCS).

Este trabalho está estruturado em 5 capítulos, além desta introdução, conclusão e apêndices. No Capítulo 1, apresenta-se uma revisão das diferentes matrizes de covariâncias usadas nesse trabalho. No Capítulo 2 são apresentados os métodos PCA e SPCA. No Capítulo 3 , apresenta-se uma breve revisão de modelos fatoriais e de métodos para determinar o número de fatores em cada modelo. No Capítulo 4, apresentamos métodos de alta-dimensão alternativos a DFM. E, por fim, no Capítulo 5 fazemos uma aplicação empírica. 



\section{MATRIZES DE COVARIÂNCIA}

Seja X um matriz $T \times n$ de dados em que T é o tamanho da amostra e n é o número de variáveis. Adicionalmente, considere que as colunas de X estão centradas na média. Uma das formas mais simples de medir a dependência entre variáveis é por meio da matriz de covariância amostral

$$
S=\frac{X^{\prime} X}{T}
$$

De acordo com Laurini e Ohashi (2015), o uso da matriz de covariância amostral é válido quando os processos analisados são fracamente estacionários e independentes. Porém, $S$ não é adequada para modelar a dependência entre séries na presença de alguma forma de dependência temporal causada pela presença de correlação serial ou por um processo contaminante. Adicionalmente, o seu desempenho pode ser ruim no contexto de alta dimensão. Como descrito em Fan, Liao e Liu (2016), se $n>T$, então S será singular. Além disso, $\mathrm{S}$ acumulará erros de estimação devido ao número elevado de parâmetros a serem estimados.

Ambas as situações podem ocorrer no contexto de séries macroeconômicas. Para o problema de dependência temporal, uma alternativa é o uso de LRCM. Para o problema de alta dimensão, consideramos CMET e regularizar a estrutura de autovalor-autovetor de S.

\subsection{Matrizes de covariância de longo prazo}

Considere uma série não estacionária $C_{t}^{o}$

$$
C_{t}^{o}=C_{t}^{v}+u_{t}
$$

em que $C_{t}^{o}$ é o valor observado, $C_{t}^{v}$ representa o valor verdadeiro de C no período t e $u_{t}$ é um erro de medida.

Uma alternativa para combater a não estacionariedade de $C_{t}^{o}$ é trabalhar com a sua primeira diferença

$$
\begin{gathered}
C_{t}^{o}-C_{t-1}^{o}=C_{t}^{v}-C_{t-1}^{v}+u_{t}-u_{t-1} \\
\Delta C_{t}^{o}=\Delta C_{t}^{v}+u_{t}-u_{t-1}
\end{gathered}
$$

Porém, a Equação 1.4 indica que, caso exista um erro de medida associado a uma série não estacionária, a diferenciação introduzirá um componente MA(1). De acordo com 
Laurini e Ohashi (2015), esse erro pode contaminar a estrutura de dependência das séries. Portanto, não seria adequado usar a matriz S para modelar a dependência entre séries. Uma alternativa é usar uma LRCM.

Assim como em Laurini e Ohashi (2015), a LRCM para um processo $w_{t}$ fracamente estacionário e ergódico é definida como

$$
V_{l r}=\sum_{j=-\infty}^{\infty} \gamma(j)
$$

em que $\gamma(j)$ representa a autocovariância de ordem $\mathrm{j}$.

Apesar de ser possível calcular $\hat{\gamma}(j)$ para cada j, o equivalente amostral de $\hat{V}_{l r}$ requer o cálculo de infinitas autocovariâncias amostrais.

Uma das formas de contornar essa dificuldade é utilizar a estimação não paramétrica proposta por Andrews (1991). Essa abordagem baseia-se em estimar

$$
V_{l r}^{A}=\sum_{j=-(n-1)}^{n-1} K\left(\frac{j}{b}\right) \gamma(j)
$$

em que $K(\cdot)$ é uma função de kernel contínua, simétrica e tal que $K(0)=1$. Para que seja garantida a consistência do estimador, é necessário que o tamanho da janela cresça a uma taxa menor do que o tamanho da amostra. Portanto, a regra de janela (bandwidth) b é determinada de modo que $b \rightarrow \infty$ a medida que $n \rightarrow \infty$.

Apesar da classe de estimadores propostos por Andrews (1991) serem consistentes, Müller (2007) mostra que eles não são robustos a erros de medida. A não robustez a erros de medidas motiva o uso de estimadores inconsistentes, mas que são robustos a contaminações, como os apresentados em Kiefer e Vogelsang (2002) e Kiefer e Vogelsang (2005). Em particular, Kiefer e Vogelsang (2002) sugere o uso de toda a amostra como regra de janela. Como o tamanho da janela não varia com a amostra, essa classe de estimadores, conhecida como fixed $b$, é claramente inconsistente.

Além de ser robusto a contaminações, o estimador proposto por Kiefer e Vogelsang (2002) possui um bom desempenho em amostras finitas.

Assim como mostrado em Laurini e Ohashi (2015), uma versão do estimador da classe fixed $b$ é representada por

$$
V_{l r}^{K V}=\frac{1}{T} \sum_{i=1}^{T} \sum_{j=1}^{T} \hat{w}_{i}\left(1-\frac{|i-j|}{T}\right) \hat{w}_{j}
$$

em que $\hat{w}$ representa o processo $w_{t}$ centrado na média e a função de kernel é do tipo Bartlett. 


\subsection{Shrinkage e a matriz de covariância}

Além dos problemas relativos a dependência temporal, S pode não ser adequada para modelar a dependência entre séries pois acumulará erros de estimação devido ao número elevado de parâmetros a serem estimados. Como em Bickel, Levina et al. (2008), uma alternativa é substituir S pela CMET usando um hard-threshold.

Considere uma matriz $\mathrm{M}$ em que cada um de seus elementos são representados por $m_{i j}$. O operador de hard-threshold $\mathrm{H}(\cdot)$ é tal que

$$
H(M, s)=m_{i j} \mathbb{1}\left(\left|m_{i j}\right| \geq s\right)
$$

em que $\mathbb{1}$ é a função indicadora e s é um limiar. No caso em que substituímos M por S, restringimos a aplicação do operador $\mathrm{H}(\cdot)$ apenas aos elementos fora da diagonal principal. Assim como descrito em Bickel, Levina et al. (2008), o limiar s pode ser escolhido por meio de um processo de validação cruzada.

A partir da aplicação do operador H(·), podemos evitar a estimação das covariâncias com valor absoluto menor que um determinado limiar ao atribuir zero para essas entradas da matriz. Portanto, muitos elementos da matriz podem ser reduzidos a zero. Logo, o operador de hard-threshold é um método de shrinkage capaz de introduzir esparsidade na matriz de covariância.

Porém, além do acúmulo de erros de estimações, Pourahmadi (2013) indica que a alta dimensão pode levar a distorções na estrutura de autovalores - autovetores de S quando comparada a sua contra-parte amostral. Por exemplo, Ledoit e Wolf (2004) mostra que o maior autovalor de $\mathrm{S}$ tende a ser maior que sua contra-parte amostral, enquanto o menor autovalor de $S$ tende a ser menor que sua contra-parte amostral.

Uma alternativa capaz de mitigar essa distorção é impor uma regularização nos autovalores como a sugerida no próprio artigo Ledoit e Wolf (2004). Abaixo será utilizada a mesma notação de Ledoit e Wolf (2004).

Note que S é um estimador não viesado para a matriz de covariância populacional $\Sigma$. Porém, S é instável quando $n>>T$. Note também que a matriz identidade é extremamente estável, porém pode ser uma estimativa fortemente viesada de $\Sigma$.

Uma alternativa é considerar estimadores na forma

$$
\Sigma^{*}=\alpha_{1} I+\alpha_{2} S
$$

e escolher os coeficientes $\alpha_{1}$ e $\alpha_{2}$ de forma a minimizar uma função de perda. Note que toda a perda atribuída a I ocorre por conta do viés, já a perda relativa a S ocorre por conta da variância. Portanto, a escolha de $\alpha_{1}$ e $\alpha_{2}$ pode ser interpretada como um tradeoff entre viés e variância. 
Como mostrado em Ledoit e Wolf (2004), para o caso em que função de perda é quadrática e a distância entre a matriz estimada e a populacional é dada pela norma de Frobenius, podemos encontrar os valores de $\alpha_{1}$ e $\alpha_{2}$ a partir de quatro escalares

$$
\begin{gathered}
\mu=\langle\Sigma, I\rangle \\
\alpha^{2}=\|\Sigma-\mu I\|^{2} \\
\beta^{2}=E\left[\|S-\Sigma\|^{2}\right] \\
\delta^{2}=\alpha^{2}+\beta^{2}=E\left[\|S-\mu I\|^{2}\right]
\end{gathered}
$$

em I é a matriz identidade.

A solução da Equação 1.9 pode ser escrita como

$$
\Sigma^{*}=\frac{\beta^{2}}{\delta^{2}} \mu I+\frac{\alpha^{2}}{\delta^{2}} S
$$

Como analisado em Ledoit e Wolf (2004), note que

$$
\mu=\frac{1}{p} \sum_{i=1}^{p} \lambda_{i}=E\left[\frac{1}{p} \sum_{i=1}^{p} l_{i}\right]
$$

em que $\lambda_{i}$ representa os autovalores de $\Sigma$ e $l_{i}$ representa os autovalores de S. Note também que

$$
\frac{1}{p} E\left[\sum_{i=1}^{p}\left(l_{i}-\mu\right)^{2}\right]=\frac{1}{p} \sum_{i=1}^{p}\left(\lambda_{i}-\mu\right)^{2}+E\left[\|S-\Sigma\|^{2}\right]
$$

A Equação 1.16 indica que os autovalores amostrais são mais dispersos em torno de $\mu$ do que os verdadeiros autovalores e que essa dispersão é igual a $E\left[\|S-\Sigma\|^{2}\right]$. Portanto, a determinação de $\Sigma^{*}$ também leva os autovalores a serem pressionados para valores mais centrais.

No entanto, as Equações 1.14 e 1.16 requerem o conhecimento da verdadeira matriz de covariância $\Sigma$, e essa matriz não é observada. Porém, assintoticamente, existe um estimador consistente para $\mu, \alpha^{2}, \beta^{2}$ e $\delta^{2}$. Portanto, também existe, assintoticamente, um estimador para $\Sigma^{*}$.

Ledoit e Wolf (2004) apresenta o estimador autêntico sobre general asymptotics. Dessa forma, é permitido que o número de variáveis n cresça de acordo com $\mathrm{T}$, sendo indicado como $n_{T}$, mas de forma que $\frac{n_{T}}{T} \leq K_{1}$, em que $K_{1}$ é uma constante que independe de T. Assim como em Ledoit e Wolf (2004), o subscrito T será acrescentado a notação dessa seção para indicar que o resultados são válidos assintoticamente. 
Sejam $m_{T}$ e $d_{T}$ tais que

$$
\begin{gathered}
m_{T}=\left\langle S_{T}, I_{T}\right\rangle \\
d_{T}^{2}=\left\|S_{T}-m_{T} I_{T}\right\|^{2}
\end{gathered}
$$

$m_{T}$ e $d_{T}$ são estimadores consistente para $\mu_{T}$ e $\delta_{T}$.

Considere que

$$
\begin{gathered}
b_{T}^{2}=\min \left(d_{T}^{2}, \bar{b}_{T}^{2}\right) \\
\bar{b}_{T}^{2}=\frac{1}{T^{2}} \sum_{k=1}^{T}\left\|X_{k} X_{k}^{\prime}-S_{T}\right\|^{2}
\end{gathered}
$$

em que $X_{k}$, se refere a k-ésima linha da matriz de dados X. $b_{T}^{2}$ é um estimador consistente para $\beta_{T}^{2}$. Por fim, defina $a_{T}^{2}$ como

$$
a_{T}^{2}=d_{T}^{2}+b_{T}^{2}
$$

$a_{T}^{2}$ é um estimador consistente para $\alpha_{T}^{2}$.

Uma que vez temos estimadores consistentes para os escalares $\mu_{T}, \alpha_{T}^{2}, \beta_{T}^{2}$ e $\delta_{T}^{2}$, temos um estimador consistente para $\Sigma^{*}$ dado por

$$
\hat{\Sigma}^{*}=\frac{b_{T}^{2}}{d_{T}^{2}} m_{T} I_{T}+\frac{a_{T}^{2}}{d_{T}^{2}} S_{T}
$$

Note que esse estimador não depende da verdadeira matriz de covariância $\Sigma$. Portanto, é um estimador genuíno que pode ser utilizado nas aplicações empíricas. 



\section{COMPONENTES PRINCIPAIS E ESPARSIDADE}

\subsection{Análise de componentes principais}

Como descrito em Jolliffe (1986), a PCA é um método estatístico capaz de transformar um conjunto de variáveis interrelacionadas em um novo conjunto de variáveis sintéticas não correlacionadas chamadas de componentes principais.

Como notado por Jolliffe (1986), a PCA pode ser utilizada para obter uma grande redução de dimensionalidade. Segundo Johnson, Wichern et al. (2002), é necessário considerar todas as componentes principais para recuperar a estrutura de variância - covariância presente nos dados originais. Porém, as componentes principais capturam a variabilidade de forma sequencial. Portanto, ao permitir que uma pequena parte da variação presente no sistema original seja descartada, é possível obter uma grande redução na dimensão do problema.

Similarmente a Zou, Hastie e Tibshirani (2006), considere a matriz de dados X. A decomposição em valores singulares de Xé tal que

$$
X=U D V^{\prime}
$$

em que U e V são matrizes ortogonais e D é uma matriz diagonal contendo os valores singulares. $Z=U D$ são as componentes principais e as colunas de $\mathrm{V}$ são os carregamentos (loadings) correspondentes a cada uma das componentes principais.

Alternativamente, como apresentado em Jolliffe (1986), podemos obter Z e V por meio de uma decomposição autovalor-autovetor da matriz de covariância. Defina $\Gamma_{x}=E\left(X^{\prime} X\right)$. Como $\Gamma_{x}$ é uma matriz de covariância, simétrica e positiva definida, ela pode ser decomposta em

$$
\Gamma_{x}=P D P^{\prime}
$$

A matriz $\mathrm{P}$ contêm os autovetores de $\Gamma_{x}$ nas suas colunas. D é uma matriz diagonal, contendo os autovalores de $\Gamma_{x}$, ordenada de forma que $\lambda_{1} \geq \lambda_{2} \geq \cdots \geq \lambda_{n}>0$. O produto das matrizes $\mathrm{X}$ e $\mathrm{P}$ determina as componentes principias, enquanto $\mathrm{P}$ ' é a matriz de carregamento das componentes principais.

\subsection{Análise de componentes principais esparsas}

De acordo com Zou, Hastie e Tibshirani (2006), PCA possuí duas vantagens. Ela captura a variabilidade dos dados de forma sequencial e as componentes principais não são correlacionadas entre si. No entanto, eles argumentam que a PCA possui a desvantagem de 
ser uma combinação linear de todas as variáveis na base de dados. Zou, Hastie e Tibshirani (2006) introduz a SPCA para mitigar essa desvantagem. A SPCA é similar a PCA, mas permite que a matriz de carregamento seja esparsa.

Inicialmente, Zou, Hastie e Tibshirani (2006) conecta PCA a um problema de regressão. Dado que cada componente principal é uma combinação linear de todas as variáveis da base de dados, os carregamentos das componentes principais podem ser recuperados ao regredir cada componente principal em todas as variáveis da base de dados. Ao considerar uma regressão com penalidade, como o LASSO, ao invés de de uma regressão linear, é possível obter uma matriz de carregamento esparsa.

Zou, Hastie e Tibshirani (2006) propõe um algoritmo para a SPCA baseado nos seguintes passos

1. Escolha $\mathrm{r}$ componentes principais e faça com que $A=\left[\alpha_{1}, \alpha_{2}, \ldots, \alpha_{r}\right]$ seja igual a V[,1:r $]$. Inicialmente, considere que $B=\left[\beta_{1}, \beta_{2}, \ldots, \beta_{r}\right]$ seja igual a $\mathrm{A}$.

2. Resolva o problema do LASSO ou (Elastic Net) para cada $j=1,2, \ldots, r$ obtendo novo valores $\beta_{j}$.

3. Calcule a SVD de $X^{\prime} X B$ obtendo novas matrizes U, D e V. Atualize A de modo que $A=U V^{\prime}$.

4. Repita os passos 2 e 3 até convergência.

5. Padronização: $\hat{V}_{j}=\frac{\beta_{j}}{\left\|\beta_{j}\right\|}$ para cada $j=1,2, \ldots, r$.

Na segunda etapa do algoritmo da SPCA, é necessário estimar uma regressão com penalidade. Os resultados dessas regressões dependem da escolha de um parâmetro de penalização. Wang, Li e Leng (2009) sugere generalizar o critério de informação Bayesiano (Bayesian information criterion - BIC) para uma forma mais geral adequada a determinação desse parâmetro. Defina

$$
I C_{m}=\underset{m}{\arg \min } \ln V+m \times \frac{\ln (n T)}{n T} \times C_{n}
$$

$V(\cdot)$ é a variância residual média e $\frac{\ln n T}{n T} C_{n}$ é uma função de penalização com $C_{n}>0$. Note que no caso dos modelos fatoriais $\mathrm{V}$ depende dos fatores $\hat{F}$ e da matriz de carregamento $\hat{\Lambda}$, enquanto m indica o número de entradas que não são zero na matriz de carregamento.

De acordo com Wang, Li e Leng (2009), $C_{n}=\ln \ln (n T)$ leva a bons resultados em amostras finitas. Enquanto Kristensen (2017) sugere o uso de $C_{n}=1$. Nós consideramos ambos os critérios na nossa aplicação empírica. 


\section{MODELOS FATORIAIS}

Modelos fatoriais têm se destacado na literatura de previsão quando consideramos grandes bases de dados. De acordo com Eklund e Kapetanios (2008), tais modelos são caracterizados por

$$
Y_{t}=\lambda(L) f_{t}+e_{t}
$$

$Y_{t}$ é um vetor de variáveis observadas, $f_{t}$ é um vetor com q fatores latentes, $\lambda(L)$ é uma matriz de defasagens polinomiais e $e_{t}$ é um componente idiossincrático que pode ser serialmente correlacionado.

\subsection{Modelo de fatores dinâmicos}

Como discutido em Stock e Watson (2009), o DFM é um dos modelos mais importantes para realizar previsões macroeconômicas. Esse modelo pode ser representado em sua forma estática ou dinâmica.

A forma dinâmica é representada pelo seguinte conjunto de equações

$$
\begin{gathered}
Y_{t}=\lambda(L) f_{t}+e_{t} \\
f_{t}=\psi(L) f_{t-1}+\eta_{t} \\
e_{i t}=\delta_{i}(L) e_{i t-1}+v_{i t}
\end{gathered}
$$

$\psi(L)$ e $\delta(L)$ são matrizes de defasagem polinomiais. $\eta_{t}$ e $v_{i t}$ são componentes idiossincráticos. Assuma que as inovações nos fatores não são correlacionadas com os componentes idiossincráticos.

A forma dinâmica representa a dependência de $Y_{t}$ em relação aos fatores (possivelmente defasados) de forma explícita. Uma representação é apresentada na versão estática do DFM sendo caracterizada por

$$
\begin{gathered}
Y_{t}=\Lambda F_{t}+e_{t} \\
F_{t}=\Phi(L) F_{t-1}+G \eta_{t} \\
G=\left[I_{q} 0\right]^{\prime}
\end{gathered}
$$

Essa representação considera que existem $\mathrm{r}$ fatores estáticos $F_{t}$ ao invés de q fatores dinâmicos. Note que se $r=q$, então $G=I$. A Equação 3.4 indica que $F_{t}$ possui apenas um efeito contemporâneo em $Y_{t}$, diferentemente da Equação 3.1. 
De acordo com Stock e Watson (2016), o DFM em suas formas dinâmicas e estáticas são equivalentes. É possível alterar entre essas representações por meio das seguintes equações

$$
\begin{gathered}
\Lambda=\left(\lambda_{0}, \lambda_{1}, \ldots, \lambda_{p}\right) \\
F_{t}=\left(f_{t}^{\prime}, f_{t-1}^{\prime}, \ldots, f_{t-p}^{\prime}\right)^{\prime}
\end{gathered}
$$

Tanto na versão estática quanto na dinâmica, podemos identificar uma componente comum, $\chi_{t}=\lambda(L) f_{t}=\Lambda F_{t}$, representando comovimentos e uma componente idiossincrática, $e_{t}$, capaz de capturar características individuais.

\subsection{Hipóteses de identificação}

Nessa seção temos uma breve revisão das principais hipóteses apresentadas em Stock e Watson (2002). Uma alternativa é o conjunto de hipóteses usadas em Fan, Wang e Zhong (2019). Considere as seguintes hipóteses:

$H_{1}: \frac{\Lambda^{\prime} \Lambda}{n} \rightarrow I_{r}$

$H_{2}: E\left(F_{t} F_{t}^{\prime}\right)=\Gamma_{F}$ em que $\Gamma_{F}$ é diagonal com $\sigma_{i i}>\sigma_{j j}>0$ for $i<j$.

$H_{3}: T^{-1} \sum_{t} F_{t} F_{t}^{\prime} \rightarrow \Gamma_{F}$

$H_{4}: E\left(\frac{e_{t}^{\prime} e_{t+u}}{n}\right)=\gamma_{n, t}$ e $\lim _{n \rightarrow \infty} \sup _{t} \sum_{u=-\infty}^{\infty}\left|\gamma_{n, t}\right|<\infty$

$H_{5}: E\left(e_{i t} e_{j t}\right)=\tau_{i j, t}$ e $\lim _{n \rightarrow \infty} \sup _{t} n^{-1} \sum_{i=1}^{n} \sum_{j=1}^{n}\left|\tau_{i j, t}\right|<\infty$

$H_{1}, H_{2}$ e $H 3$ são usadas para identificar os fatores. Ao considerar que $\frac{\Lambda^{\prime} \Lambda}{n}$ converge para uma matriz positiva definida, exclui-se a possibilidade de que algum fator tenha carregamento igual a zero para alguma variável no painel de dados. Como $\Lambda F_{t}=\Lambda R R^{-1} F_{t}, H_{1}$ é usada para limitar R a uma matriz ortonormal. $H_{2}$ restringe $\mathrm{R}$ a uma matriz diagonal com elementos \pm 1 . $H_{4}$ permite que exista correlação serial no processo $e_{i t}$. $H_{5}$ possibilita que exista correlação na cross-section.

\subsection{Estimação e previsão}

Visto que os fatores são variáveis latentes, é necessário usar algum método estatístico para extrai-los. Nesse trabalho, consideramos métodos como PCA e SPCA. Assim como em Stock e Watson (2002), considere o seguinte problema de mínimos quadrados não lineares

$$
V(\tilde{F}, \tilde{\Lambda})=(n T)^{-1} \sum_{i} \sum_{t}\left(y_{i t}-\tilde{\lambda}_{i} \tilde{F}_{t}\right)
$$


escrito e função de fatores e carregamentos hipotéticos, $\tilde{F}$ e $\tilde{\Lambda}$, respectivamente. Seja $\hat{F}$ e $\hat{\Lambda}$ os valores que minimizem a Equação 3.9.

Ao concentrar em $\hat{F}$, minimizar a Equação 3.9 é equivalente a

$$
\max \operatorname{tr}\left[\tilde{\Lambda}^{\prime} Y^{\prime} Y \tilde{\Lambda}\right] \text { s. a. } \frac{\tilde{\Lambda}^{\prime} \tilde{\Lambda}}{n}=I_{r}
$$

Note que o problema representado na Equação 3.10 é o mesmo que o de componentes principais. Portanto, podemos resolve-lo ao fazer que $\hat{F}$ seja igual aos autovetores de $X^{\prime} X$, de modo que $F=\frac{X^{\prime} \hat{\Lambda}}{n}$. Adicionalmente, $\hat{\Lambda}$ é estimado por meio dos autovalores de $X^{\prime} X$. Note que podemos obter a mesma solução considerando a decomposição em valores singulares (SVD).

De acordo com Stock e Watson (2002), PCA fornece um forma consistente de estimar os fatores latentes. Porém, pode ter um desempenho ruim em amostras finitas. Portanto, também consideraremos a extração por SPCA.

Após recuperar os fatores e a matriz de carregamento, precisamos estimar os demais parâmetros. Uma das formas mais simples de estima-los é apresentada em Giannone, Reichlin e Sala (2004) e Forni et al. (2009). Ambos usam PCA para estimar os fatores e a matriz de carregamento e estimam os demais parâmetros via VAR.

Após a estimação, o DFM pode ser usado para realizar previsões das variáveis desejadas iterativamente, como discutido em Clements e Hendry (1998).

\subsection{Número de fatores latentes}

Tanto a PCA quanto SPCA permitem recuperar os fatores latentes. Porem, é necessário determinar o número de fatores que desejamos extrair. De acordo com Fan, Wang e Zhong (2019), podemos determinar a quantidade de fatores ao analisar os autovalores da matriz de covariância amostral ou versões robustas dessa matriz.

Ahn e Horenstein (2013) propõe um estimador para escolher o número de fatores dado por

$$
\hat{r}^{A H}=\underset{i \leq \text { r.max }}{\operatorname{argmax}} \frac{\lambda_{i}}{\lambda_{i+1}}
$$

para um determinado número máximo de fatores determinados a priori r.max, $\hat{r}^{A H}$ é o número estimado de fatores baseado na razão de autovalores consecutivos, $\lambda_{i}$ e $\lambda_{i+1}$, da matriz de covariância dos dados.

Onatski (2010) introduz uma alternativa baseada na diferença entre autovalores con- 
secutivos. Para um número máximo de fatores r.max e para $\delta>0$ defina

$$
\hat{r}^{O}=\max \left\{i \leq \operatorname{r.max}:\left(\lambda_{i}-\lambda_{i+1}\right) \geq \delta\right\}
$$

como o número de fatores estimados pelo critério de Onatski. Em aplicações práticas, o valor de $\delta$ é calibrado por meio de um procedimento descrito em Onatski (2010).

Bai e Ng (2002) propõe um terceiro procedimento para determinar o número de fatores latentes. Defina

$$
I C=\ln (V(r, \hat{F}))+r\left(\frac{n+T}{n T}\right) \ln \left(\frac{n T}{n+T}\right)
$$

$V(r, \hat{F})$ é a variância residual média quando o modelo é estimado com $\mathrm{r}$ fatores, e $\left(\frac{n+T}{n T}\right) \ln \left(\frac{n T}{n+T}\right)$ é uma função de penalização que depende tanto de n quanto de T. De acordo com Bai e Ng (2002), o número de fatores estimados é o valor r que minimiza IC.

\subsection{Comparação de previsões}

Na literatura de previsões macroeconômicas, os modelos costumam ser avaliados por um métrica de erro. Uma das métricas mais populares é o MSFE. Apesar de fornecer informações sobre a qualidade das previsões, o MSFE não indica claramente se diferentes modelos produzem previsões significativamente diferentes.

Uma alternativa capaz de contornar essa limitação é o MCS introduzido por Hansen, Lunde e Nason (2011). O algoritmo do MCS testa sequencialmente a hipótese nula de mesma habilidade preditiva (equal predictive ability - EPA) entre modelos, e baseado em um regra de eliminação, determina os modelos com os melhores desempenhos preditivos.

Tal procedimento permite construir um conjunto que contenha os melhores modelos, em termos preditivos, dado uma determinada função de perda e um nível de confiança. Assim como notado por Bernardi e Catania (2018), o procedimento começa determinando o diferencial de perda entre um número $\mathrm{M}$ de modelos em duas formas diferentes

$$
\begin{gathered}
d_{i j, t}=l_{i, t}-l_{j, t} \\
d_{i, t}=\frac{1}{M-1} \sum_{j} d_{i j, t}
\end{gathered}
$$

$l_{i, t}$ e $l_{j, t}$ são as perdas associadas aos modelos i e j no período t. Duas das funções de perda mais populares são a perda quadrática e a perda absoluta. A primeira calcula a distância entre grandezas elevando a diferença entre elas ao quadrado, já a segunda considera o módulo 
dessa diferença. Portanto, o MCS consegue se beneficiar das informações usadas para o cálculo de métricas como o MSE.

Assim como em Bernardi e Catania (2018), o MCS usa duas estatísticas, $T_{R, M}$ e $T_{\text {max }, M}$, para testar a hipótese nula de EPA entre os modelos.

$$
\begin{gathered}
T_{R, M}=\max \left|t_{i j}\right| \\
T_{\text {max }, M}=\max t_{i}
\end{gathered}
$$

em que $t_{i j}$ e $t_{i}$ são definidos como

$$
\begin{aligned}
t_{i j} & =\frac{\bar{d}_{i j}}{\sqrt{v \hat{a} r\left(\bar{d}_{i j}\right)}} \\
t_{i} & =\frac{\bar{d}_{i}}{\sqrt{v \hat{a} r\left(\bar{d}_{i}\right)}}
\end{aligned}
$$

$\bar{d}_{i j}$ e $\bar{d}_{i}$ são as versões amostrais das Equações 3.14 e 3.15. $v \hat{a} r\left(\bar{d}_{i j}\right)$ e $v \hat{a} r\left(\bar{d}_{i}\right)$ são estimados usando bootstrap.

Se $H_{0}$ não é rejeitado, o algoritmo do MCS é interrompido e todos os M modelos pertencem ao conjunto de modelos com mesma habilidade preditiva. Caso contrário, o algoritmo usa uma regra de eliminação como

$$
e_{\max , M}=\operatorname{argmax} \frac{\bar{d} i}{v \hat{a} r(\bar{d} i)}
$$

para eliminar o modelo com o pior desempenho. Após a eliminação, o procedimento reinicia com M-1 modelos e é repetido até que reste apenas 1 modelo ou até que não seja possível rejeitar $H_{0}$. 



\section{MODELOS CONCORRENTES}

Além do DFM, muitos outros métodos podem ser usados para realizar previsões no contexto de alta dimensão. Porém, é necessário limitar o número de modelos preditivos para comparação. Nesse trabalho, além do DFM, consideramos regressões com penalização e o GDFM. Tais modelos são justificados destacam-se na literatura como mostrado, por exemplo, em Medeiros, Vasconcelos e Freitas (2016), Smeekes e Wijler (2018), Forni et al. (2005) e Barigozzi e Hallin (2020).

Medeiros, Vasconcelos e Freitas (2016) analisa o desempenho preditivo de métodos de alta dimensão como o LASSO, adaLASSO, Elastic Net, bagging e target factors em relação ao $\mathrm{AR}(4)$. Os autores encontram que os métodos de alta dimensão produzem RMSE menores que o benchmark para a maioria das variáveis macroeconômicas previstas. Os autores ressaltam que a melhora na previsão é menos conclusiva para o método target factors.

Adicionalmente, Smeekes e Wijler (2018) apresenta evidências de que modelos de regressão com penalidade são mais robusto a erros de especificações do que modelos fatoriais.

Já Forni et al. (2005) e Barigozzi e Hallin (2020) argumentam que o GDFM é mais flexível que o DFM sendo capaz de capturar toda a dinâmica da estrutura de covariância dos dados. Adicionalmente, Forni et al. (2005) comenta que uma das vantangens do GDFM é o fato dele atribuir peso as variáveis de acordo com a razão sinal-ruído estimada.

Portanto, é razoável compararmos o desempenho preditivo entre as regressões com penalização, GDFM e DFM.

\subsection{Regressões com penalização}

\subsubsection{Regressão Ridge}

A regressão Ridge é um método de seleção de variáveis proposto por Hoerl e Kennard (1970). Esse método baseia-se no OLS, porém penaliza os coeficientes da regressão.

De acordo com Friedman, Hastie e Tibshirani (2001), os coeficientes estimados pela regressão Ridge são dados por

$$
\hat{\beta}^{\text {Ridge }}=\underset{\beta}{\operatorname{argmin}}\left\{\sum_{i=1}^{n}\left(y_{i}-\beta_{0}-\sum_{j=1}^{p} x_{i j} \beta_{j}\right)^{2}+\kappa \sum_{j=1}^{p} \beta_{j}^{2}\right\}
$$

em que $\mathrm{Y}$ representa a variável de interesse, $\mathrm{X}$ indica a matriz de possíveis preditores $\mathrm{e}$ $\hat{\beta}^{\text {Ridge }}$ é a matriz de coeficientes obtidos pela regressão Ridge. 
A Representação 4.1 indica que a regressão Ridge minimiza a soma do quadrado dos resíduos sujeito a uma penalização da norma $\ell_{2}$, dada por $\sum_{j=1}^{p} \beta_{j}^{2}$, e com um parâmetro $\kappa$ que controla a magnitude da penalidade. Note que se $\kappa=0, \hat{\beta}^{\text {Ridge }}=\hat{\beta}^{O L S}$.

Friedman, Hastie e Tibshirani (2001) indica que a regressão Ridge pode ser escrita na forma de um problema de minimização.

$$
\hat{\beta}^{\text {Ridge }}=\underset{\beta}{\operatorname{argmin}} \sum_{i=1}^{n}\left(y_{i}-\beta_{0}-\sum_{j=1}^{p} x_{i j} \beta_{j}\right)^{2} \text { s. a. } \sum_{j=1}^{p} \beta_{j}^{2} \leq t
$$

A Representação 4.2 torna explicita a restrição no tamanho dos coeficientes.

Caso duas variáveis sejam altamente correlacionadas, a imposição da penalização na regressão leva a seleção de uma dessas variáveis para ser incorporada ao modelo, como descrito em Friedman, Hastie e Tibshirani (2001).

\subsubsection{LASSO}

O LASSO é um método de shrinkage proposto por Tibshirani (1996) como uma alternativa a regressão Ridge. Assim como a Ridge, o LASSO penaliza os coeficientes da regressão linear. No entanto, os métodos diferem no tipo de penalização. Enquanto o Ridge penaliza pela norma $\ell_{2}$, a penalização do LASSO é baseada na norma $\ell_{1}$.

Os coeficientes obtidos pelo LASSO são representados por

$$
\hat{\beta}^{L A S S O}=\underset{\beta}{\arg \min }\left\{\sum_{i=1}^{n}\left(y_{i}-\beta_{0}-\sum_{j=1}^{p} x_{i j} \beta_{j}\right)^{2}+\kappa \sum_{j=1}^{p}\left|\beta_{j}\right|\right\}
$$

em que $\mathrm{Y}$ representa a variável de interesse, $\mathrm{X}$ indica a matriz de possíveis preditores e $\hat{\beta}^{L A S S O}$ é a matriz de coeficientes obtidos pelo LASSO.

Similarmente a regressão Ridge, o LASSO pode ser obtido por meio de um problema de minimização:

$$
\hat{\beta}^{\text {LASSO }}=\underset{\beta}{\operatorname{argmin}} \sum_{i=1}^{n}\left(y_{i}-\beta_{0}-\sum_{j=1}^{p} x_{i j} \beta_{j}\right)^{2} \text { s. a. } \sum_{j=1}^{p}\left|\beta_{j}\right| \leq t
$$

Assim como na Equação 4.2, essa representação torna explicita a restrição imposta aos coeficientes do modelo. Note que se $\kappa$ é igual a zero, A Equação 4.3 é reduzida a solução de mínimos quadrados. Já no caso em que $\kappa$ tende ao infinito, todos os coeficientes são reduzidos a zero.

Para valores suficientemente grandes, o LASSO é capaz de produzir soluções esparsas e realizar seleção de variáveis. Devido a esparsidade, esse método produz soluções mais 
parcimoniosas do que as obtidas por métodos tradicionais como o de mínimos quadrados ordinários. Além disso, Tibshirani, Wainwright e Hastie (2015) indica que ao usar o LASSO é possível beneficiar-se do tradeoff entre viés e variância para obter estimativas mais precisas em amostras finitas.

A determinação do parâmetro de penalização é um dos aspectos críticos na estimação do LASSO. Tipicamente, esse parâmetro é determinado por validação cruzada ou por algum critério de informação.

No caso de séries temporais, a aplicação de validação cruzada pode ser problemática devido a dependência temporal. Adicionalmente, critérios de informação tem a vantagem de que é necessário estimar a trajetória de regularização uma única vez.

\subsubsection{Elastic Net}

Zou e Hastie (2005) indica três limitações do LASSO

1. Se o número de potenciais preditores, representado por $\mathrm{p}$, é maior que o número de observações, representado por n, o LASSO é capaz de selecionar no máximo n preditores.

2. Se um grupo de variáveis possui um correlação par a par muito alta, o LASSO tende a selecionar apenas uma dessa variáveis.

3. No caso $n>p$, se os preditores possuem correlação alta, a regressão Ridge, geralmente, possui um melhor desempenho preditivo do que o LASSO.

Para mitigar essas limitações, Zou e Hastie (2005) propõe uma regressão com penalidade, nomeada Elastic Net, capaz de combinar as penalizações da regressão Ridge e do LASSO.

De acordo com Zou e Hastie (2005), os coeficientes obtidos pelo Naive Elastic Net são representados por

$$
\hat{\beta}^{E N}=\underset{\beta}{\operatorname{argmin}}\left\{\sum_{i=1}^{n}\left(y_{i}-\beta_{0}-\sum_{j=1}^{p} x_{i j} \beta_{j}\right)^{2}+\kappa_{1} \sum_{j=1}^{p}\left|\beta_{j}\right|+\kappa_{2} \sum_{j=1}^{p} \beta_{j}^{2}\right\}
$$

Zou e Hastie (2005) mostra que se considerarmos $\alpha=\frac{\kappa_{2}}{\kappa_{1}+\kappa_{2}}$, o coeficiente obtido pelo Naive Elastic Net pode ser representado por

$$
\hat{\beta}^{E N}=\underset{\beta}{\operatorname{argmin}} \sum_{i=1}^{n}\left(y_{i}-\beta_{0}-\sum_{j=1}^{p} x_{i j} \beta_{j}\right)^{2} \text { s. a. }(1-\alpha) \sum_{j=1}^{p}\left|\beta_{j}\right|+\alpha \sum_{j=1}^{p} \beta_{j}^{2} \leq t
$$


No caso em $\alpha=1$, o problema é reduzido ao caso da regressão Ridge. Se $\alpha=0$, temos o problema é simplificado para o caso do LASSO.

Assim como a regressão Ridge e o LASSO, o Elastic Net pode ser usado para obter previsões mais precisas por meio do tradeoff entre viés e variância.

\subsection{4 adaLASSO}

De acordo com Zou, Hastie e Tibshirani (2006) e reforçado por Medeiros, Vasconcelos e Freitas (2016), o LASSO não possui a propriedade do oráculo e tem seu desempenho prejudicado quando número de variáveis cresce. Como o intuito de combater essas desvantagens, Zou, Hastie e Tibshirani (2006) introduz o adaLASSO.

Os coeficientes obtidos pelo adaLASSO são representados por

$$
\hat{\beta}^{\text {adaLASSO }}=\underset{\beta}{\operatorname{argmin}}\left\{\sum_{i=1}^{n}\left(y_{i}-\beta_{0}-\sum_{j=1}^{p} x_{i j} \beta_{j}\right)^{2}+\kappa \sum_{j=1}^{p} w_{j}\left|\beta_{j}\right|\right\}
$$

em que $w_{j}=\left|\hat{\beta}_{j}^{*}\right|^{-1}$ com $\beta_{j}^{*}$ estimado previamente. Como indicado por Medeiros, Vasconcelos e Freitas (2016), $\beta_{j}^{*}$ pode ser estimado usando, por exemplo, LASSO ou Elastic Net. A partir dessa modificação, o adaLASSO obtém a propriedade do oráculo.

\subsection{Generalized Dynamic Factor Model}

De acordo com Forni e Lippi (2011), o GDFM é baseado na seguinte decomposição

$$
y_{i t}=\chi_{i t}+\xi_{i t}=b_{i 1}(L) u_{1 t}+\cdots+b_{i q}(L) u_{q t}+\xi_{i t} \operatorname{com} i \in N, t \in Z
$$

em que $\xi_{i t}$ é a componente idiossincrática, $\chi_{i t}$ a componente comum, $b_{i f} \operatorname{com} f=1, \ldots, q$ são os filtros e $u_{t}$ é um vetor q-dimensional de ruídos branco ortonormais que representam os choques comuns. Forni e Lippi (2011) destaca duas hipóteses do modelo:

H1) $u_{t}$ é ortogonal a $\xi_{i t-k}$ para todo $i \in N$ e $k \in Z$.

H2) As covariâncias cruzadas entre os $\xi$ s é fraca.

Forni e Lippi (2011) indica que a segunda hipótese é muito mais fraca que exigir que $\xi$ s tenham covariância cruzada igual a 0 e, por esse motivo, chama o modelo de GDFM.

Como discutido em Forni et al. (2000), o GDFM pode ser estimado pelo método de componentes principais dinâmicas introduzido por Brillinger (1981). No entanto, essa estimação não é adequada para o contexto de previsões.

Porém, Forni et al. (2005) apresenta um procedimento adequado para previsões baseado em Forni et al. (2000). 
Forni et al. (2005) indica que é possível aproximar o espaço dos fatores por meio de combinações lineares dos dados observados. Tal aproximação é possível porque se a dimensão da cross-section tende a infinito, a componente idiossincrática tende a 0 devido a $\mathrm{H} 2)$.

A previsão da componente comum é obtida ao projetarmos os valores futuros de $\chi$ no espaço gerado pelo fatores como na Equação 3.2 de Forni et al. (2005). Já a componente idiossincrática pode ser modelada usando técnicas univariadas.

Note ainda que é necessário determinar o número de choques comuns no modelo. Para isso, podemos usar o procedimento apresentado em Hallin, Liska et al. (2007). Nesse procedimento o número de choques comuns é determinado por um critério de informação baseado no número de autovalores divergentes da matriz de densidade espectral. 



\section{APLICAÇÃO EMPÍRICA}

\subsection{Base de dados}

Nesse trabalho será utilizada a base de variáveis macroeconômicas apresentada em Stock e Watson (2009). Essa é uma base de dados conhecida na literatura sendo utilizada em Groen e Kapetanios (2016), Aiolfi, Capistrán e Timmermann (2010) e Castle, Clements e Hendry (2013) e, após atualizações, em Stock e Watson (2012) e Koop (2013).

Tal base de dados consiste, originalmente, em 144 séries temporais representando variáveis macroeconômicas medidas trimestralmente para a economia americana de 1959:1 até 2006:4. Similarmente a Stock e Watson (2012), essa variáveis podem ser classificadas em 13 grupos, como apresentado na tabela abaixo.

\begin{tabular}{ccc}
\hline Grupo & Descrição & Número de Séries \\
\hline 1 & Componentes do PIB & 16 \\
2 & Produção industrial & 14 \\
3 & Emprego & 20 \\
4 & Taxa de desemprego & 7 \\
5 & Habitação & 6 \\
6 & Inventário & 6 \\
7 & Preços & 37 \\
8 & Salários & 6 \\
9 & Taxa de juros & 13 \\
10 & Moeda & 8 \\
11 & Taxa de câmbio & 5 \\
12 & Mercado de ações & 5 \\
13 & Expectativas & 1 \\
\hline
\end{tabular}

Tabela 5.1 - Classificação das séries temporais contidas na base de dados apresentada em Stock e Watson (2009).

Como discutido em Stock e Watson (2009), as realizações de 1959:1 e 1959:2 são usadas no cálculo da primeira e segunda diferença para obter estacionariedade nas séries. A primeira diferença em log é usada para variáveis em quantidades reais, a primeira diferença é usada para taxas de juros nominais e a segunda diferença em log é usada para as séries de preço. Após esse tratamento, ficam disponíveis os dados de 1959:3 até 2006:4, totalizando T = 190 realizações no tempo.

Além disso, Stock e Watson (2009) remove 35 séries por representarem medidas consideradas muito agregadas pelos autores. Essas séries temporais podem ser reconstruídas com as informações presentes nas 109 variáveis restantes e não fornecem novas informações para a construção dos fatores. Por exemplo, a variável de número de empregados total (CES002) é 
removida, porém ela pode ser reconstruída a partir do número de empregados em cada um dos setores. Uma descrição detalhada das séries originalmente disponibilizadas, bem como o tipo de transformação usada para obter estacionariedade e um indicador se a série foi usada na construção dos fatores é disponibilizado em Stock e Watson (2009).

A Tabela 5.2 mostra a quantidade de séries temporais presentes em cada grupo após a remoção das 35 séries.

\begin{tabular}{ccc}
\hline Grupo & Descrição & Número de Séries \\
\hline 1 & Componentes do PIB & 10 \\
2 & Produção industrial & 9 \\
3 & Emprego & 16 \\
4 & Taxa de desemprego & 7 \\
5 & Habitação & 4 \\
6 & Inventário & 6 \\
7 & Preços & 24 \\
8 & Salários & 5 \\
9 & Taxa de juros & 9 \\
10 & Moeda & 8 \\
11 & Taxa de câmbio & 5 \\
12 & Mercado de ações & 5 \\
13 & Expectativas & 1 \\
\hline
\end{tabular}

Tabela 5.2 - Número de séries temporais presentes em cada um dos grupos após a remoção das 35 séries realizadas em Stock e Watson (2009).

As últimas 20 realizações de cada série temporal são removidas para serem usadas na avaliação das previsões. Em sequencia, as séries são padronizadas.

\subsection{Estimação}

Nesse trabalho, consideramos a extração dos fatores do DFM por PCA e por SPCA. Em cada um dos casos é necessário determinar a matriz que modelará a dependência entre as séries e o número de fatores que serão incluídos na análise. Nessa aplicação empírica, consideraremos que existem no máximo 7 fatores latentes. Adicionalmente, no caso do SPCA, é necessário determinar a penalização, ou o número de variáveis não nulas, usadas para recuperar os fatores. Após isso, podemos recuperar os fatores latentes e, em sequência, determinar os demais parâmetros do DFM por meio do VAR. Para estimar esses modelos, consideramos uma janela móvel contendo 170 observações.

Após a estimação do DFM, o modelo pode ser usado para realizar previsões. Nessa aplicação empírica desejamos prever as versões estacionárias das séries do PIB real (GDP251), índice de produção industrial (IPS10), taxa de desemprego para as pessoas acima de 16 anos 
(LHUR), índice de preços do consumidor (CPIAUCSL) e índice S\&P 500 (FSPCOM) como descrito na Tabela 5.3.

\begin{tabular}{cc}
\hline Sigla & Descrição \\
\hline GDP251 & PIB Real \\
IPS10 & Índice de produção industrial \\
LHUR & Taxa de desemprego para pessoas acima de 16 anos \\
CPIAUCSL & Índice de preços ao consumidor \\
FSPCOM & Índice S\&P 500 \\
\hline
\end{tabular}

Tabela 5.3 - Descrição das séries a serem previstas e sua sigla correspondente.

Neste trabalho, consideramos previsões para os próximos 1, 2, 4, 6 e 8 trimestres. Esses horizontes de previsão foram escolhidos de modo a capturar comportamentos de curto e médio prazo para as variáveis de interesse. Por fim, avaliamos o desempenho das previsões por meio do MSFE e pelo MCS.

Inicialmente, separamos os modelos de acordo com a matriz usada para modelar a dependência entre as séries. Após apresentar os modelos sugeridos em cada um dos casos, consideramos alguns casos de interesse para realizar as previsões. Por fim, comparamos o desempenho preditivo entre os modelos fatoriais e em relação aos modelos baseados em regressões com penalizações e ao generalized dynamic factor model.

\subsubsection{Matriz de covariância amostral}

Inicialmente, consideramos que a dependência entre as séries é modelada pelo equivalente amostral da matriz de covariância. A partir da matriz de covariância amostral, calculamos em cada janela os critérios de Ahn e Horenstein (2013), Onatski (2010) e Bai e Ng (2002). Os resultados encontrados são sumarizados pela Figura 5.1.

Os critérios de Ahn e Horenstein e de Onatski sugerem um único fator latente. Já o critério de Bai e Ng, assim como observado em Stock e Watson (2009), aumenta de 3 para 4 fatores latentes ao longo das janelas. Ao invés de impor que existe o mesmo número de fatores em todos os períodos, permitiremos que o DFM seja estimado com um número diferente de fatores para janelas distintas.

Note que é necessário estimar os fatores e matriz de carregamento para a utilização do critério de Bai e Ng. Logo, o método de extração dos fatores poderá alterar o desempenho do modelo. Bai e Ng (2002) consideram que esse critério de informação possui um bom desempenho em amostras finitas para o caso da extração dos fatores por PCA. Portanto, esse critério será utilizado apenas para avaliar o DFM com fatores extraídos por PCA.

Adicionalmente, usamos os critérios propostos por Kristensen (2017) e Wang, Li e Leng (2009) para determinar o número de variáveis não nulas no SPCA para cada janela. Os 


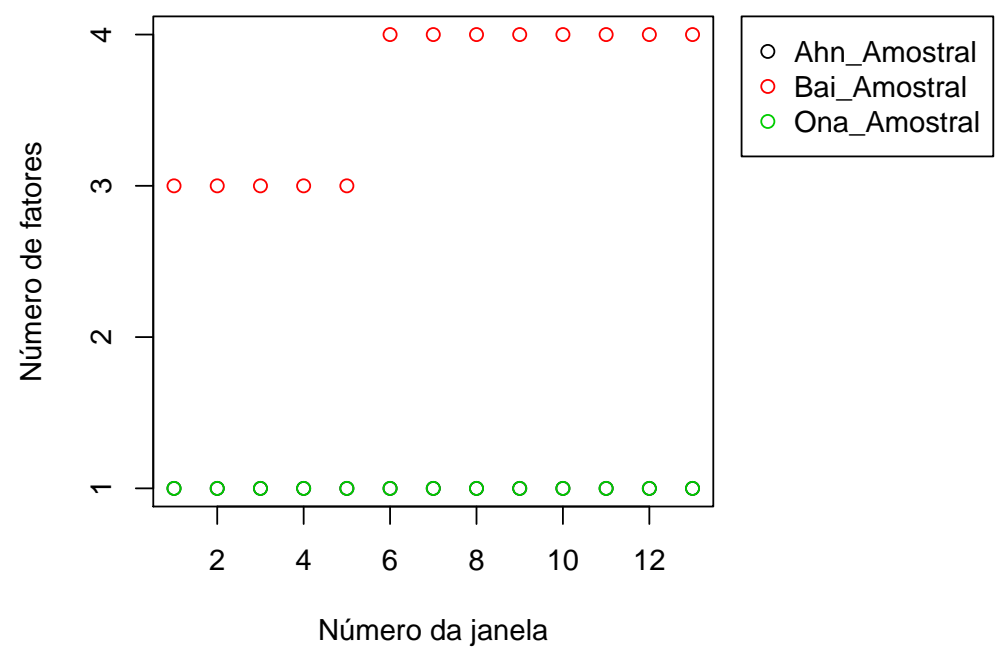

Figura 5.1 - Número de fatores sugeridos pelos critérios apresentados em Ahn e Horenstein (2013), Bai e Ng (2002) e Onatski (2010) quando modelamos a dependência entre a séries por meio da matriz de covariância amostral

resultados desses critérios são apresentados na Figura 5.2.

Esse resultado mostra que o critério de penalização proposto por Wang, Li e Leng leva a uma penalização mais severa que a baseada no critério de Kristensen.

Adicionalmente, consideramos o caso em que ocorre um penalização intermediária as propostas pelos dois critérios anteriores. Consideramos o caso em que 61 variáveis são usadas na construção dos fatores em todas as janelas. Tal valor corresponde a média das medianas propostas pelos critérios de Kristensen e Wang e seus coautores. A partir desses critérios são propostos os modelos apresentados na Tabela 5.4.

\begin{tabular}{cccc}
\hline ID do Modelo & Método de extração & No de fatores & Penalização \\
\hline M01PC & PCA & $1 \mathrm{~F}$ & - \\
M02PC & PCA & BaiNg & - \\
M01SPC & SPCA & $1 \mathrm{~F}$ & Kristensen \\
M02SPC & SPCA & $1 \mathrm{~F}$ & Wang \\
M03SPC & SPCA & $1 \mathrm{~F}$ & Fixa(61) \\
\hline
\end{tabular}

Tabela 5.4 - Modelos usados para previsão quando consideramos que a dependência entre séries é modelada pela matriz de covariância amostral 


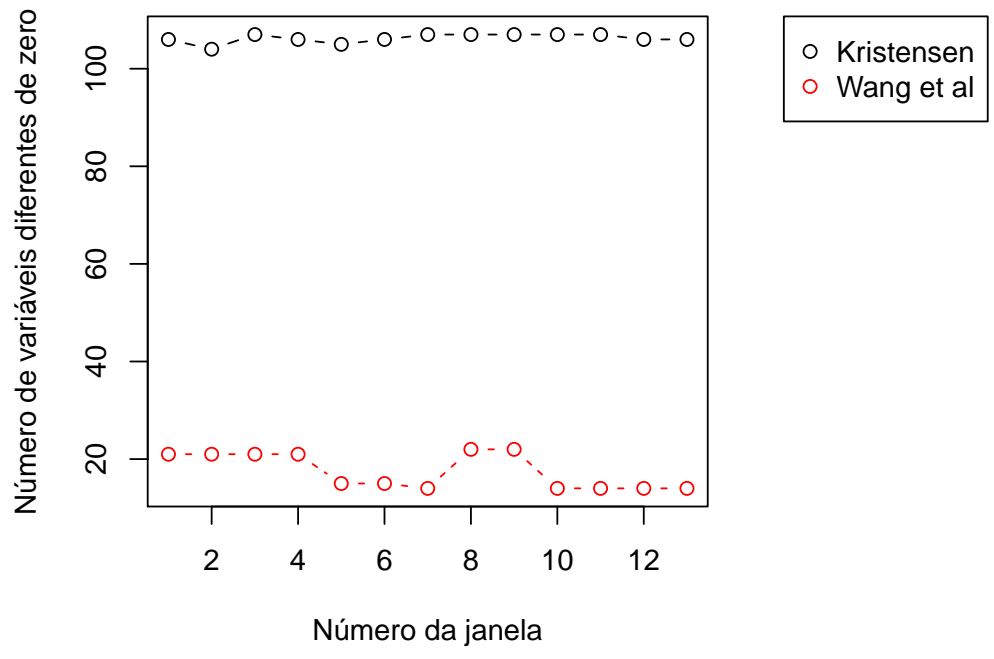

Figura 5.2 - Número de variáveis não nulas sugeridas pelos critérios de Kristensen (2017) e Wang, Li e Leng (2009) quando modelamos a dependência entre a séries por meio da matriz de covariância amostral

\subsubsection{LRCM de Andrews}

Como discutido na Seção 1.1, o uso da matriz de covariância amostral pode ser inadequado para a aplicação empírica. Portanto, consideraremos o uso da LRCM proposta como o da classe proposta por Andrews (1991). Tal matriz pode ser calculada no R por meio do pacote sandwich introduzido por Zeileis (2004).

Ao substituir a matriz de covariância amostral pela LCRM, é necessário recalcular o número de fatores latentes por meio dos critérios de Ahn e Horenstein, Bai e Ng e Onatski. Os resultados desses novos cálculos são apresentados na Figura 5.3

Ao considerar a LRCM de Andrews, todos os critérios apontam, para a maioria das janelas, a existência de um único fator. Portanto, consideramos que existe apenas 1 fator latente.

Novamente, usamos os critérios propostos por Kristensen (2017) e Wang, Li e Leng (2009) para determinar o número de variáveis não nulas no SPCA. Os resultados desses critérios são apresentados na Figura 5.4.

Note que as penalizações para o caso em que consideramos a LRCM é mais severa que no caso em que usamos a covariância amostral.

Similarmente ao caso anterior, consideramos também o caso em que a extração é realizada por SPCA e com um número fixo de variáveis na composição dos fatores. Nesse caso, 


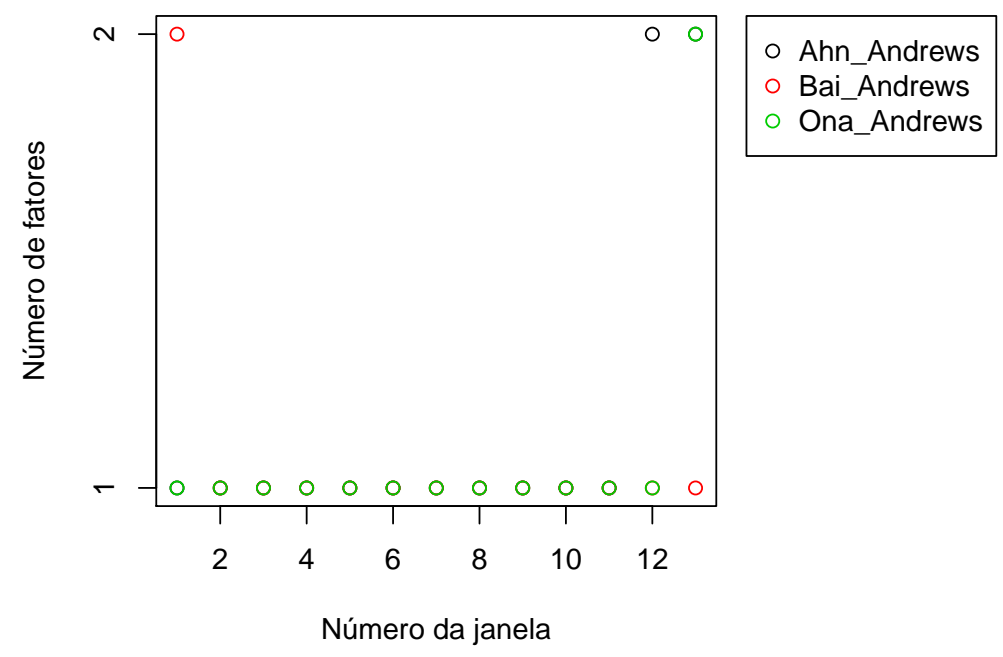

Figura 5.3 - Número de fatores sugeridos pelos critérios apresentados em Ahn e Horenstein (2013), Bai e Ng (2002) e Onatski (2010) quando modelamos a dependência entre a séries por meio da matriz de covariância de longo prazo proposta em Andrews (1991)

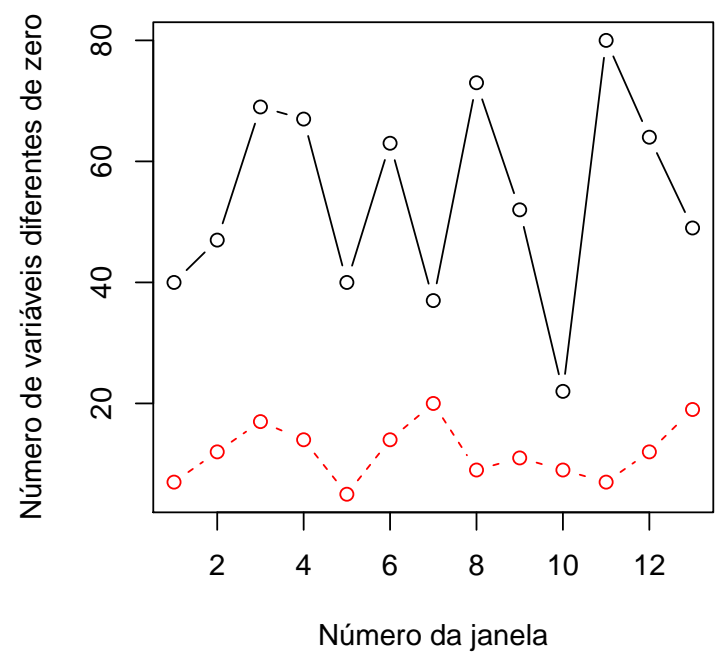

Kristensen

Wang et al

Figura 5.4 - Número de variáveis não nulas sugeridas pelos critérios de Kristensen (2017) e Wang, Li e Leng (2009) quando modelamos a dependência entre a séries por meio da LRCM proposta por Andrews (1991). 
consideramos 32 variáveis na composição de cada fator. Baseado nesses critérios, estimamos os modelos apresentados na Tabela 5.5.

\begin{tabular}{cccc}
\hline ID do Modelo & Método de extração & No de fatores & Penalização \\
\hline M03PC & PCA & $1 \mathrm{~F}$ & - \\
M04SPC & SPCA & $1 F$ & Kristensen \\
M05SPC & SPCA & $1 F$ & Wang \\
M06SPC & SPCA & $1 F$ & Fixa(32) \\
\hline
\end{tabular}

Tabela 5.5 - Modelos usados para previsão quando consideramos que a dependência entre séries é modelada pela LRCM proposta por Andrews (1991).

\subsubsection{LRCM de Kiefer e Vogelsang}

Devido a problema de robustez à erros de medidas na LRCM de Andrews (1991), apontado por Müller (2007), analisaremos o efeito de substituir a matriz de covariância amostral pela LRCM de Kiefer e Vogelsang (2002).

Após a substituição devemos calcular, novamente, os critérios de Ahn e Horenstein, Bain e Ng, e Onatski para determinar o número de fatores latente na aplicação. Esse valores são apresentados na Figura 5.5. Adicionalmente, devemos recalcular a penalização do método SPCA. Também consideramos o caso em que os fatores são constituídos por 41 variáveis em todas as janelas. As penalidades sugeridas são apresentadas na Tabela 5.6.

Como mostrado na Figura 5.5, os critérios de Ahn e Horenstein e de Onatski sugerem um único fator em todos os casos, diferentemente de quando é usada a LRCM de Andrews (1991).

Esses critério sugerem a estimação de quatro modelos, conforme apresentado na Tabela 5.6.

\begin{tabular}{cccc}
\hline ID do Modelo & Método de extração & No de fatores & Penalização \\
\hline M04PC & PCA & $1 \mathrm{~F}$ & - \\
M05PC & PCA & $4 \mathrm{~F}$ & - \\
M07SPC & SPCA & $1 \mathrm{~F}$ & Kristensen \\
M08SPC & SPCA & $1 \mathrm{~F}$ & Wang \\
M09SPC & SPCA & $1 \mathrm{~F}$ & Fixa(41) \\
\hline
\end{tabular}

Tabela 5.6 - Modelos usados para previsão quando consideramos que a dependência entre séries é modelada pela LRCM proposta por Kiefer e Vogelsang (2002). 


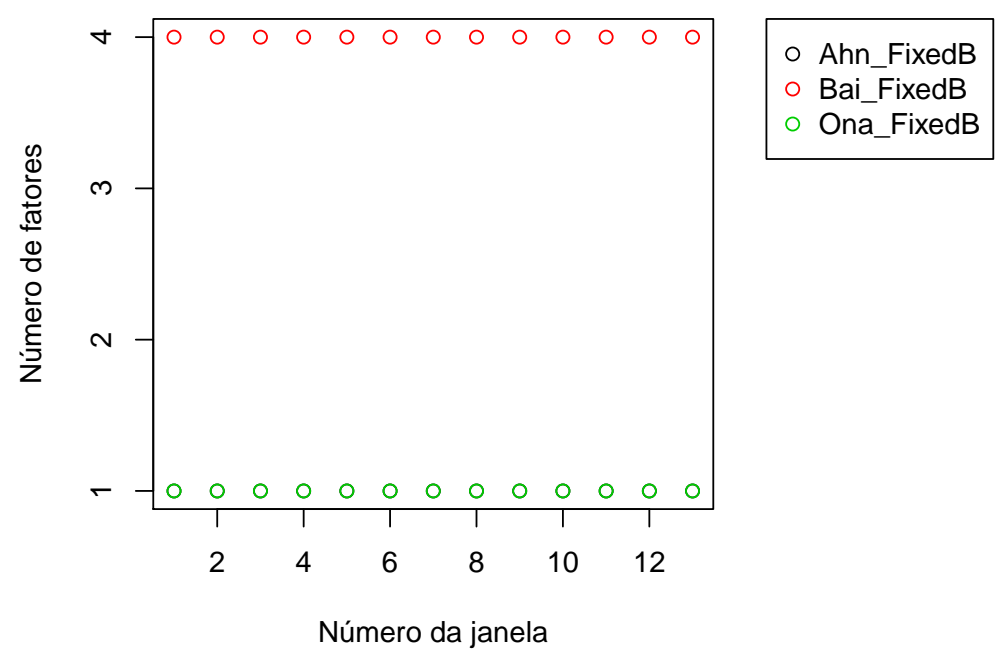

Figura 5.5 - Número de fatores sugeridos pelos critérios apresentado em Ahn e Horenstein (2013),Bai e Ng (2002) e Onatski (2010) quando modelamos a dependência entre a séries por meio da matriz de covariância de longo prazo proposta em Kiefer e Vogelsang (2002)

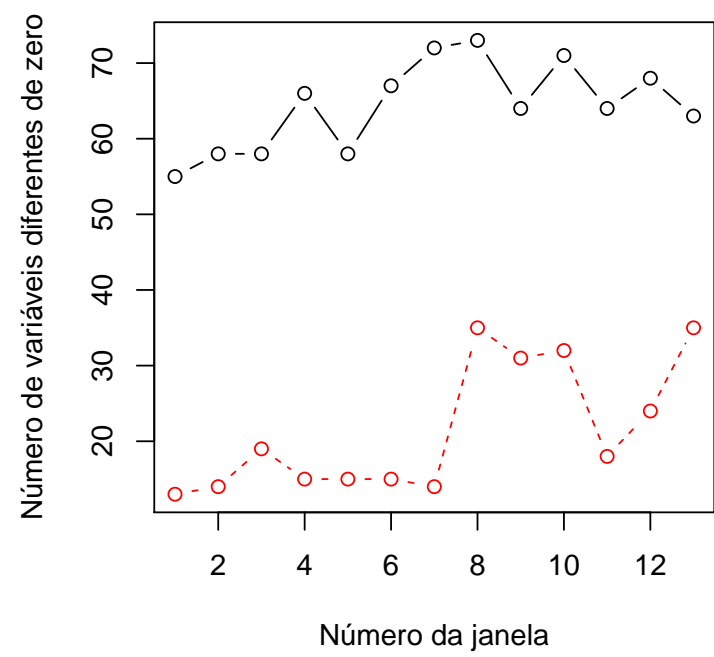

Kristensen

Wang et al

Figura 5.6 - Número de variáveis não nulas sugeridas pelos critérios de Kristensen (2017) e Wang, Li e Leng (2009) quando modelamos a dependência entre a séries por meio da matriz de covariância da classe fixed $b$ proposta por Kiefer e Vogelsang (2002). 


\subsubsection{CMET}

Além dos problemas causados pela possibilidade de dependência temporal, é possível que o acúmulo erros de estimação prejudique o desempenho do modelo. Portanto, consideramos a estimação da matriz de covariância com a aplicação de um hard-threshold. Tanto a matriz como a determinação do limiar podem ser calculados no software R por meio do pacote FinCovRegularization.

Dado que a estrutura de dependência das séries foi alterada, precisamos recalcular o número de fatores latentes e a penalização do SPCA. Os resultados desses cálculos são apresentados nas Figuras 5.7 e 5.8, respectivamente.

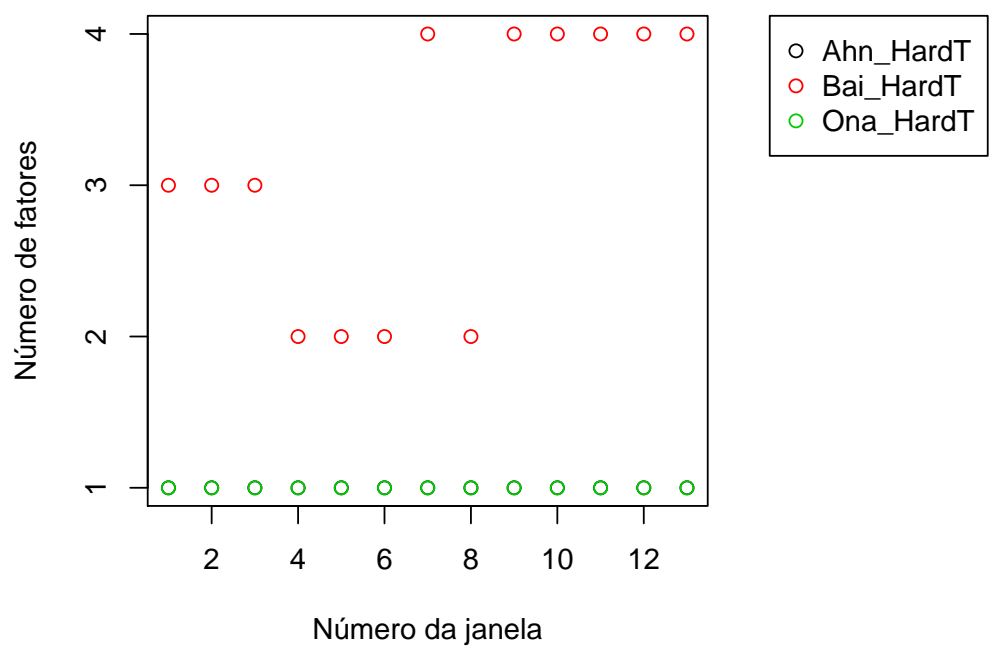

Figura 5.7 - Número de fatores sugeridos pelos critérios apresentados em Ahn e Horenstein (2013), Bai e Ng (2002) e Onatski (2010) quando modelamos a dependência entre a séries por meio da matriz de covariância após a aplicação de um limiar como proposto em Bickel, Levina et al. (2008).

Apesar da alteração para a nova matriz, os critérios de Ahn e Horenstein e Onatski continuam indicando a presença de um único fator latente. Já o critério de Bai e Ng oscila de 2 a 4 fatores. Já a penalização usada no SPCA é próxima a obtida quando modelamos a dependência entre as séries pela matriz de covariância amostral. Esse critérios motivam a estimação dos modelos apresentados na Tabela 5.7. 


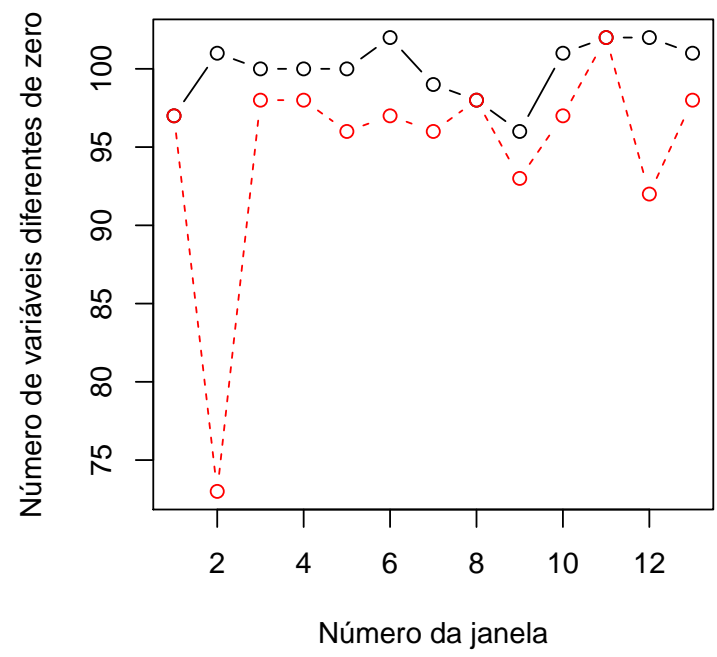

Figura 5.8 - Número de variáveis não nulas sugeridas pelos critérios de Kristensen (2017) e Wang, Li e Leng (2009) quando modelamos a dependência entre a séries por meio da matriz de covariância após a aplicação de um limiar como proposto em Bickel, Levina et al. (2008).

\begin{tabular}{cccc}
\hline ID do Modelo & Método de extração & No de fatores & Penalização \\
\hline M06PC & PCA & $1 \mathrm{~F}$ & - \\
M07PC & PCA & BaiNg & - \\
M10SPC & SPCA & $1 \mathrm{~F}$ & Kristensen \\
M11SPC & SPCA & $1 \mathrm{~F}$ & Wang \\
M12SPC & SPCA & $1 \mathrm{~F}$ & Fixa(98) \\
\hline
\end{tabular}

Tabela 5.7 - Modelos usados para previsão quando consideramos que a dependência entre séries é modelada pela matriz de covariância após a aplicação de um hardthreshold.

\subsubsection{Matriz de Ledoit e Wolf}

Porém, além do acúmulo de erros de estimações, a alta dimensão pode levar a distorções na estrutura de autovalores - autovetores da matriz de covariância amostral. Uma alternativa é impor um regularização aos autovalores como em Ledoit e Wolf (2004).

Note ainda que a matriz de covariância proposta por Ledoit e Wolf (2004) impõe um regularização dos autovalores, mas não altera os autovetores da matriz de covariância amostral. Portanto, é possível que o número de fatores sugeridos pelos critérios de Ahn e Horenstein e Onatski sejam alterado. Logo, recalculamos os critérios após substituir a matriz de covariância amostral. Os resultados desses novos cálculos são apresentados na Figura 5.9.

Nota-se que os critérios sugerem exatamente o mesmo número de fatores do que 


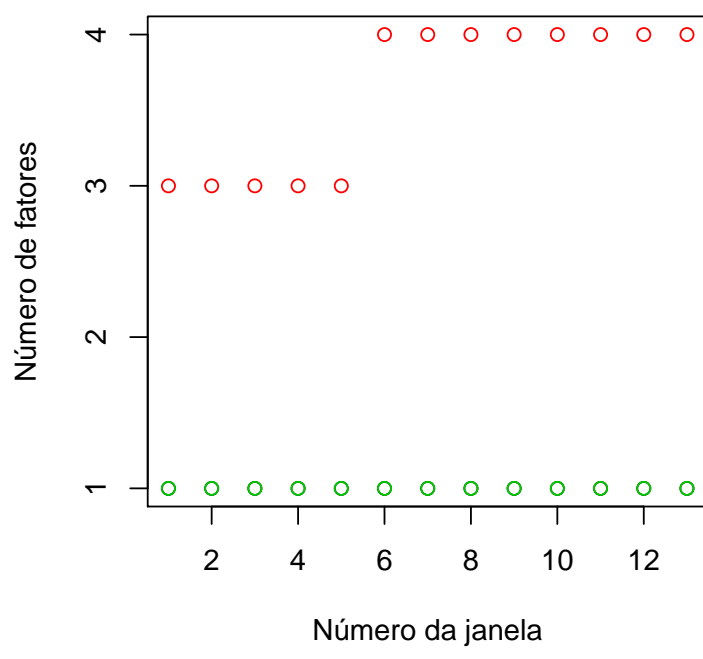

- Ahn_LeW
- Bai_LeW
- Ona_LeW

Figura 5.9 - Número de fatores sugeridos pelos critérios apresentados em Ahn e Horenstein (2013),Bai e Ng (2002) e Onatski (2010) quando modelamos a dependência entre a séries por meio da matriz de covariância proposta em Ledoit e Wolf (2004)

quando consideramos a matriz de covariância amostral. Portanto, não é necessário estimar novos modelos para esse caso.

A partir das matrizes e métodos de extração apresentados anteriormente, estimamos os modelos fatoriais apresentados na Tabela 5.8.

Após a determinação da matriz de covariância, o número de fatores latentes e o número de variáveis diferente de zero, é possível usar PCA e SPCA para extrair os fatores latentes e, em sequência, os demais parâmetros do DFM podem ser estimados via VAR.

Adicionalmente, são estimados 4 modelos adicionais baseados em regressão com penalização e GDFM como mostrado na Tabela 5.9.

Note que o número de choques comuns no GDFM é dada por Hallin, Liska et al. (2007) e o algoritmo usado para o cálculo do número de choques comuns, estimação do GDFM e previsão foi obtido na página pessoal de Matteo Barigozzi. ${ }^{1}$. Já as previsões feitas pelos modelos de regressão com penalização foram obtidas por meio da função ic.glmnet. ${ }^{2}$

\footnotetext{
${ }^{1}$ Veja http://www.barigozzi.eu/Codes.html

${ }^{2}$ Veja https://github.com/gabrielrvsc/HDeconometrics
} 


\begin{tabular}{ccccc}
\hline ID do Modelo & Matriz de covariância & Método de extração & No fatores & Penalização \\
\hline M01PC & Amostral & PCA & $1 \mathrm{~F}$ & - \\
M02PC & Amostral & PCA & BaiNg & - \\
M03PC & Andrews & PCA & $1 \mathrm{~F}$ & - \\
M04PC & Fixed b & PCA & $1 \mathrm{~F}$ & - \\
M05PC & Fixed b & PCA & $4 \mathrm{~F}$ & - \\
M06PC & Hard Threshold & PCA & $1 \mathrm{~F}$ & - \\
M07PC & Hard Threshold & PCA & BaiNg & - \\
M01SPC & Amostral & SPCA & $1 \mathrm{~F}$ & Kristensen \\
M02SPC & Amostral & SPCA & $1 \mathrm{~F}$ & Wang \\
M03SPC & Amostral & SPCA & $1 \mathrm{~F}$ & Fixa(61) \\
M04SPC & Andrews & SPCA & $1 \mathrm{~F}$ & Kristensen \\
M05SPC & Andrews & SPCA & $1 \mathrm{~F}$ & Wang \\
M06SPC & Andrews & SPCA & $1 \mathrm{~F}$ & Fixa(32) \\
M07SPC & Fixed b & SPCA & $1 \mathrm{~F}$ & Kristensen \\
M08SPC & Fixed b & SPCA & $1 \mathrm{~F}$ & Wang \\
M09SPC & Fixed b & SPCA & $1 \mathrm{~F}$ & Fixa(41) \\
M10SPC & Hard Threshold & SPCA & $1 \mathrm{~F}$ & Kristensen \\
M11SPC & Hard Threshold & SPCA & $1 \mathrm{~F}$ & Wang \\
M12SPC & Hard Threshold & SPCA & $1 \mathrm{~F}$ & Fixa(98) \\
\hline
\end{tabular}

Tabela 5.8 - DFM estimados na aplicação empírica.

\begin{tabular}{ccc}
\hline ID do Modelo & Método & Penalização \\
\hline M01CONC & adaLASSO & AIC \\
M02CONC & LASSO & AIC \\
M03CONC & LASSO & BIC \\
M04CONC & GDFM & - \\
\hline
\end{tabular}

Tabela 5.9 - Modelos adaLASSO, LASSO e GDFM estimados na aplicação empírica. 


\subsection{Previsão}

Após a estimação, cada um dos modelos é usado para gerar previsões para os próximos 1, 2, 4, 6 e 8 trimestres para cada uma das variáveis de interesse.

\subsubsection{GDP 251}

Considere, inicialmente, apenas os modelos baseados na matriz de covariância amostral estimados por PCA ou pelo SPCA com número fixo de variáveis na construção dos fatores. Nessa situação as previsões para 1,2,4 e 8 trimestres a frente são apresentadas na Figura 5.10.

Após realizar as previsões, desejamos verificar o desempenho de cada um dos modelos. Para isso usamos o MSFE e o MCS. A Tabela 5.10 indica os modelos que a apresentam o menor MSFE em cada horizonte. No apêndice temos uma tabela indicando o MSFE de cada modelo para cada série analisada em todos os horizontes de tempo.

\begin{tabular}{cccccc}
\hline & FH1 & FH2 & FH4 & FH6 & FH8 \\
\hline GDP251 & M02PC & M01PC & M03SPC & M02PC & M01PC \\
\hline
\end{tabular}

Tabela 5.10 - Modelo com o menor MSFE para cada horizonte de previsão quando consideramos apenas os modelos que usam a matriz de covariância amostral e o método de extração é PCA ou SPCA com número fixo de variáveis não nulas.

Como apresentado na Tabela 5.10, o uso do SPCA leva ao menor valor de MSFE em pelo menos um horizonte de previsão. Esse resultado indica que a introdução de um método que realize alguma forma de seleção, como o SPCA, pode levar a um menor erro de previsão para variáveis macroeconômicas como discutido em Boivin e Ng (2006).

Adicionalmente, assim como discutido em Kristensen (2017), verifica-se que SPCA e PCA levam a valores próximos nas previsões fortalecendo a visão de que o SPCA é uma alternativa razoável ao PCA como método de extração.

Para verificar se os modelos possuem EPA, utilizamos o algoritmo do MCS. Consideramos a matriz de perda quadrática, a estatística TR, um nível de significância de $\alpha=0.2$ e 5000 replicações por bootstrap. Os resultados desse procedimento são apresentados na Tabela 5.11. A cor preta indica que o modelo está presente no conjunto de habilidade preditiva superior. Usaremos essa mesma especificação e forma de apresentação nos demais modelos.

Note que quase todos os modelos analisados nessa primeira situação fazem parte do MCS. Novamente, esse resultado fortalece a visão de Kristensen (2017) de que o SPCA produz previsões competitivas quando comparado ao PCA.

No segundo caso, adicionamos os modelos com matriz de covariância de Andrews e Fixed-b. Tais modelos levam as previsões apresentadas na Figura 5.11. 
GDP251

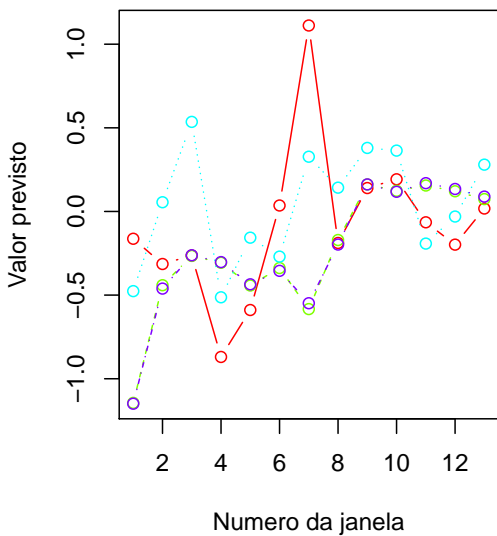

(a) Previsão para 1 trimestre a frente do GDP251

GDP251

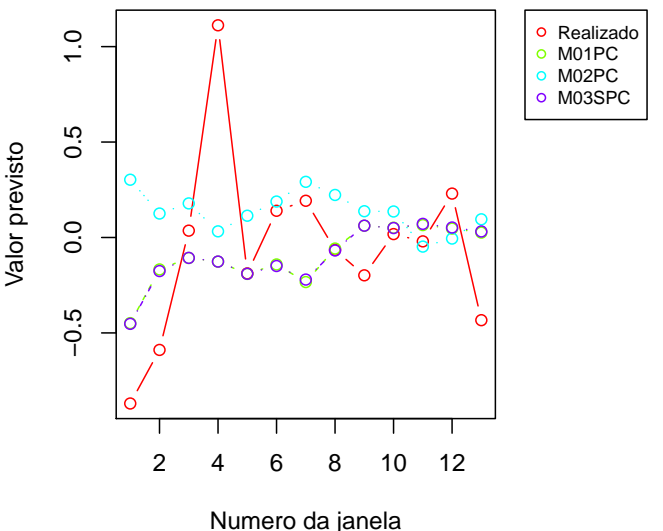

(c) Previsão para 4 trimestres a frente do GDP251
GDP251

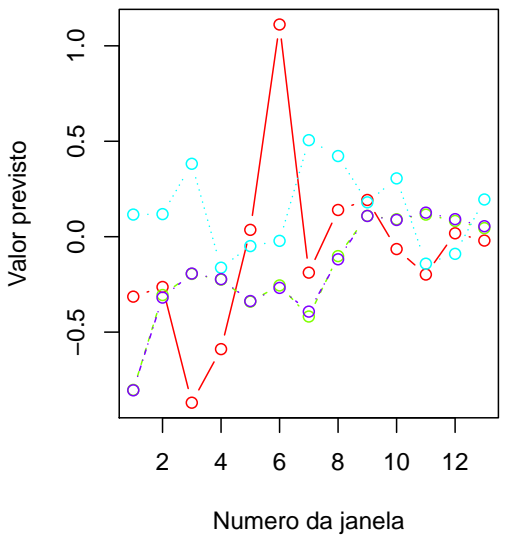

(b) Previsão para 2 trimestres a frente do GDP251

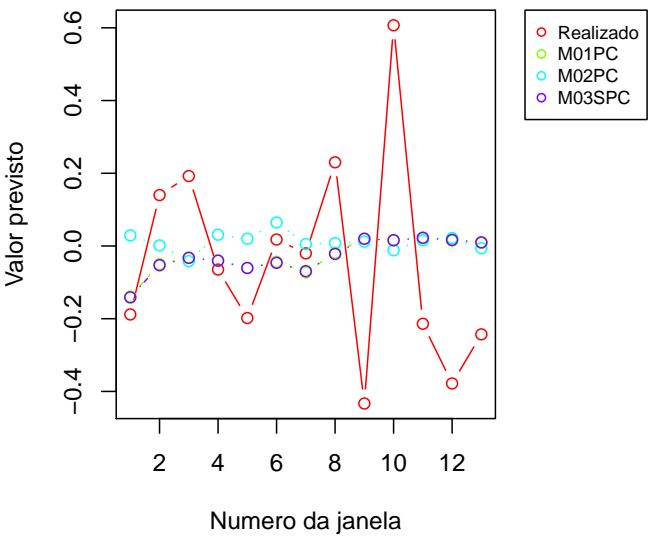

(d) Previsão para 8 trimestres a frente do GDP251

Figura 5.10 - Previsão do GDP251 para os próximos 1,2,4 e 8 trimestres considerando os modelos do caso 1.

\begin{tabular}{llllll}
\hline & FH1 & FH2 & FH4 & FH6 & FH8 \\
\hline M01PC & & & & & \\
M02PC & & & & & \\
M03SPC & & & & & \\
\hline
\end{tabular}

Tabela 5.11 - Resultados do MCS para GDP251 em cada horizonte de previsão quando consideramos apenas os modelos que usam a matriz de covariância amostral e o método de extração é PCA ou SPCA com número fixo de variáveis não nulas. 
GDP251

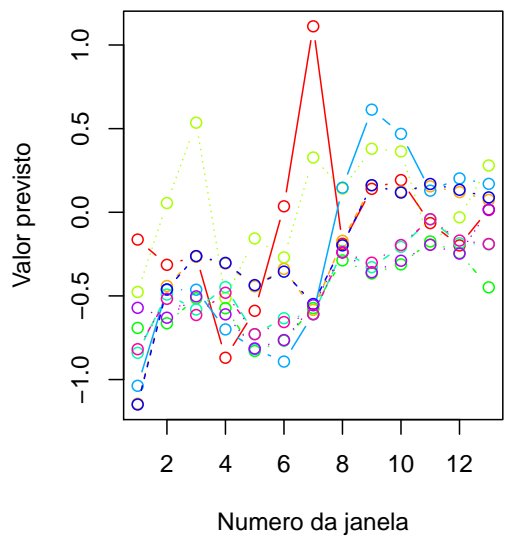

(a) Previsão para 1 trimestre a frente do GDP251

GDP251

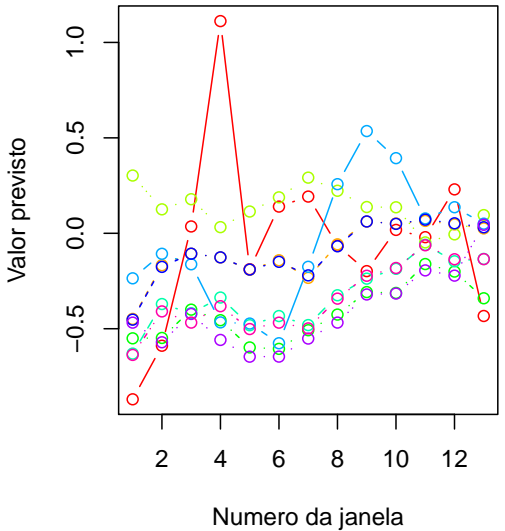

(c) Previsão para 4 trimestres a frente do GDP251
GDP251

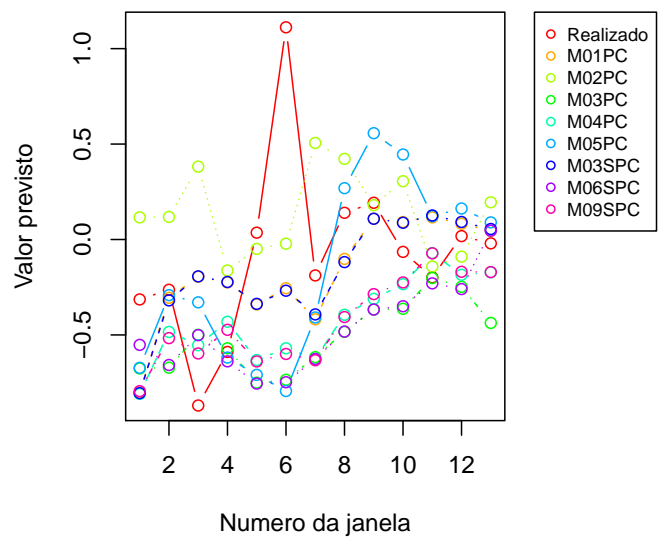

(b) Previsão para 2 trimestres a frente do GDP251

GDP251

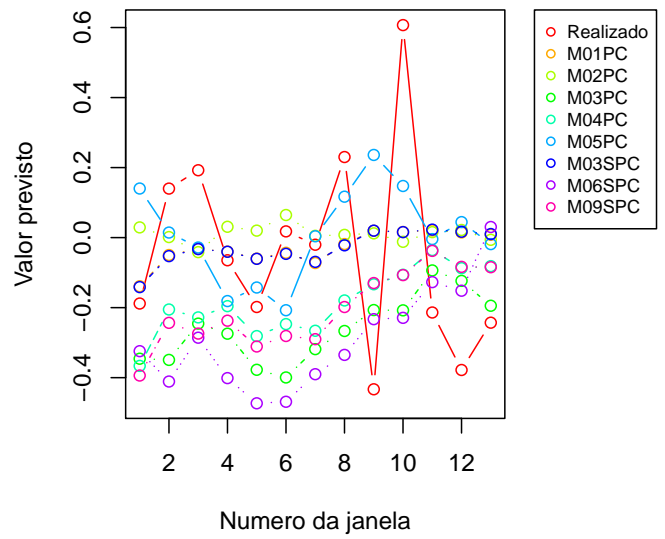

(d) Previsão para 8 trimestres a frente do GDP251

Figura 5.11 - Previsão do GDP251 para os próximos 1,2,4 e 8 trimestres considerando os modelos do caso 2 . 
Para verificar o desempenho preditivo desse conjunto de modelos, apresentamos os modelos com menor MSFE na Tabela 5.12 e os resultados do MCS na Tabela 5.13.

\begin{tabular}{cccccc}
\hline & FH1 & FH2 & FH4 & FH6 & FH8 \\
\hline GDP251 & M02PC & M01PC & M03SPC & M02PC & M01PC
\end{tabular}

Tabela 5.12 - Modelo com o menor MSFE para cada horizonte de previsão quando adicionamos a possibilidade de modelar as séries pelas matrizes de Andrews e Fixed-b e o método de extração é PCA ou SPCA com número fixo de variáveis não nulas.

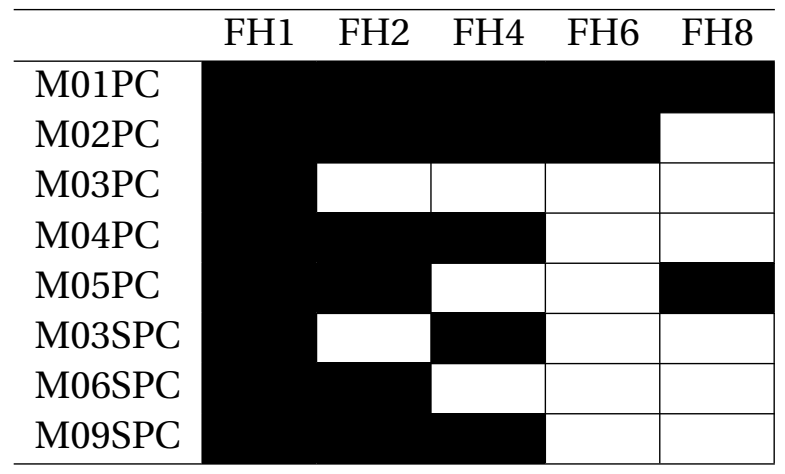

Tabela 5.13 - MCS para cada horizonte de previsão quando adicionamos a possibilidade de modelar as séries pelas matrizes de Andrews e Fixed-b e o método de extração é PCA ou SPCA com número fixo de variáveis não nulas.

Apesar das potenciais vantagens de usar uma LRCM, os modelos com menor MSFE são os mesmos do caso anterior. No entanto, especialmente no curto prazo, o MCS não diferencia entre as habilidade preditivas dos modelos, mas a medida que o horizonte de previsão aumenta, os modelos baseados em componentes principais se destacam em relação a versão com componentes principais esparsas. Por exemplo, para 8 trimestres a frente, $\mathrm{O}$ MCS não rejeita que os modelos M01PC e M05PC possuam a mesma habilidade preditiva.

Mas, além da dependência temporal, a matriz e covariância amostral pode acumular erros de estimações. Portanto, incluímos a possibilidade de usarmos CMET em que o limiar é dado por um hard-threshold. Ao incluir essa possibilidade, temos as previsões apresentadas na Figura 5.12.

Devido a inclusão desses novos modelos, devemos reanalisar o MSFE e MCS. Os novos resultados são apresentados nas Tabelas 5.14 e 5.15.

\begin{tabular}{cccccc}
\hline & FH1 & FH2 & FH4 & FH6 & FH8 \\
\hline GDP251 & M07PC & M01PC & M12SPC & M07PC & M01PC \\
\hline
\end{tabular}

Tabela 5.14 - Modelo com o menor MSFE para cada horizonte de previsão quando adicionamos ao caso anterior a possibilidade de modelar as séries por matrizes de covariância após a aplicação de um limiar 
GDP251

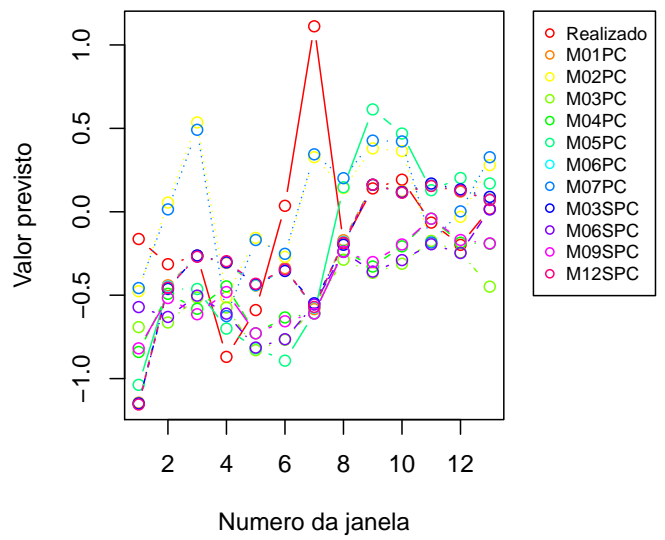

(a) Previsão para 1 trimestre a frente do GDP251

GDP251

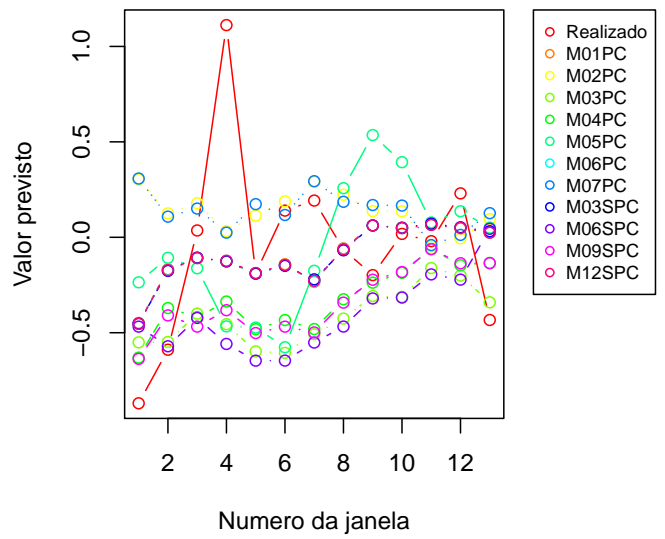

(c) Previsão para 4 trimestres a frente do GDP251

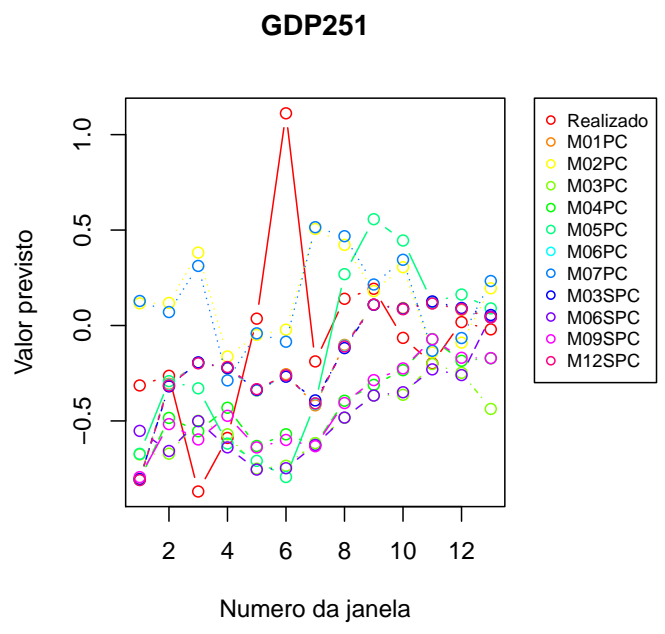

(b) Previsão para 2 trimestres a frente do GDP251

GDP251

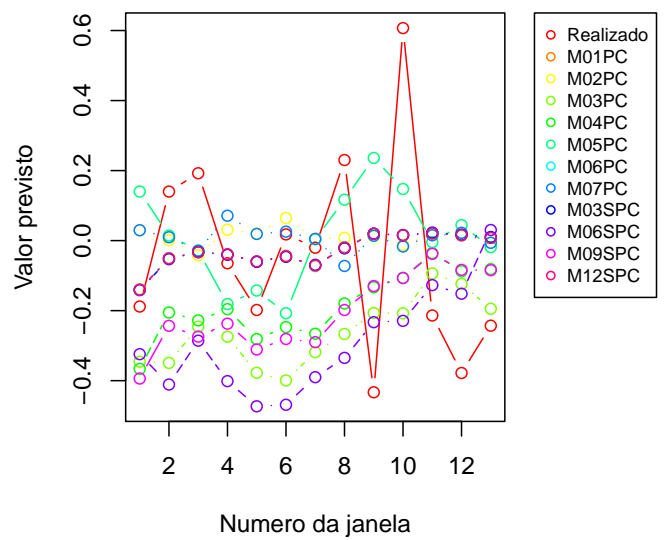

(d) Previsão para 8 trimestres a frente do GDP251

\section{Figura 5.12 - Previsão do GDP251 para os próximos 1,2,4 e 8 trimestres considerando os} modelos do caso 3 . 


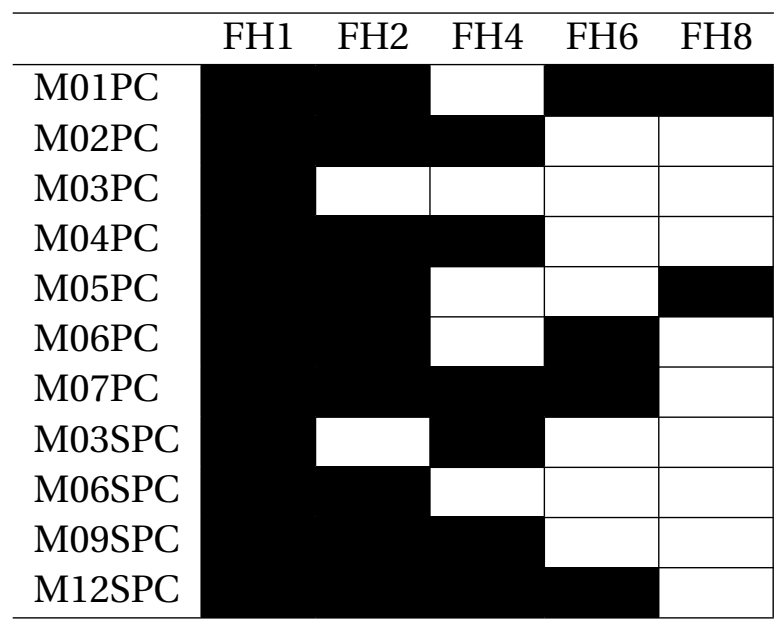

Tabela 5.15 - MCS para cada horizonte de previsão quando adicionamos ao caso anterior a possibilidade de modelar as séries por matrizes de covariância após a aplicação de um limiar

Temos que a classe de CMET leva ao menor MSFE para os casos de previsões 1,4 e 6 trimestres a frente. Tal alteração dos modelos com menor MSFE pode indicar que o acúmulo de erros de estimação na matriz de covariância amostral afeta a sua habilidade de fornecer bons preditores, no entanto o MCS não é capaz de rejeitar a hipótese de EPA entre a maior parte dos modelos. Especialmente no curto prazo, o MCS ainda considera que os modelos possuem a mesma habilidade preditiva, apesar de que para horizontes mais longos, o algoritmo já é capaz de excluir parte dos modelos propostos.

Até o momento, consideramos que os fatores são construídos por um mesmo número de variáveis. No entanto, podemos considerar a possibilidade de que esse número se altere. Nesta situação, temos a inclusão de novos modelos levando as previsões apresentadas na Figura 5.13.

Com essa nova possibilidade reavaliamos o MSFE e o MCS. Os novos resultados são apresentados nas Tabelas 5.18 e 5.19 .

\begin{tabular}{cccccc}
\hline & FH1 & FH2 & FH4 & FH6 & FH8 \\
\hline GDP251 & M07PC & M01PC & M11SPC & M07PC & M01PC \\
\hline
\end{tabular}

Tabela 5.16 - Modelo para GDP251 com o menor MSFE para cada horizonte de previsão quando consideramos as matrizes de covariância amostral, Andrews, fixed-b, matriz após a aplicação de um limiar e o método de extração pode ser PCA ou SPCA.

Essa alteração leva alteração do modelo com menor MSFE para 4 trimestres a frente, porém , os modelos com menor MSFE para os demais horizontes são mantidos.

Com essa nova versão, agora temos modelos baseados em SPCA presentes no conjunto de habilidade preditiva superior para horizontes maiores. Por exemplo, M01SPC e M02SPC 
GDP251

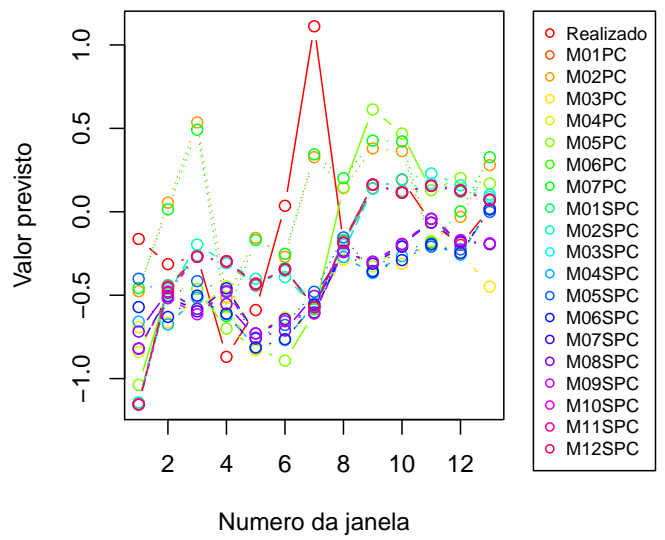

(a) Previsão para 1 trimestre a frente do GDP251

GDP251

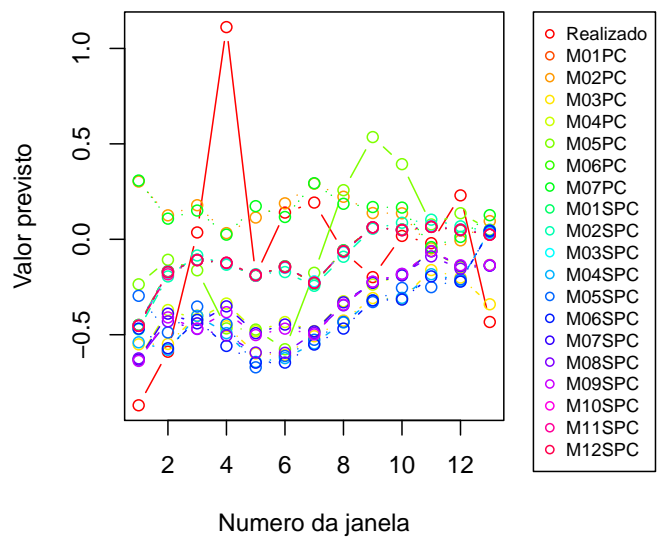

(c) Previsão para 4 trimestres a frente do GDP251
GDP251

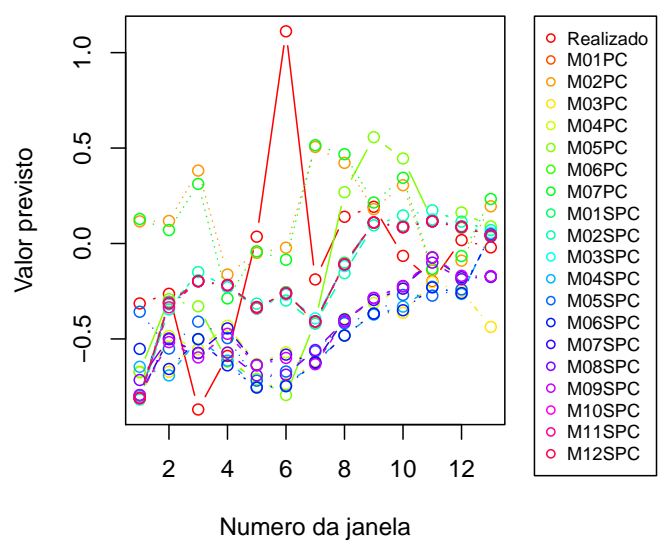

(b) Previsão para 2 trimestres a frente do GDP251

GDP251

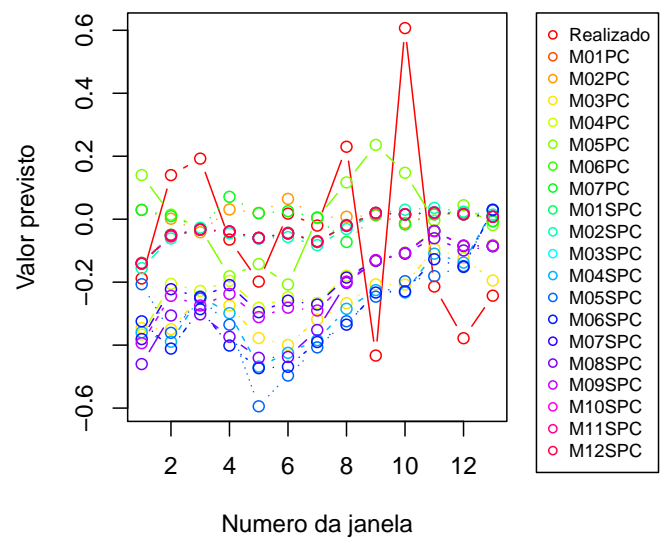

(d) Previsão para 8 trimestres a frente do GDP251

\section{Figura 5.13 - Previsão do GDP251 para os próximos 1,2,4 e 8 trimestres considerando os} modelos do caso 4 . 


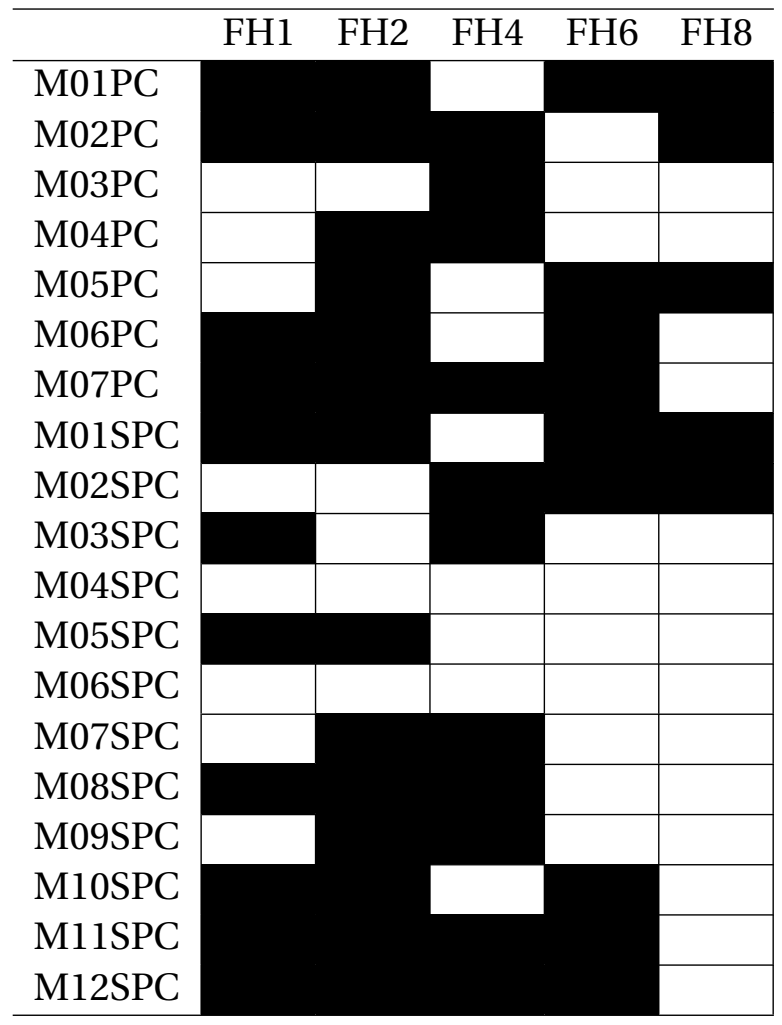

Tabela 5.17 - MCS para cada horizonte de previsão quando consideramos as matrizes de covariância amostral, Andrews, fixed-b, matriz após a aplicação de um limiar e o método de extração pode ser PCA ou SPCA.

fazem parte do conjunto de habilidade preditiva superior para previsões feitas 8 trimestres a frente para GDP251.

Por fim, consideramos os modelos concorrentes baseados em regressões com penalização e GDFM. Nesse caso, as previsões são apresentadas na Figura 5.14.

Similarmente aos casos anteriores, os modelos com menor MSFE e os resultados do MCS são apresentados nas Tabelas 5.18 e 5.19.

\begin{tabular}{cccccc}
\hline & FH1 & FH2 & FH4 & FH6 & FH8 \\
\hline GDP251 & M07PC & M04CONC & M11SPC & M04CONC & M03CONC \\
\hline
\end{tabular}

Tabela 5.18 - Modelo com o menor MSFE para cada horizonte de previsão quando consideramos todos os modelos apresentados na seção anterior.

Os resultados indicam o bom desempenho do GDFM. Esse modelo apresenta o menor MSFE para 2 e 6 trimestres a frente. Apesar do melhor desempenho em termos de MSFE, o algoritmo do MCS não é capaz de rejeitar a hipótese de EPA para grande parte dos modelos, apenas considerando que alguns dos modelos baseados em regressões penalizadas e o GDFM fazem parte do conjunto de habilidade preditiva superior. 
GDP251

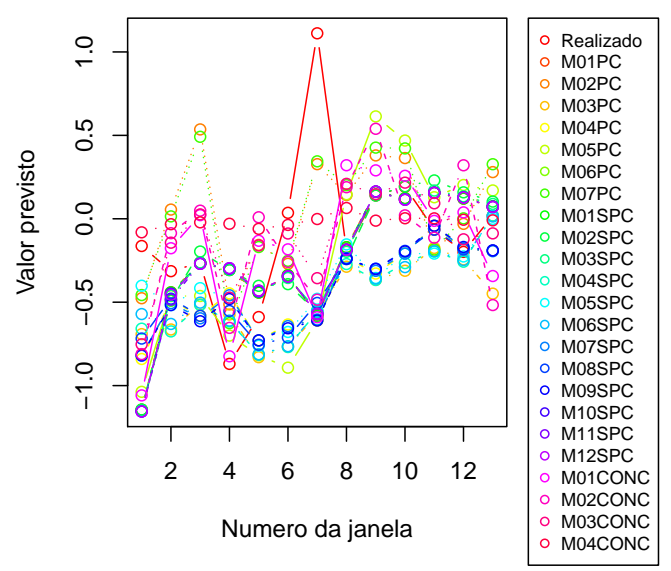

(a) Previsão para 1 trimestre a frente do GDP251

GDP251

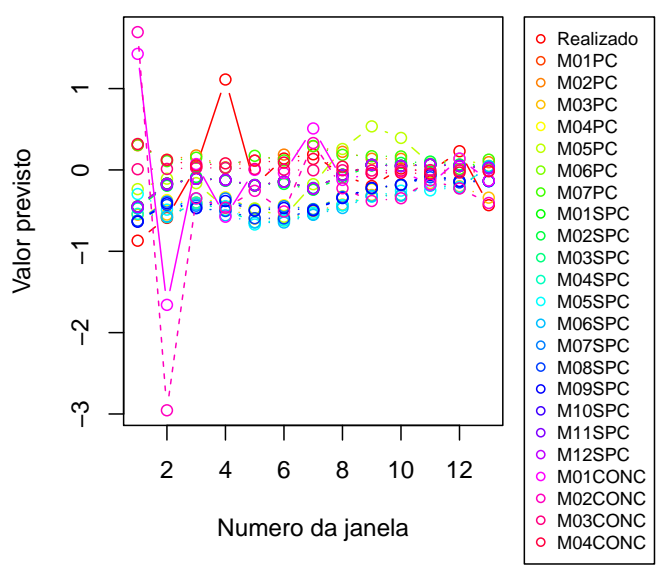

(c) Previsão para 4 trimestres a frente do GDP251
GDP251

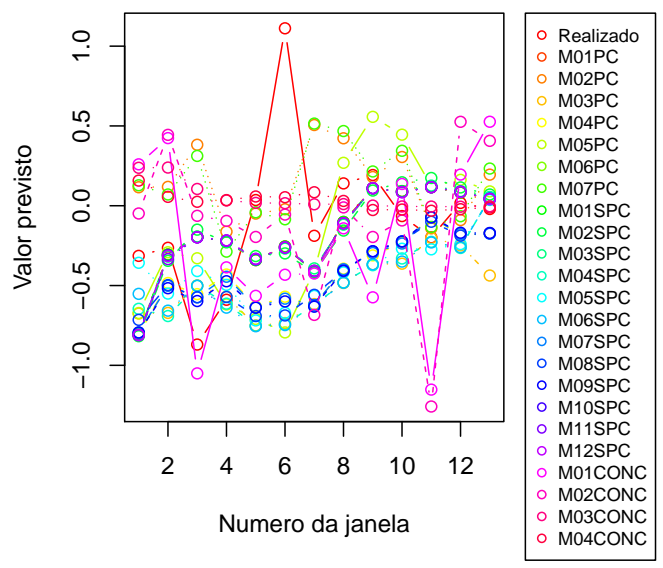

(b) Previsão para 2 trimestres a frente do GDP251

GDP251

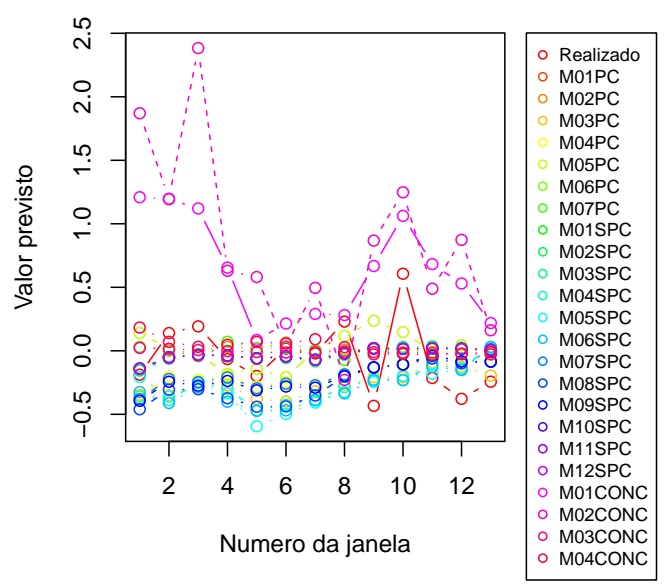

(d) Previsão para 8 trimestres a frente do GDP251

\section{Figura 5.14 - Previsão do GDP251 para os próximos 1,2,4 e 8 trimestres considerando os} modelos do caso 5 . 


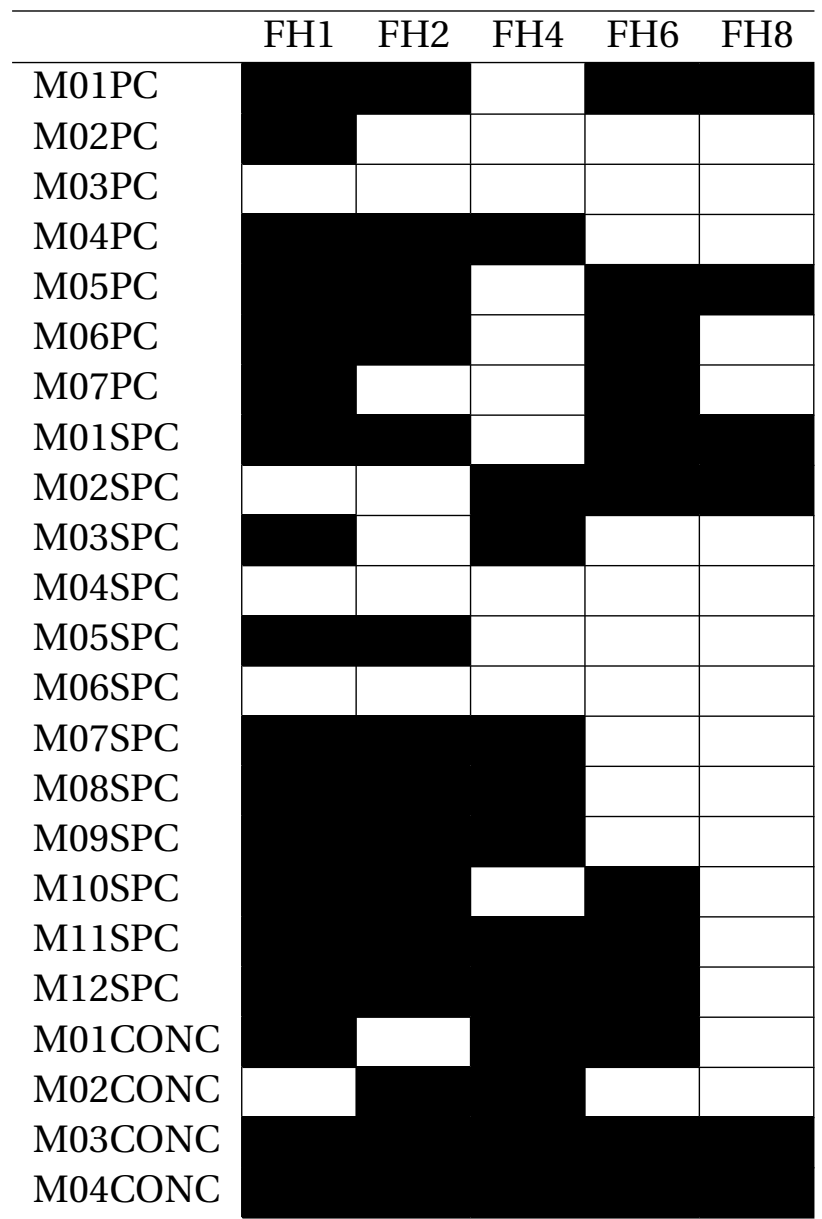

Tabela 5.19 - MCS para cada horizonte de previsão quando consideramos todos os modelos apresentados na seção anterior. 
IPS10

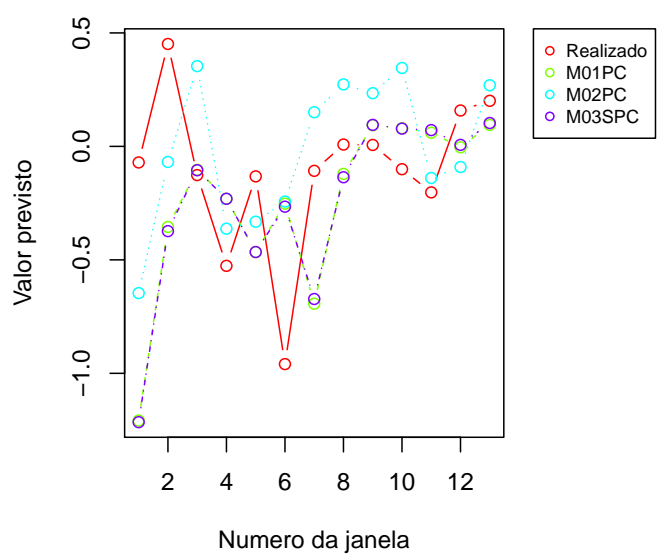

(a) Previsão para 1 trimestre a frente do IPS10

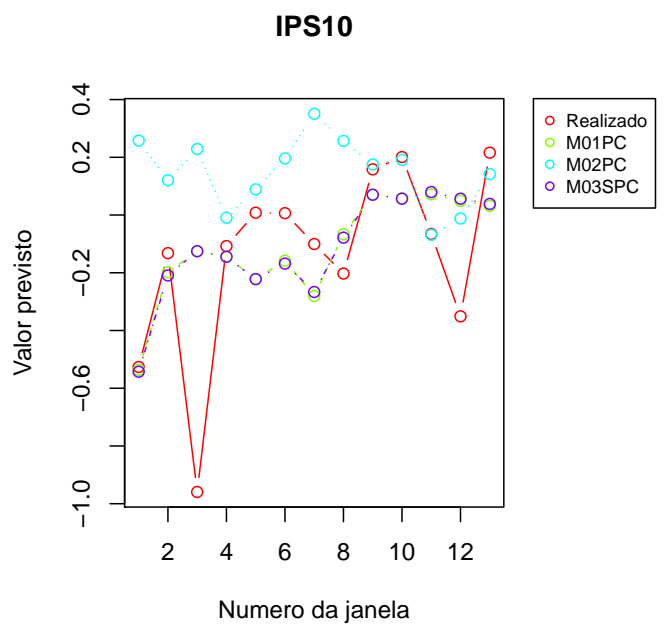

(c) Previsão para 4 trimestres a frente do IPS10

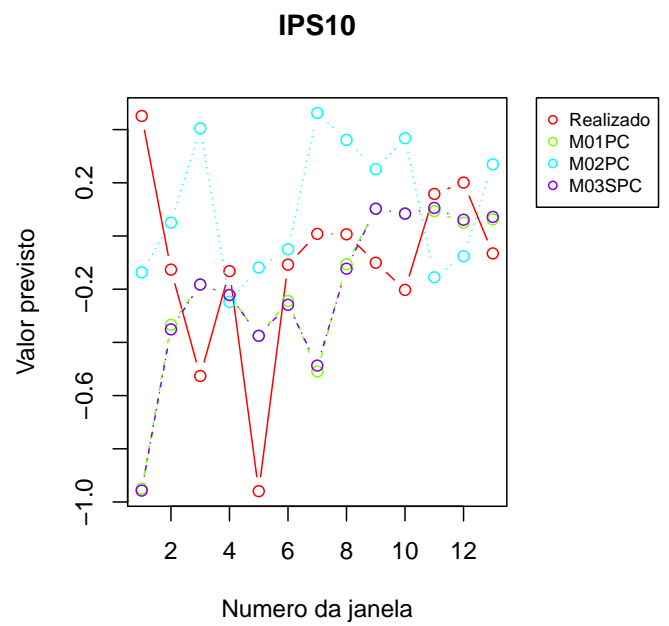

(b) Previsão para 2 trimestres a frente do IPS10

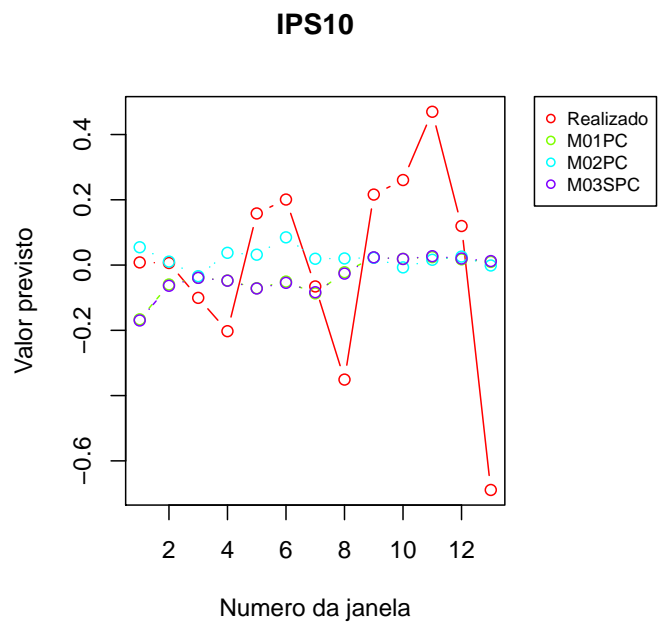

(d) Previsão para 8 trimestres a frente do IPS10

Figura 5.15 - Previsão do IPS10 para os próximos 1,2,4 e 8 trimestres considerando os modelos do caso 1.

\subsubsection{IPS 10}

Similarmente a análise feita para o GDP251, considere, inicialmente, apenas os modelos baseados na matriz de covariância amostral estimados por PCA ou pelo SPCA com número fixo de variáveis na construção dos fatores. As previsões obtidas para o IPS10 nessa situação são apresentadas na Figura 5.15.

Os modelos com menor MSFE para cada horizonte de previsão são apresentados na Tabela 5.20 enquanto os resultados do MCS são apresentados na Tabela 5.21.

Apesar de valores distintos para MSFE, o MCS não rejeita a hipótese nula de EPA 


\begin{tabular}{cccccc}
\hline & FH1 & FH2 & FH4 & FH6 & FH8 \\
\hline IPS10 & M02PC & M01PC & M01PC & M03SPC & M02PC \\
\hline
\end{tabular}

Tabela 5.20 - Modelos para o IPS10 com o menor MSFE para cada horizonte de previsão quando consideramos apenas os modelos que usam a matriz de covariância amostral e o método de extração é PCA ou SPCA com número fixo de variáveis não nulas.

\begin{tabular}{llllll}
\hline & FH1 & FH2 & FH4 & FH6 & FH8 \\
\hline M01PC & & & & & \\
M02PC & & & & & \\
M03SPC & & & & & \\
\hline
\end{tabular}

Tabela 5.21 - MCS para o IPS10 para cada horizonte de previsão quando consideramos apenas os modelos que usam a matriz de covariância amostral e o método de extração é PCA ou SPCA com número fixo de variáveis não nulas.

para a maior parte dos modelos. Novamente, consideramos a possibilidade de alterar a matriz de covariância para versões de LRCM com o intuito de mitigar os efeitos de possíveis dependências temporais. Os resultados das previsões nesse novo contexto são dadas pela Figura 5.16.

Ao avaliarmos os efeitos da inclusão desses modelos, constatamos que os modelos com os menores MSFE são os mesmo do caso anterior, porém os métodos que usam LRCM também constituem parte do conjunto de habilidade preditiva superior construído pelo algoritmo do MCS como indicado nas Tabelas 5.22 e 5.23.

\begin{tabular}{cccccc}
\hline & FH1 & FH2 & FH4 & FH6 & FH8 \\
\hline IPS10 & M02PC & M01PC & M01PC & M03SPC & M02PC \\
\hline
\end{tabular}

Tabela 5.22 - Modelos para IPS10 com o menor MSFE para cada horizonte de previsão quando adicionamos a possibilidade de modelar as séries pelas matrizes de Andrews e Fixed-b e o método de extração é PCA ou SPCA com número fixo de variáveis não nulas.

Assim como no caso do GDP251, consideramos também a possibilidade de usarmos matrizes de covariância após a utilização de um limiar. As previsões desse caso são apresentadas na Figura 5.17.

Para avaliarmos o impacto dessa possibilidades, construímos as Tabelas 5.24 e 5.25.

Nesse contexto, verificamos que a utilização de CMET leva aos modelos com menor MSFE para 3 horizontes de previsão sendo que em dois desses casos a CMET é combinada com a extração por SPCA.

Tal resultado, indica que a CMET, assim como no caso do GDP251 é uma alternativa razoável para modelar a dependência entre séries temporais macroeconômicas uma vez que é capaz de mitigar erros de estimação na matriz de covariância amostral. Ao combina-la com 
IPS10

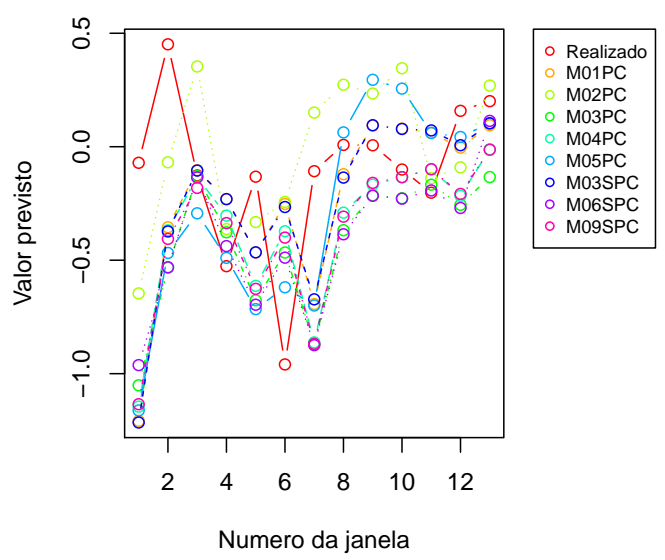

(a) Previsão para 1 trimestre a frente do IPS10

IPS10

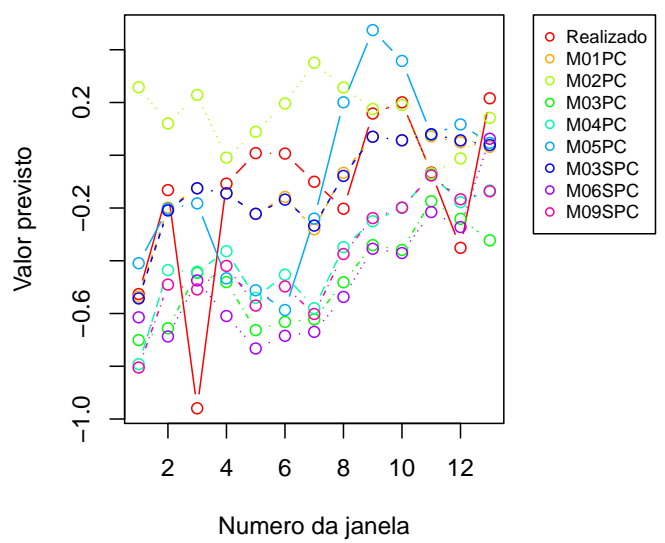

(c) Previsão para 4 trimestres a frente do IPS10
IPS10

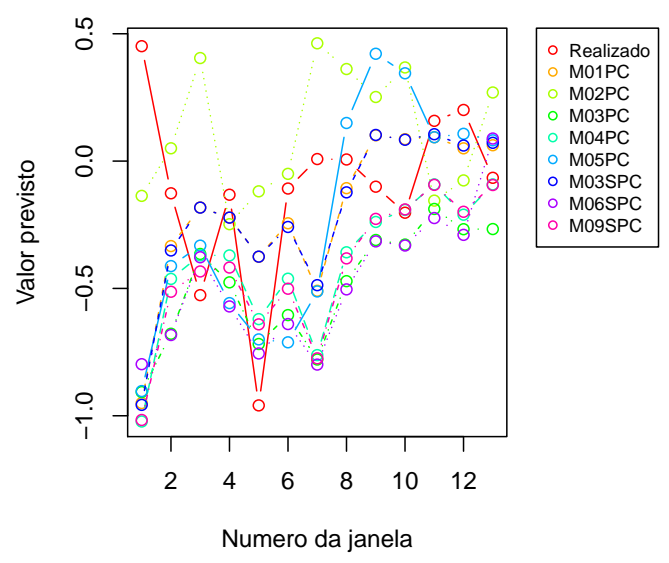

(b) Previsão para 2 trimestres a frente do IPS10

IPS10

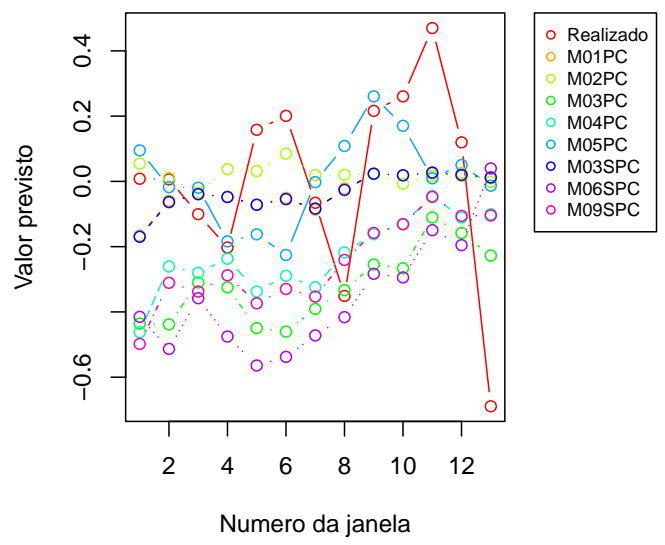

(d) Previsão para 8 trimestres a frente do IPS10

Figura 5.16 - Previsão do IPS10 para os próximos 1,2,4 e 8 trimestres considerando os modelos do caso 2 . 


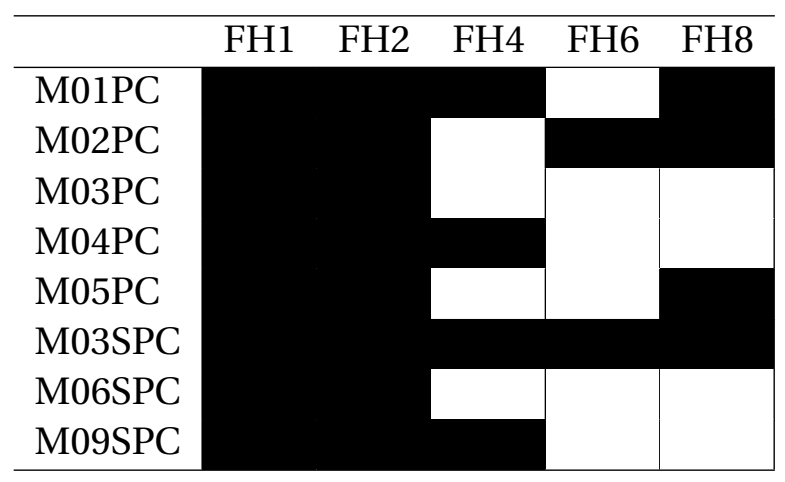

Tabela 5.23 - MCS para IPS10 para cada horizonte de previsão quando adicionamos a possibilidade de modelar as séries pelas matrizes de Andrews e Fixed-b e o método de extração é PCA ou SPCA com número fixo de variáveis não nulas.

\begin{tabular}{cccccc}
\hline & FH1 & FH2 & FH4 & FH6 & FH8 \\
\hline IPS10 & M07PC & M01PC & M12SPC & M03SPC & M07SPC \\
\hline
\end{tabular}

Tabela 5.24 - Modelo para IPS10 com o menor MSFE para cada horizonte de previsão quando adicionamos ao caso anterior a possibilidade de modelar as séries por matrizes de covariância após a aplicação de um limiar

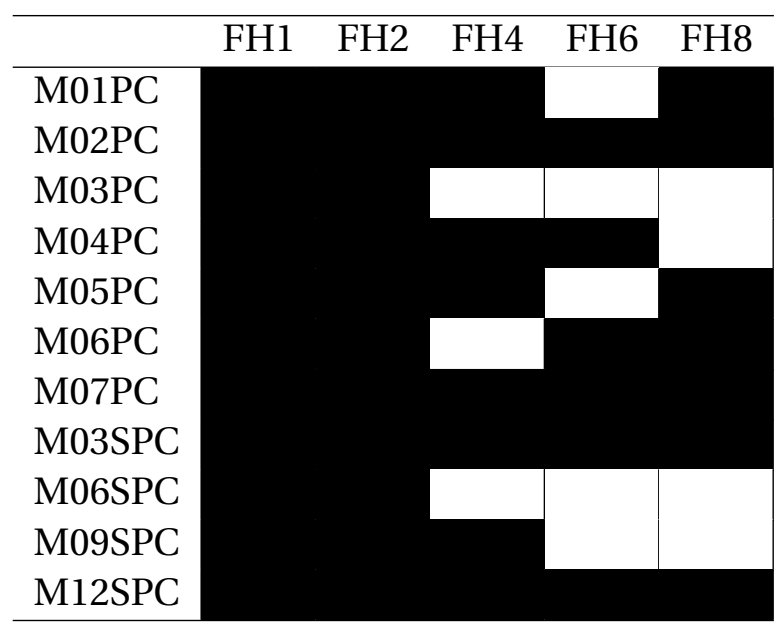

Tabela 5.25 - MCS para IPS10 para cada horizonte de previsão quando adicionamos ao caso anterior a possibilidade de modelar as séries por matrizes de covariância após a aplicação de um limiar 
IPS10

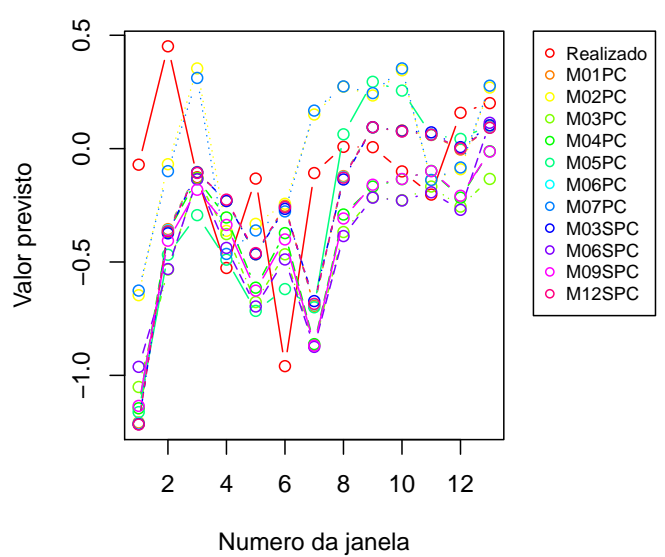

(a) Previsão para 1 trimestre a frente do IPS10

IPS10

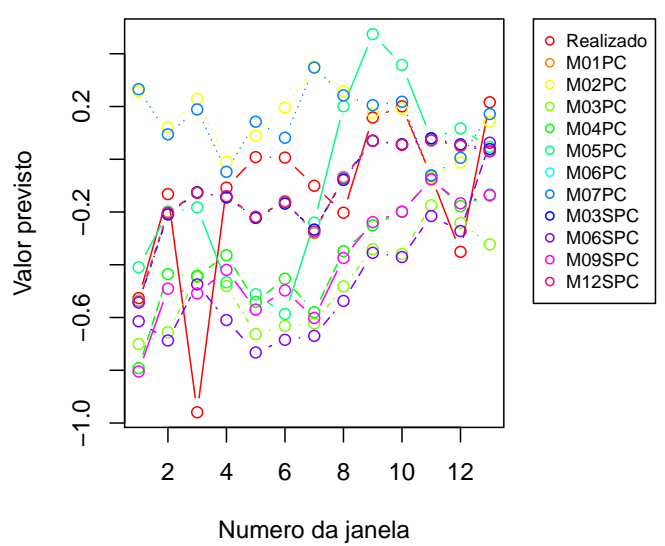

(c) Previsão para 4 trimestres a frente do IPS10
IPS10

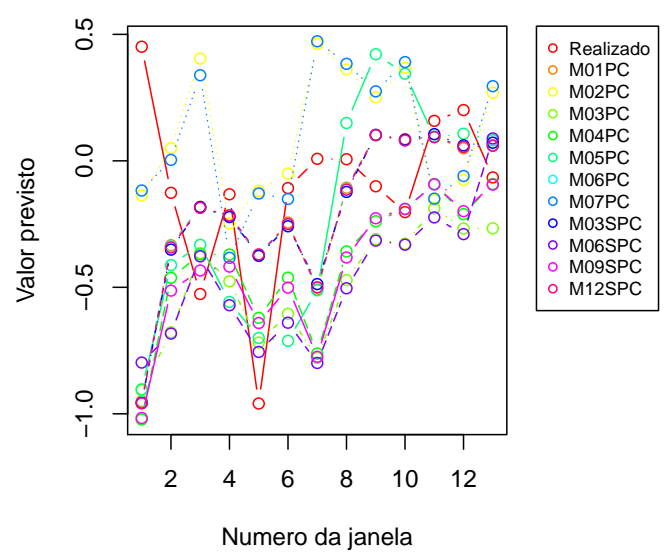

(b) Previsão para 2 trimestres a frente do IPS10

IPS10

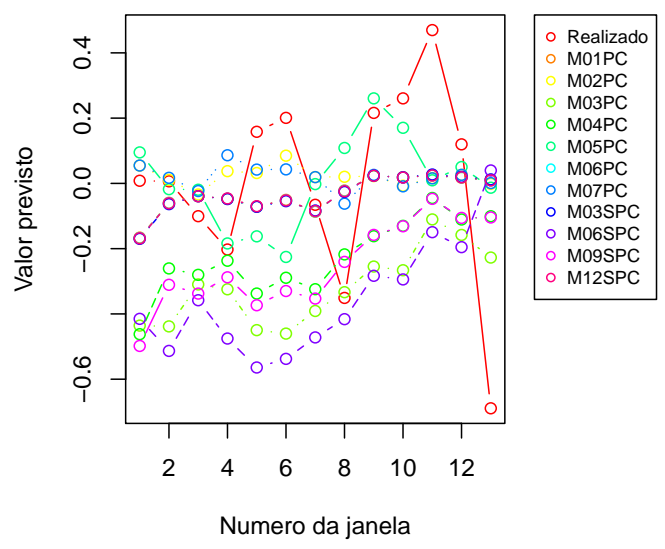

(d) Previsão para 8 trimestres a frente do IPS10

Figura 5.17 - Previsão do IPS10 para os próximos 1,2,4 e 8 trimestres considerando os modelos do caso 3 . 


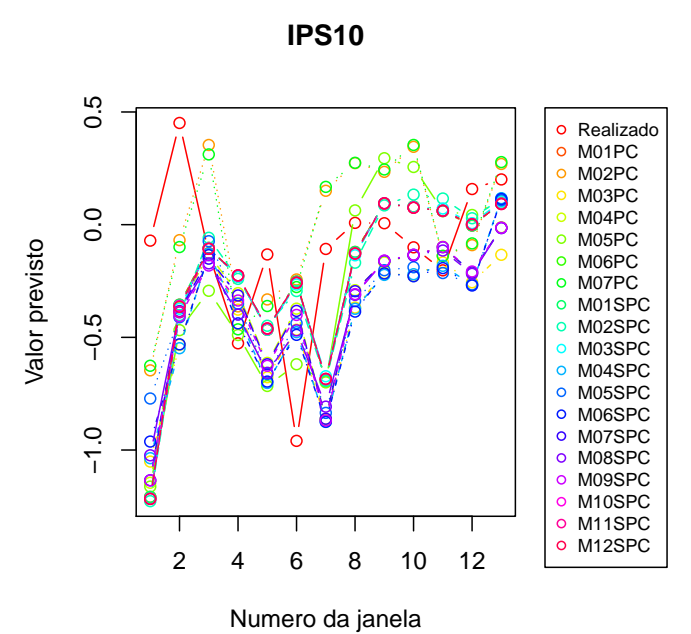

(a) Previsão para 1 trimestre a frente do IPS10

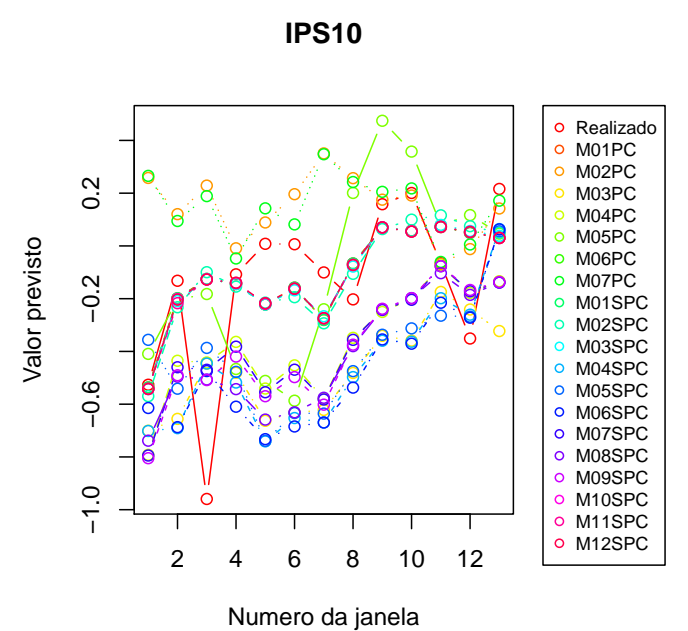

(c) Previsão para 4 trimestres a frente do IPS10

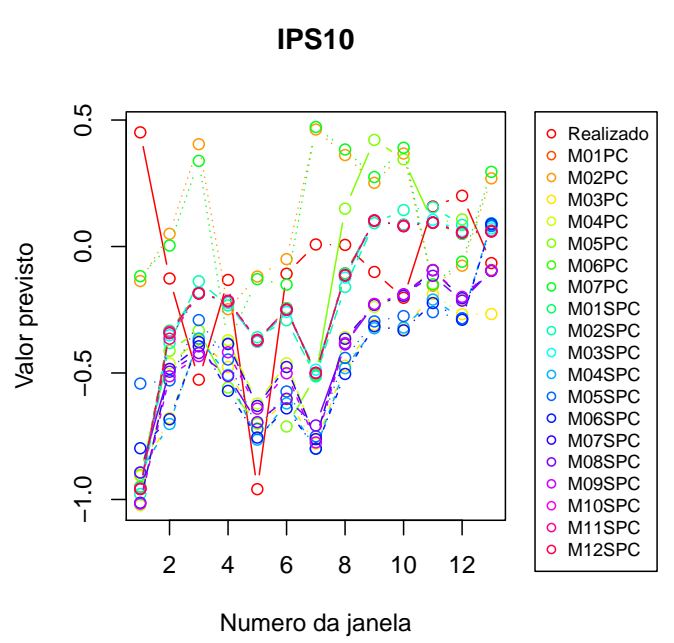

(b) Previsão para 2 trimestres a frente do IPS10

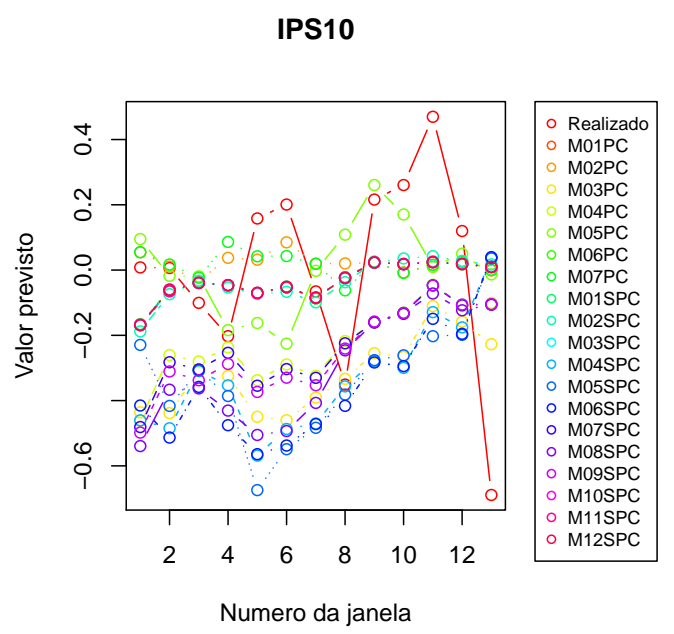

(d) Previsão para 8 trimestres a frente do IPS10

Figura 5.18 - Previsão do IPS10 para os próximos 1,2,4 e 8 trimestres considerando os modelos do caso 4.

o SPCA, é possível removermos variáveis ruidosas que também poderiam influenciar nas previsões. Note também que a combinação de CMET e SPC, por meio do modelo M12SPC, aparece no conjunto dos modelos com capacidade preditiva superior para previsões em todos os horizontes de tempos.

Consideramos a possibilidade de que o número de variáveis usadas na construção do fator se altere. Essa possibilidade leva as previsões apresentadas na Figura 5.18.

Essa mudança, como indicado pela Tabela 5.26, leva a alteração dos modelos com menor MSFE para FH1 e FH6. Mas, assim como anteriormente, o MCS tem dificuldade em rejeitar H0 de EPA para grande parte dos modelos como indicado na Tabela 5.27. 


\begin{tabular}{cccccc}
\hline & FH1 & FH2 & FH4 & FH6 & FH8 \\
\hline IPS10 & M07PC & M05SPC & M12SPC & M02SPC & M07PC \\
\hline
\end{tabular}

Tabela 5.26 - Modelo para IPS10 com o menor MSFE para cada horizonte de previsão quando consideramos as matrizes de covariância amostral, Andrews, Fixed-B, matriz após a aplicação de um limiar e o método de extração pode ser PCA ou SPCA.

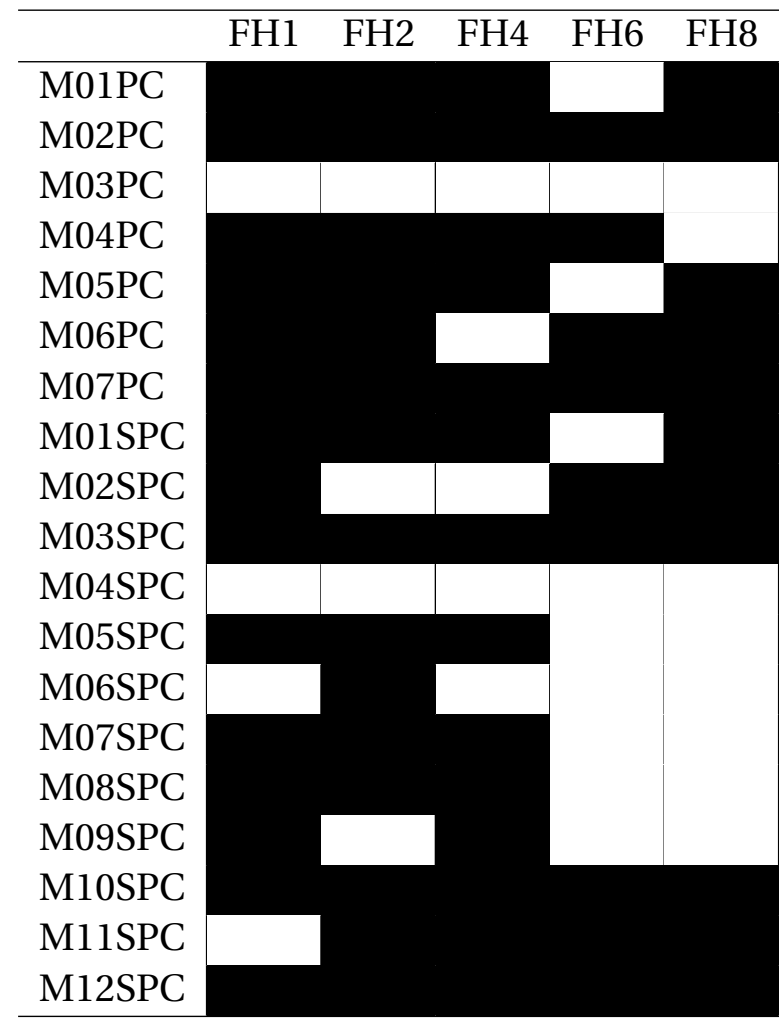

Tabela 5.27 - MCS para IPS10 para cada horizonte de previsão quando consideramos as matrizes de covariância amostral, Andrews, Fixed-B, matriz após a aplicação de um limiar e o método de extração pode ser PCA ou SPCA.

Por fim, consideramos as previsões dos modelos baseados em regressões penalizadas e GDFM conforme apresentado na Figura 5.19.

Assim como no caso do GDP251, podemos observar o bom desempenho do GDFM. Tal modelo apresenta o menor MSFE para previsões 1 e 2 trimestres a frente como indicado na Tabela 5.28. Adicionalmente, podemos verificar que modelos baseado em regressões com penalidade, como o M03CONC, estão presente no MCS como representado na Tabela 5.29.

\begin{tabular}{cccccc}
\hline & FH1 & FH2 & FH4 & FH6 & FH8 \\
\hline IPS10 & M04CONC & M04CONC & M12SPC & M02SPC & M07PC \\
\hline
\end{tabular}

Tabela 5.28 - Modelo para IPS10 com o menor MSFE para cada horizonte de previsão quando consideramos todos os modelos apresentados na seção anterior. 
IPS10

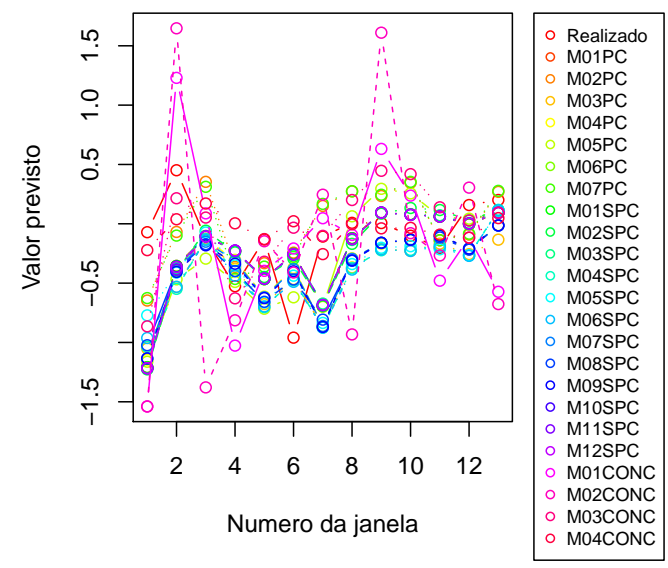

(a) Previsão para 1 trimestre a frente do IPS10

IPS10

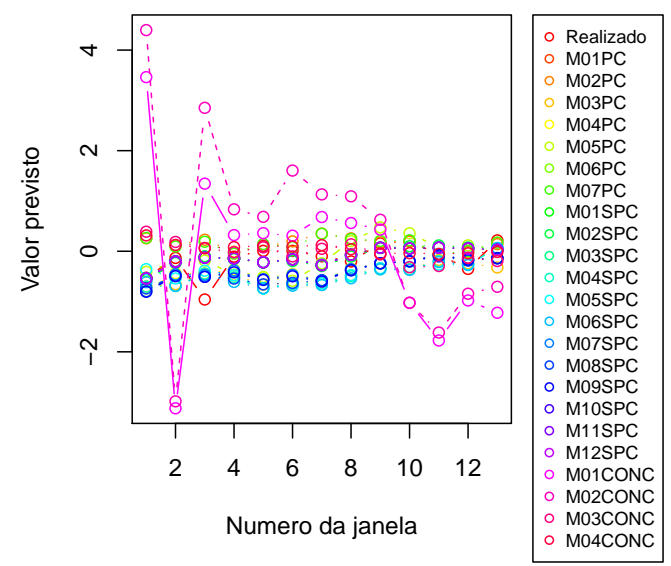

(c) Previsão para 4 trimestres a frente do IPS10
IPS10

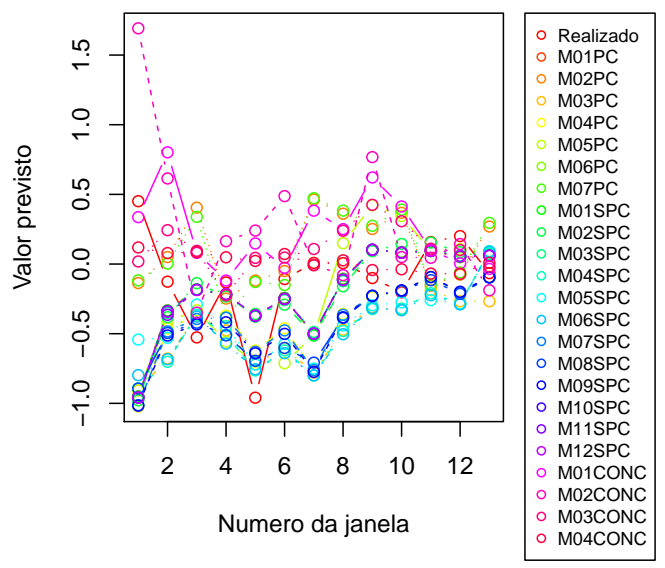

(b) Previsão para 2 trimestres a frente do IPS10

IPS10

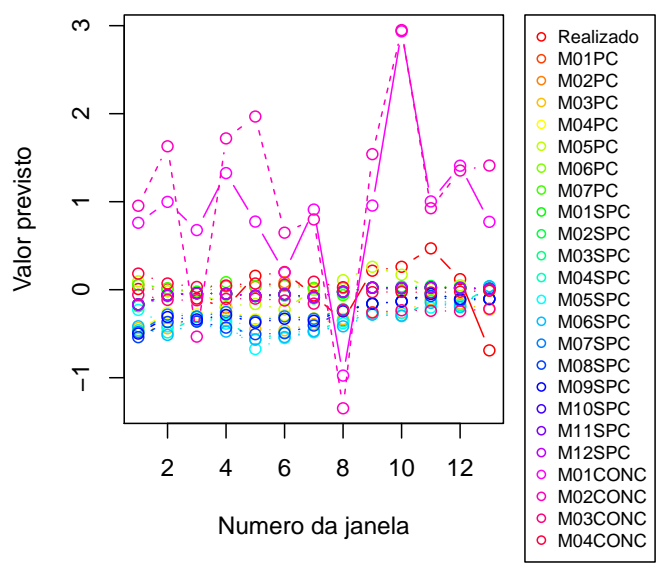

(d) Previsão para 8 trimestres a frente do IPS10

Figura 5.19 - Previsão do IPS10 para os próximos 1,2,4 e 8 trimestres considerando os modelos do caso 5 . 


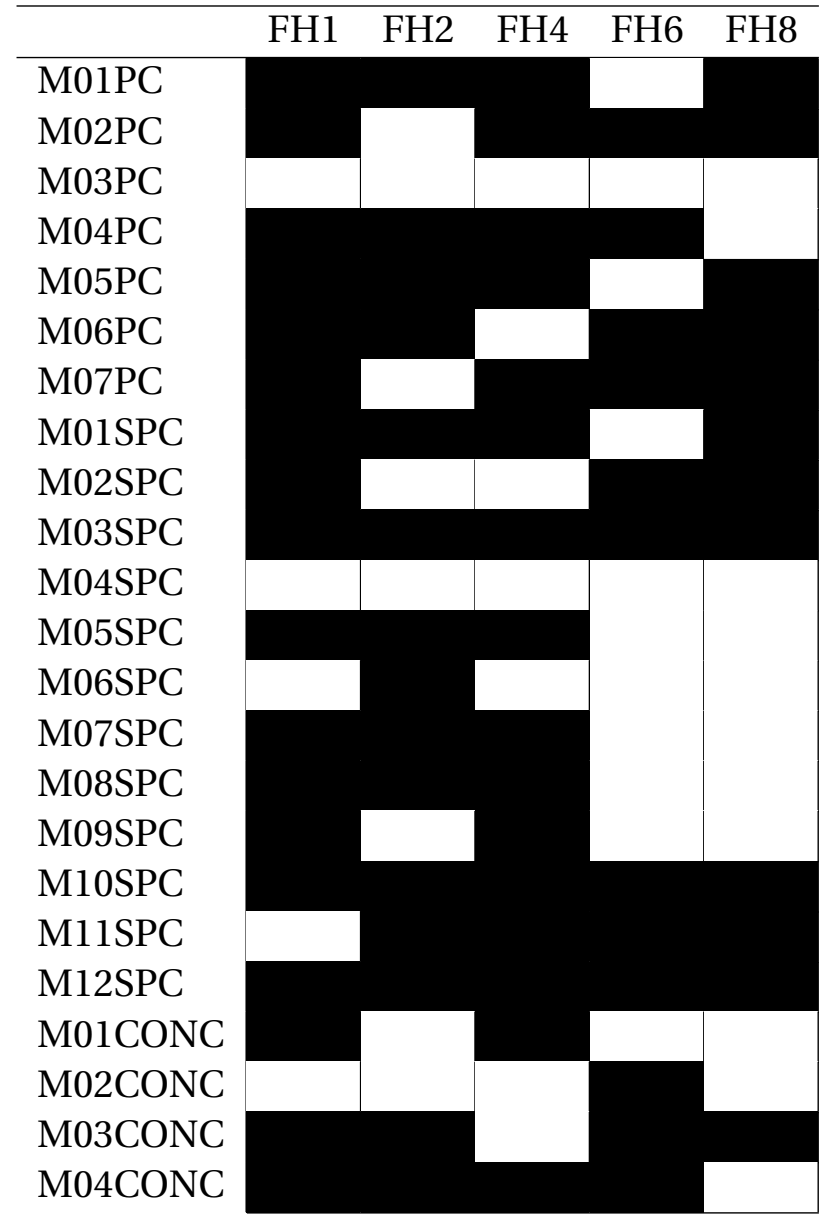

Tabela 5.29 - MCS para IPS10 para cada horizonte de previsão quando consideramos todos os modelos apresentados na seção anterior. 


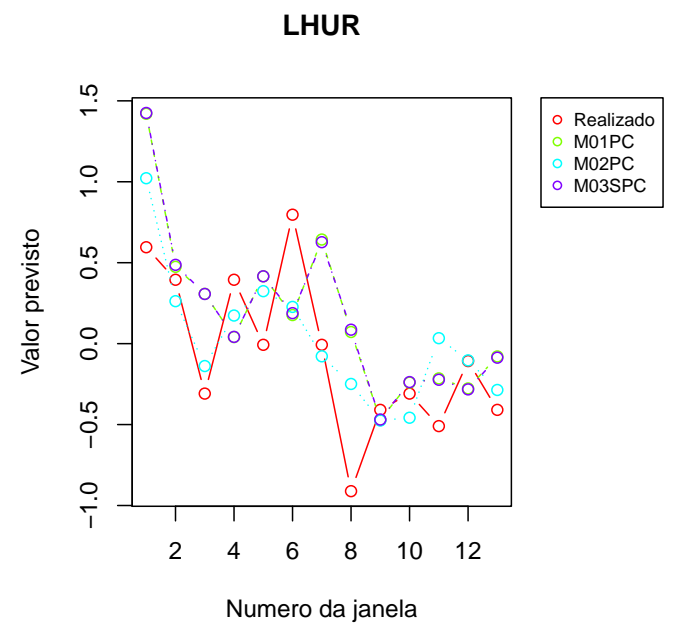

(a) Previsão para 1 trimestre a frente do LHUR

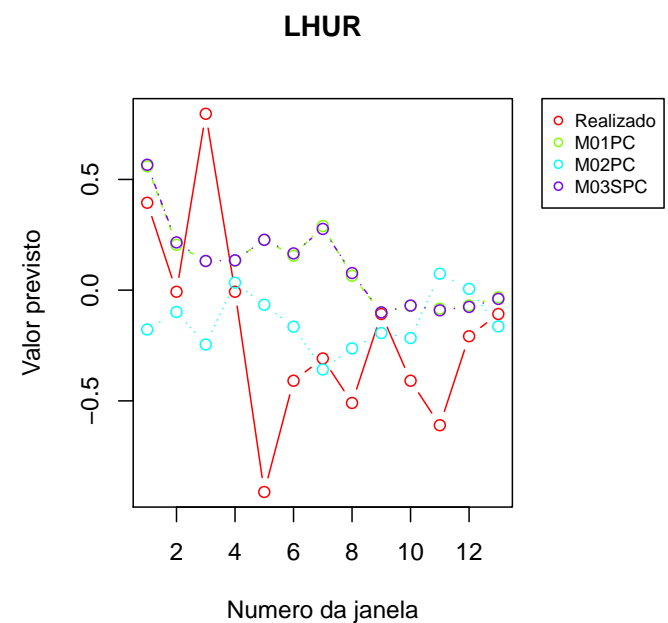

(c) Previsão para 4 trimestres a frente do LHUR

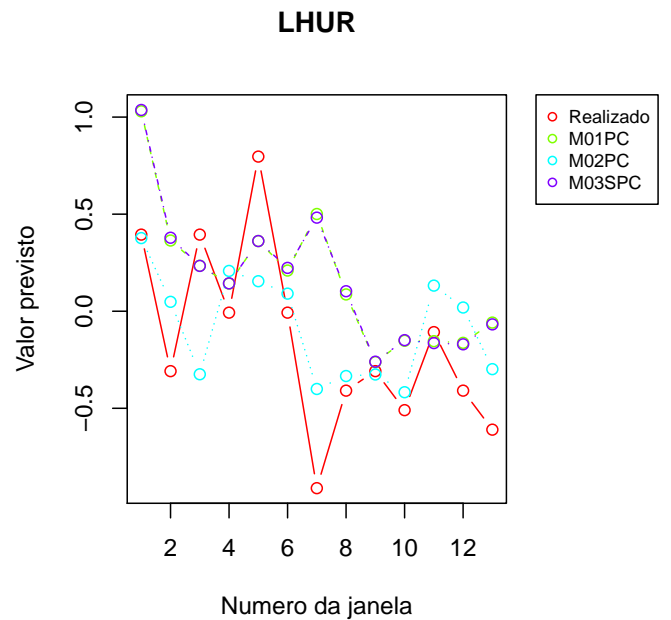

(b) Previsão para 2 trimestres a frente do LHUR

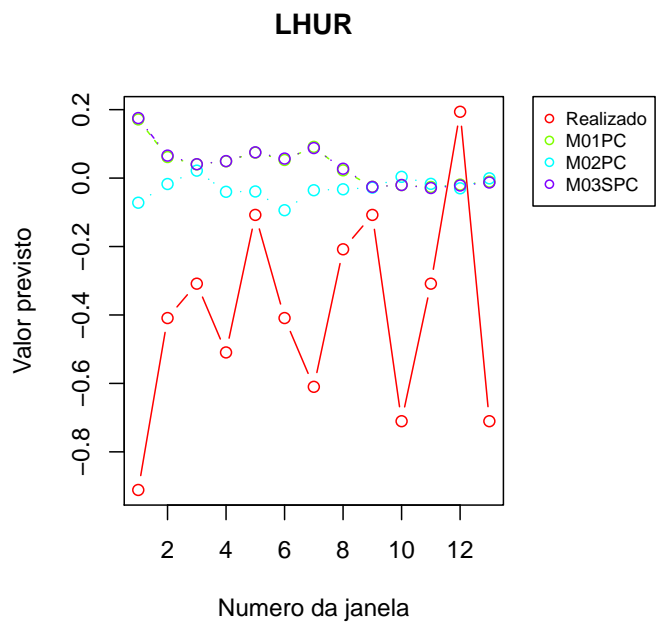

(d) Previsão para 8 trimestres a frente do LHUR

Figura 5.20 - Previsão do LHUR para os próximos 1,2,4 e 8 trimestres considerando os modelos do caso 1.

\subsubsection{LHUR}

Assim como para GDP251 e IPS10, considere os modelos baseados na matriz de covariância amostral estimados por PCA ou pelo SPCA com número fixo de variáveis na construção dos fatores. As previsões obtidas para o IPS10 nessa situação são apresentadas na Figura 5.20. Os demais modelos serão tratados no decorrer dessa sub-sessão.

Ao avaliarmos o MSFE e os resultados do MCS, por meio das Tabelas 5.30 e 5.31, temos que o modelo M02PC é o único pertencente ao conjunto de modelos com habilidade preditiva superior para FH1 e FH8. Tal situação é diferente do que ocorria com GDP251 e IPS10, em 
que em todos os horizontes de previsão para esse caso contemplavam múltiplos modelos no MCS.

\begin{tabular}{cccccc}
\hline & FH1 & FH2 & FH4 & FH6 & FH8 \\
\hline LHUR & M02PC & M02PC & M02PC & M02PC & M02PC \\
\hline
\end{tabular}

Tabela 5.30 - Modelos para LHUR com o menor MSFE para cada horizonte de previsão quando consideramos apenas os modelos que usam a matriz de covariância amostral e o método de extração é PCA ou SPCA com número fixo de variáveis não nulas.

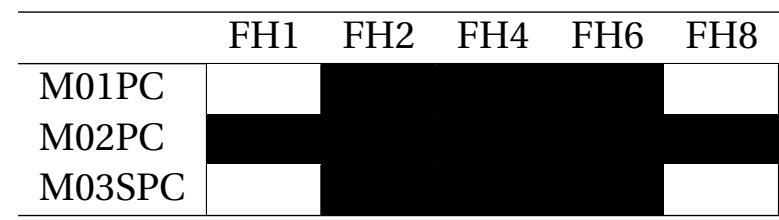

Tabela 5.31 - MCS para LHUR para cada horizonte de previsão quando consideramos apenas os modelos que usam a matriz de covariância amostral e o método de extração é PCA ou SPCA com número fixo de variáveis não nulas.

Assim como para as variáveis macroeconômicas analisadas anteriormente, consideramos a substituição da matriz de covariância amostral pelas matrizes de longo prazo propostas por Andrews (1991) e Kiefer e Vogelsang (2002).

Apesar de M02PC ainda ser o modelo com menor MSFE para a maior parte dos horizontes de previsão, de acordo com a Tabela 5.32, o modelo M05PC baseado na LRCM do tipo fixed-b apresenta o menor MSFE para previsões 8 trimestre a frente e, como indicado na Tabela 5.33, está presente no MCS em 4 dos 5 horizontes de previsão.

\begin{tabular}{cccccc}
\hline & FH1 & FH2 & FH4 & FH6 & FH8 \\
\hline LHUR & M02PC & M02PC & M02PC & M02PC & M05PC \\
\hline
\end{tabular}

Tabela 5.32 - Modelos para LHUR com o menor MSFE para cada horizonte de previsão quando adicionamos a possibilidade de modelar as séries pelas matrizes de Andrews e Fixed-b e o método de extração é PCA ou SPCA com número fixo de variáveis não nulas.

Adicionalmente, consideramos a possibilidade de CMET. As previsões decorrentes dessa inclusão são apresentadas na Figura 5.22 .

Como apresentado na Tabela 5.34, matrizes estimadas após a aplicação de um hardthreshold apresentam um bom desempenho em termos de MSFE. Conforme representado na Tabela 5.35, o modelo M07PC, que usa uma CMET, está no MCS para todos os horizontes de previsão. Nota-se também a presença do modelo M12SPC no MCS. Tal modelo combina CMET com SPC e reforça o bom desempenho dessa combinação assim como foi observado para IPS10. 
LHUR

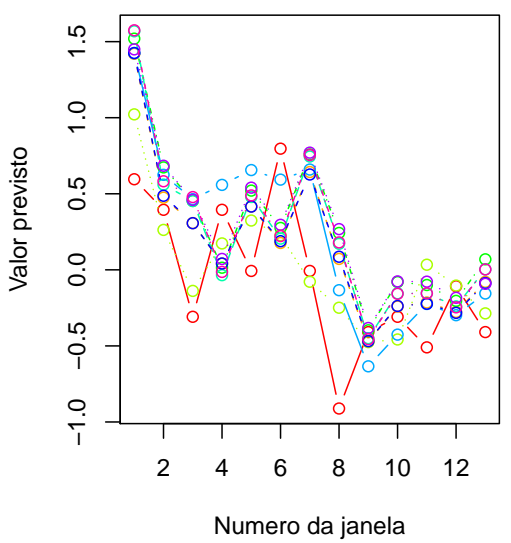

(a) Previsão para 1 trimestre a frente do LHUR

LHUR

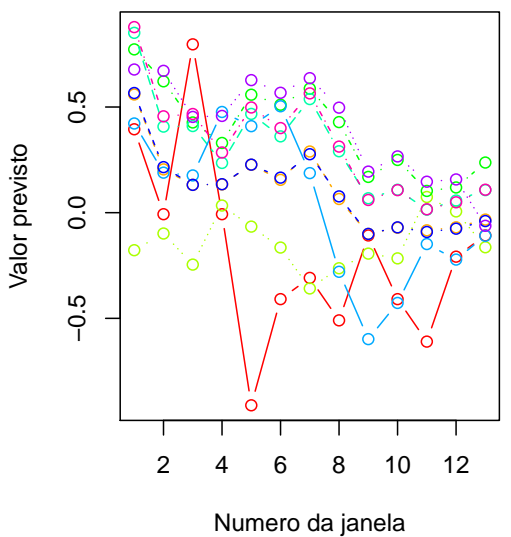

(c) Previsão para 4 trimestres a frente do LHUR
LHUR

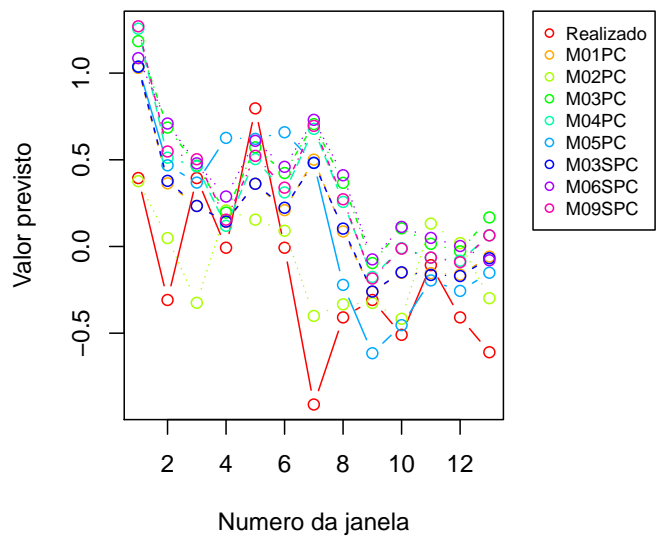

(b) Previsão para 2 trimestres a frente do LHUR

LHUR

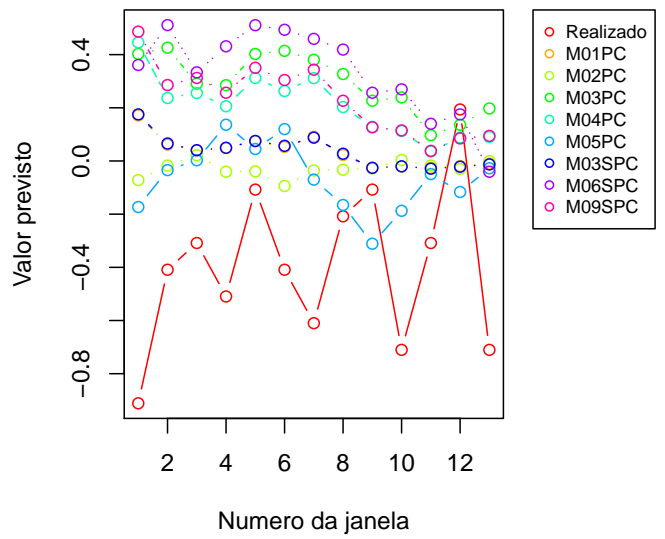

(d) Previsão para 8 trimestres a frente do LHUR

Figura 5.21 - Previsão do LHUR para os próximos 1,2,4 e 8 trimestres considerando os modelos do caso 2. 


\begin{tabular}{|c|c|c|c|c|}
\hline \multirow{2}{*}{ M01PC } & FH1 & FH2 & FH4 & \multirow[t]{2}{*}{ H6 } \\
\hline & & & & \\
\hline M02PC & & & & \\
\hline М03РC & & & & \\
\hline M04PC & & & & \\
\hline M05PC & & & & \\
\hline M03SPC & & & & \\
\hline M06SPC & & & & \\
\hline M09SPC & & & & \\
\hline
\end{tabular}

Tabela 5.33 - MCS para LHUR para cada horizonte de previsão quando adicionamos a possibilidade de modelar as séries pelas matrizes de Andrews e Fixed-b e o método de extração é PCA ou SPCA com número fixo de variáveis não nulas.

\begin{tabular}{cccccc}
\hline & FH1 & FH2 & FH4 & FH6 & FH8 \\
\hline LHUR & M07PC & M07PC & M07PC & M02PC & M05PC \\
\hline
\end{tabular}

Tabela 5.34 - Modelo para LHUR com o menor MSFE para cada horizonte de previsão quando adicionamos ao caso anterior a possibilidade de modelar as séries por matrizes de covariância após a aplicação de um limiar

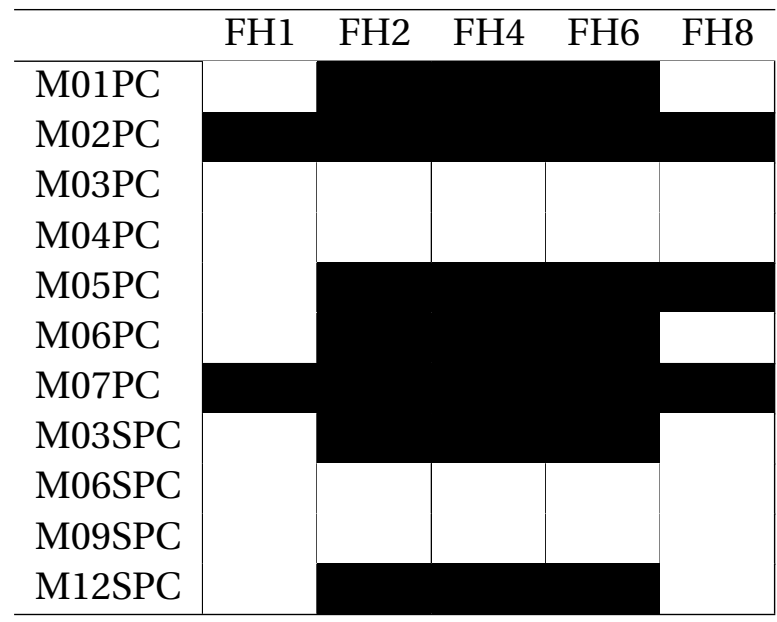

Tabela 5.35 - MCS para LHUR para cada horizonte de previsão quando adicionamos ao caso anterior a possibilidade de modelar as séries por matrizes de covariância após a aplicação de um limiar 
LHUR

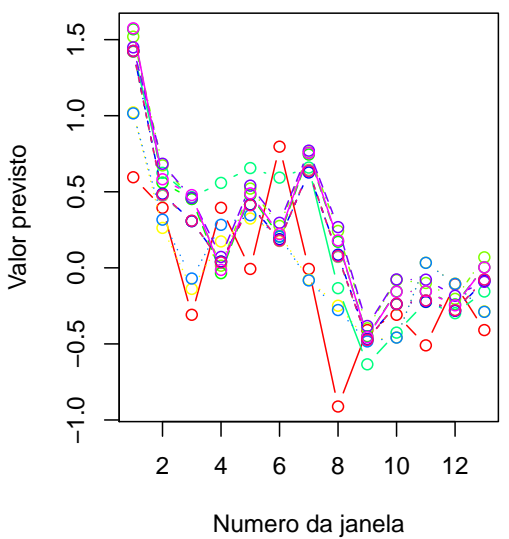

(a) Previsão para 1 trimestre a frente do LHUR

LHUR

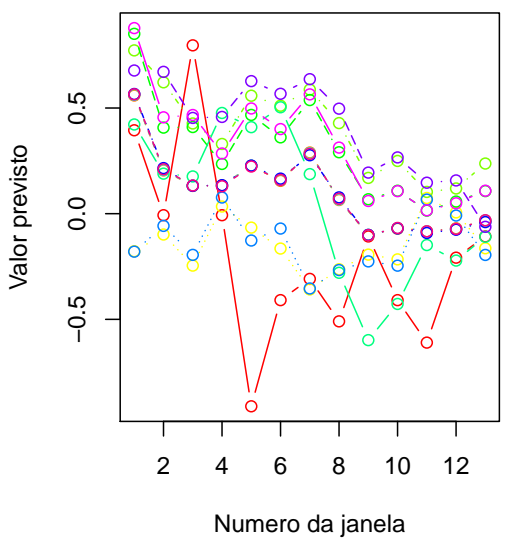

(c) Previsão para 4 trimestres a frente do LHUR
LHUR

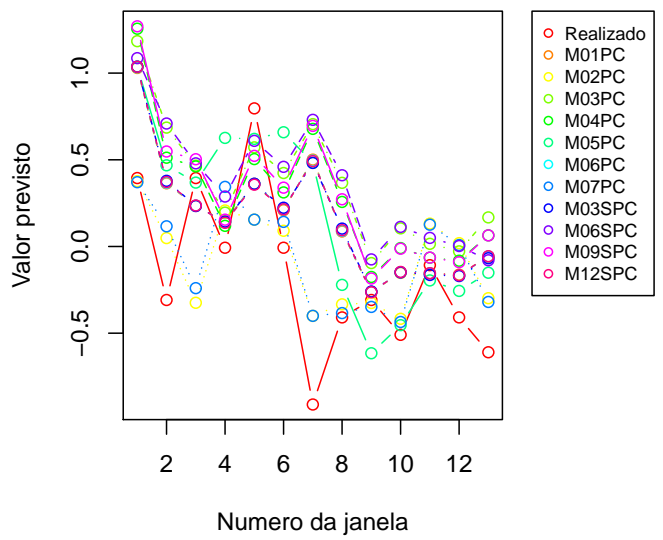

(b) Previsão para 2 trimestres a frente do LHUR

LHUR

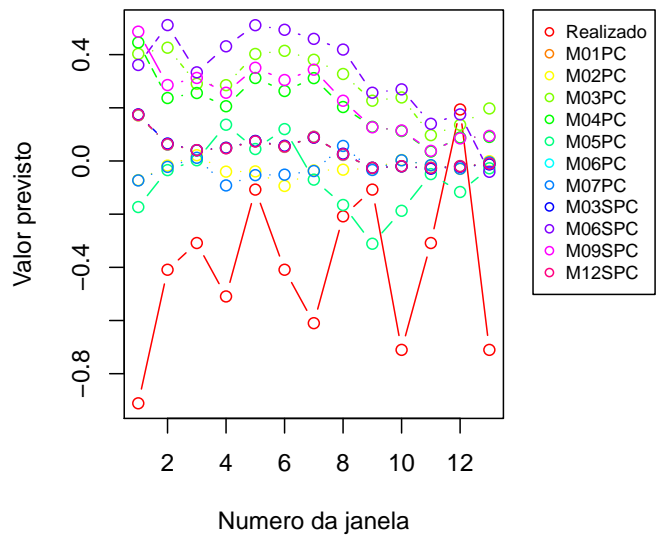

(d) Previsão para 8 trimestres a frente do LHUR

Figura 5.22 - Previsão do LHUR para os próximos 1,2,4 e 8 trimestres considerando os modelos do caso 3. 
LHUR

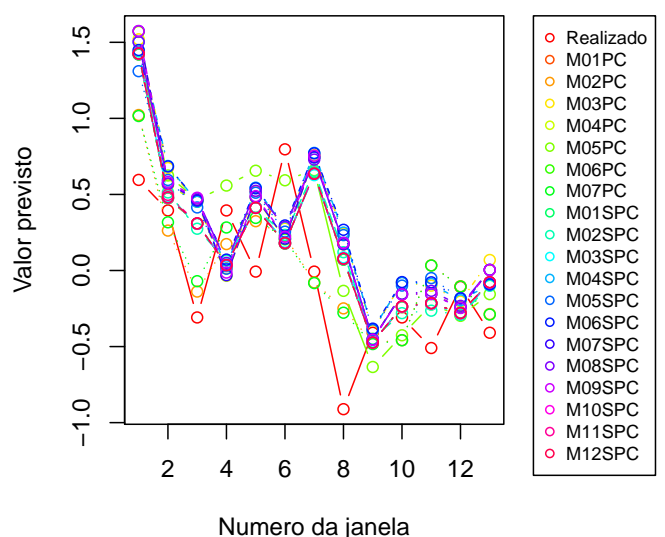

(a) Previsão para 1 trimestre a frente do LHUR

LHUR

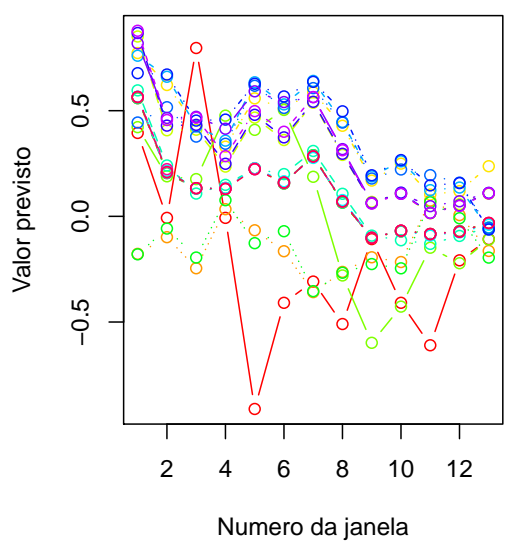

(c) Previsão para 4 trimestres a frente do LHUR
LHUR

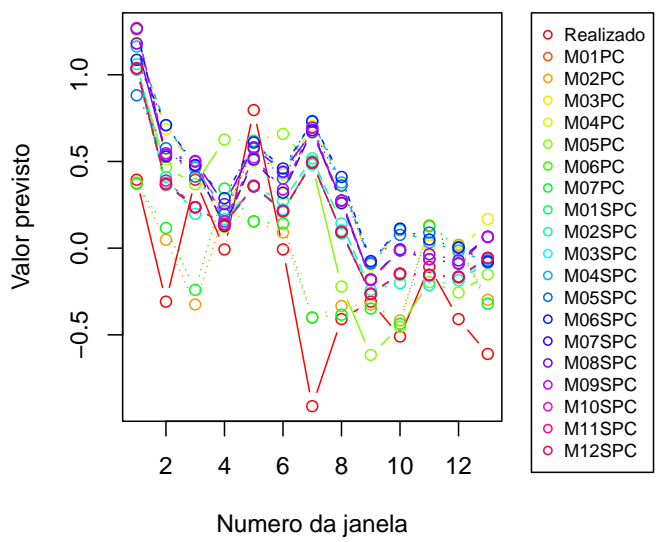

(b) Previsão para 2 trimestres a frente do LHUR

LHUR

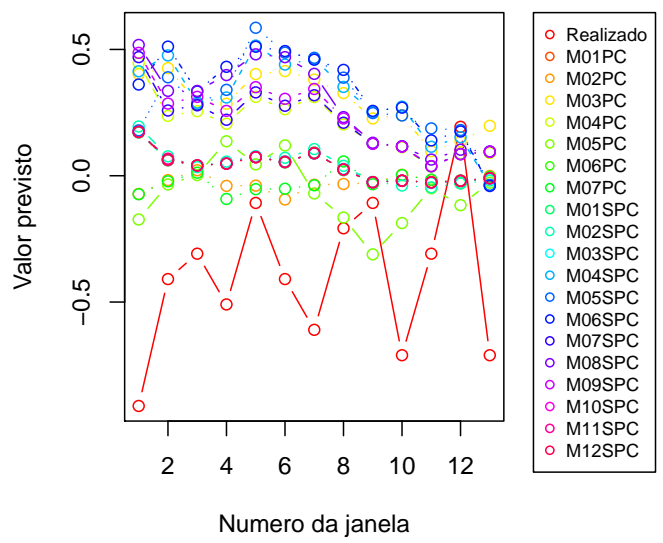

(d) Previsão para 8 trimestres a frente do LHUR

Figura 5.23 - Previsão do LHUR para os próximos 1,2,4 e 8 trimestres considerando os modelos do caso 4.

Ao flexibilizarmos o número de variáveis que constituem os fatores, temos as previsões apresentadas na Figura 5.23.

A Tabela 5.34 indica que essa flexibilização não altera os modelos com menor MSFE em cada horizonte de previsão. Porém, a Tabela 5.35 indica que alguns dos modelos obtidos por essa flexibilização, como o M01SPC e M02SPC, estão presente no MCS em múltiplos horizontes preditivos.

Assim como para GDP251 e IPS10, consideramos também as previsões realizadas pelos modelos de regressão com penalidade e pelo GDFM com o número de choques comuns estimado pelo critério apresentado em Hallin, Liska et al. (2007). Tais previsões são 


\begin{tabular}{cccccc}
\hline & FH1 & FH2 & FH4 & FH6 & FH8 \\
\hline LHUR & M07PC & M07PC & M07PC & M02PC & M05PC \\
\hline
\end{tabular}

Tabela 5.36 - Modelo para LHUR com o menor MSFE para cada horizonte de previsão quando consideramos as matrizes de covariância amostral, Andrews, Fixed-B, matriz após a aplicação de um limiar e o método de extração pode ser PCA ou SPCA.

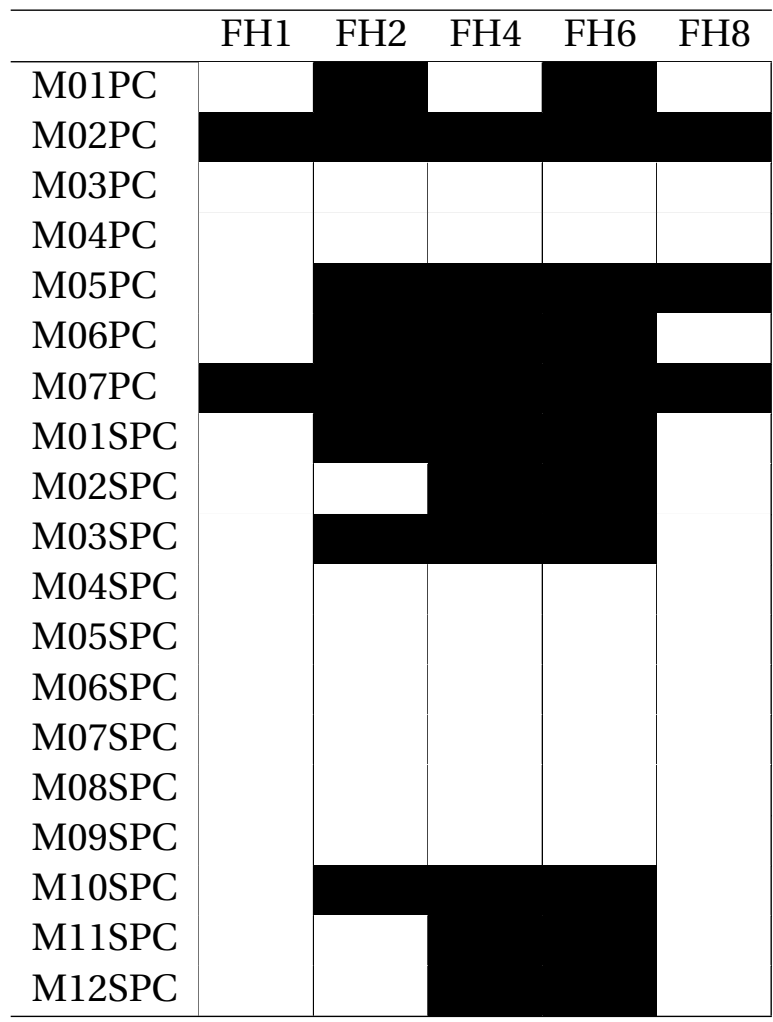

Tabela 5.37 - MCS para LHUR para cada horizonte de previsão quando consideramos as matrizes de covariância amostral, Andrews, Fixed-B, matriz após a aplicação de um limiar e o método de extração pode ser PCA ou SPCA.

representadas pela Figura 5.24.

De acordo com a Tabela 5.38, diferentemente do caso para GDP251 e IPS10, o GDFM não possui o menor MSFE para nenhum dos horizontes analisados. Mas, este modelo esta presente no MCS para 4 dos 5 horizontes de previsão considerados. Ao analisarmos a Tabela 5.39, nota-se que para LHUR, apenas dois modelos, M02PC e M07PC, estão presentes no MCS para previsões relativas ao próximo trimestre. Tal situação é contrasta com os resultados obtidos para GDP251 e IPS10 em que muitos modelos estavam presentes para horizontes curtos e um quantidade menor em horizontes mais longos.

\begin{tabular}{cccccc}
\hline & FH1 & FH2 & FH4 & FH6 & FH8 \\
\hline LHUR & M07PC & M07PC & M07PC & M02PC & M05PC \\
\hline
\end{tabular}

Tabela 5.38 - Modelo para LHUR com o menor MSFE para cada horizonte de previsão quando consideramos todos os modelos apresentados na seção anterior. 
LHUR

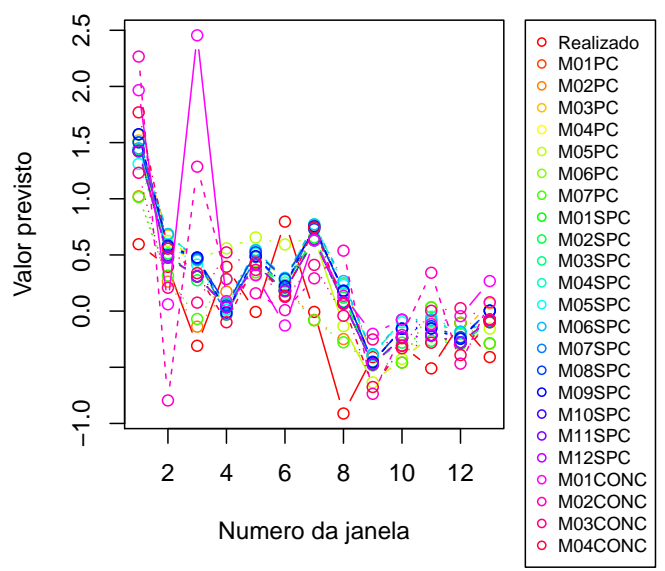

(a) Previsão para 1 trimestre a frente do LHUR

LHUR

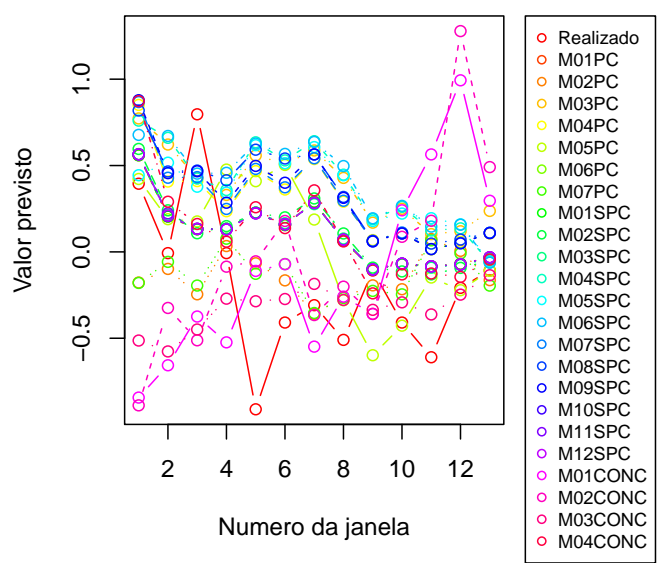

(c) Previsão para 4 trimestres a frente do LHUR
LHUR

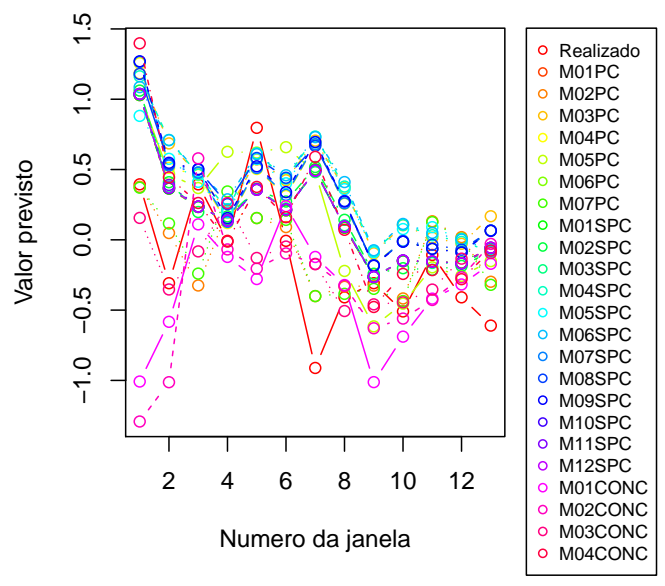

(b) Previsão para 2 trimestres a frente do LHUR

LHUR

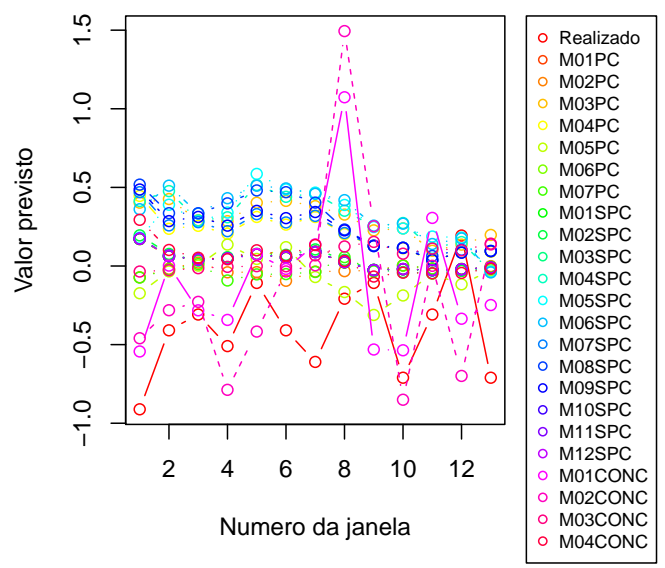

(d) Previsão para 8 trimestres a frente do LHUR

Figura 5.24 - Previsão do LHUR para os próximos 1,2,4 e 8 trimestres considerando os modelos do caso 5 . 


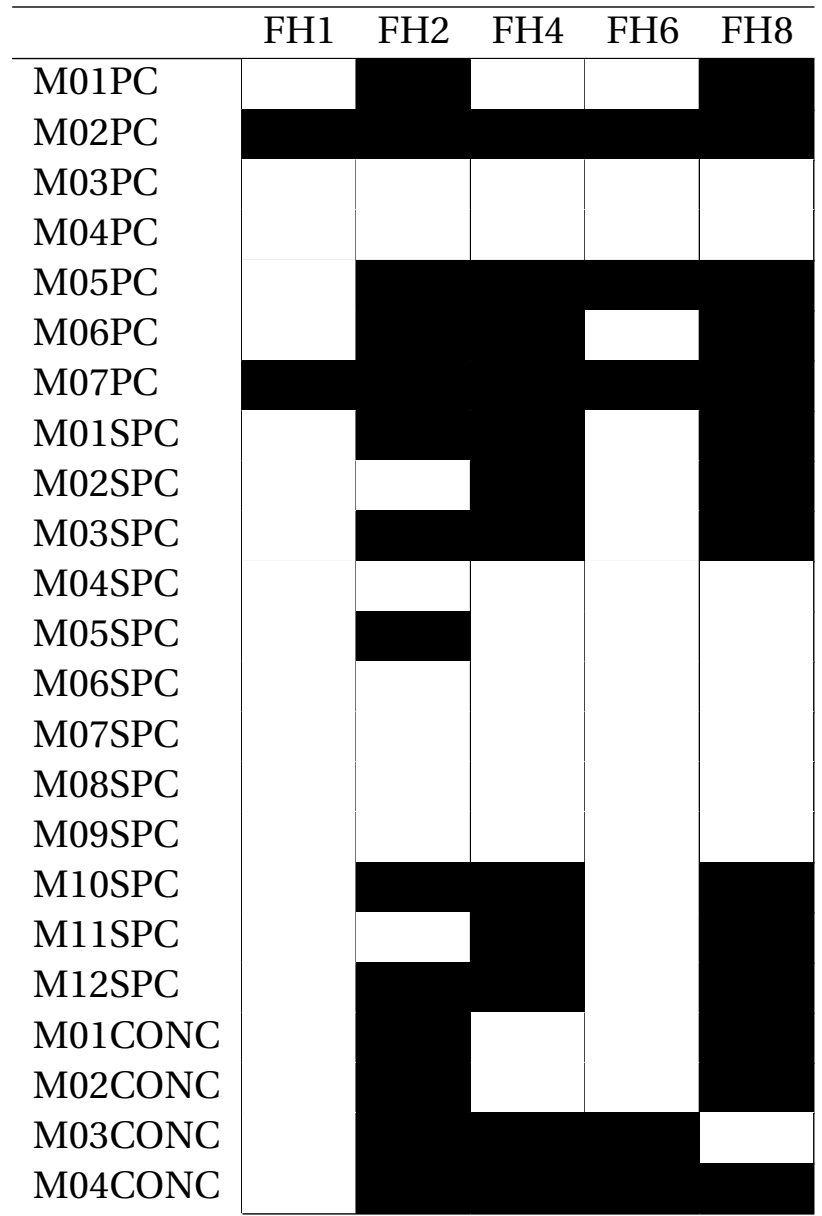

Tabela 5.39 - MCS para LHUR para cada horizonte de previsão quando consideramos todos os modelos apresentados na seção anterior. 
CPIAUCSL

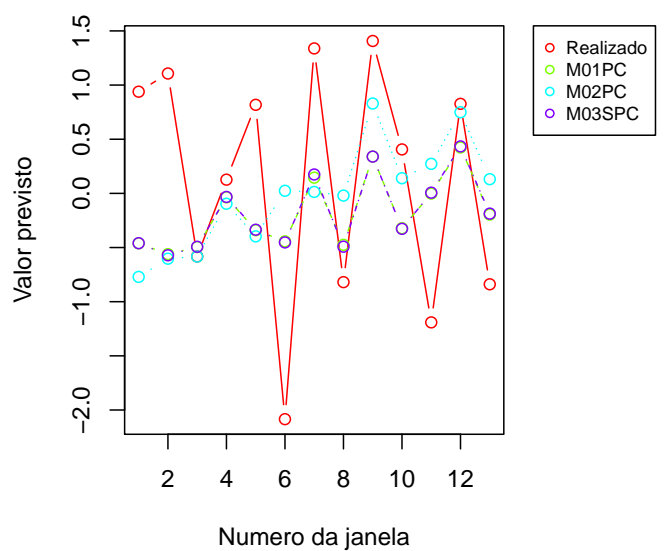

(a) Previsão para 1 trimestre a frente do CPIAUCSL

CPIAUCSL

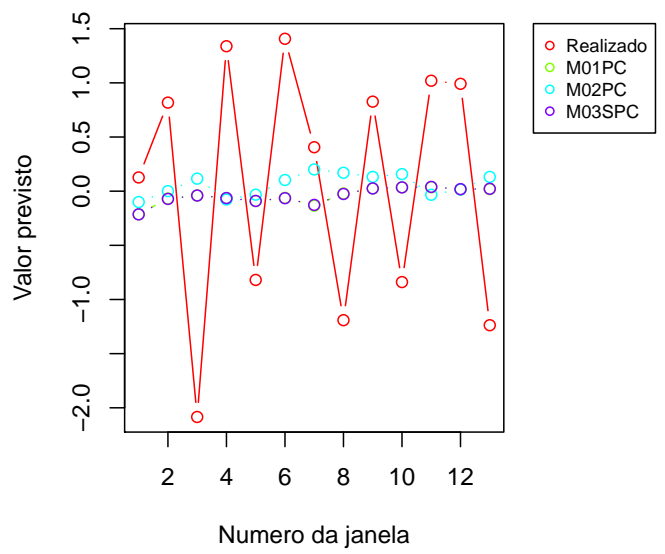

(c) Previsão para 4 trimestres a frente do CPIAUCSL
CPIAUCSL

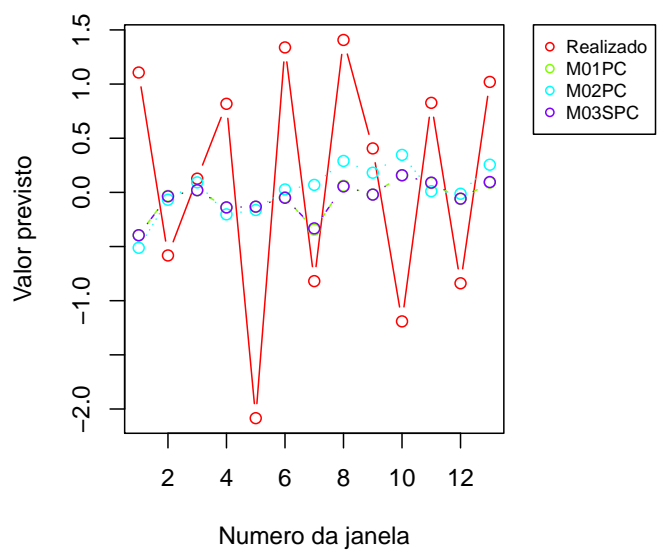

(b) Previsão para 2 trimestres a frente do CPIAUCSL

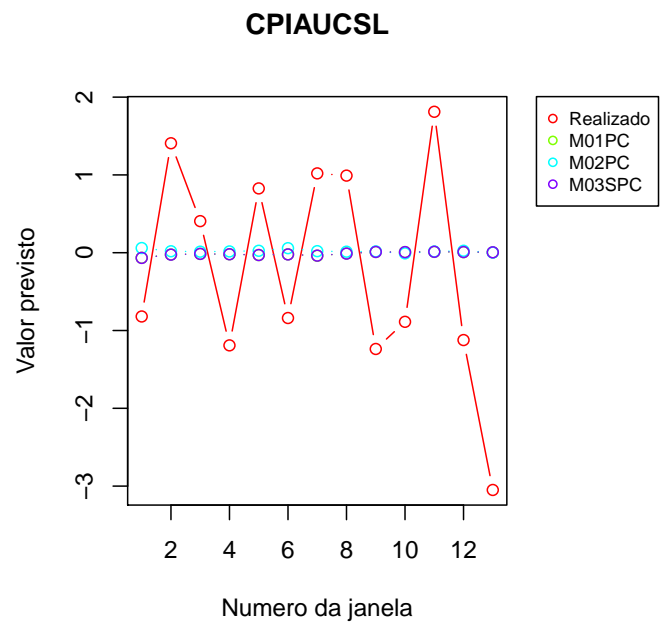

(d) Previsão para 8 trimestres a frente do CPIAUCSL

Figura 5.25 - Previsão do CPIAUCSL para os próximos 1,2,4 e 8 trimestres considerando os modelos do caso 1.

\subsubsection{CPIAUCSL}

Similarmente as variáveis analisadas anteriormente, começamos considerando apenas os modelos baseados na matriz de covariância amostral estimados por PCA ou pelo SPCA com número fixo de variáveis na construção dos fatores.

Variáveis relacionadas a nível de preço/inflação são, tipicamente, consideradas como difíceis de serem previstas e, ao observar as previsões feitas na Figura 5.25 verificamos essa dificuldade.

Os modelos com menor MSFE são apresentados na Tabela 5.40. Note que o modelo 
M03SPC é classificado apresenta o menor MSFE para a maior parte dos horizontes de previsão. Mas, o MCS, apresentado na Tabela 5.41, não consegue diferenciar os modelos em quase nenhum caso. O único modelo que é excluído do MCS é M02PC para FH1. Lembre-se que o MCS apenas testa sequencialmente se os modelos possuem EPA, mas não informa se tais previsões são de fato próximas ao realizado.

\begin{tabular}{lccccc}
\hline & FH1 & FH2 & FH4 & FH6 & FH8 \\
\hline CPIAUCSL & M03SPC & M01PC & M03SPC & M03SPC & M01PC \\
\hline
\end{tabular}

Tabela 5.40 - Modelos para CPIAUCSL com o menor MSFE para cada horizonte de previsão quando consideramos apenas os modelos que usam a matriz de covariância amostral e o método de extração é PCA ou SPCA com número fixo de variáveis não nulas.

\begin{tabular}{l|lllll}
\hline & FH1 & FH2 & FH4 & FH6 & FH8 \\
\hline M01PC & & & & & \\
M02PC & & & & & \\
M03SPC & & & & & \\
\hline
\end{tabular}

Tabela 5.41 - MCS para CPIAUCSL para cada horizonte de previsão quando consideramos apenas os modelos que usam a matriz de covariância amostral e o método de extração é PCA ou SPCA com número fixo de variáveis não nulas.

Buscando melhorar as previsões obtidas anteriormente, consideramos o caso em que a dependência entre as séries é modelada por LRCMs. Tais previsões são apresentadas na Figura 5.26.

A Tabela 5.42 indica que 2 modelos que usam matrizes do tipo fixed-b apresentam o menor MSFE entre os modelos analisados. No entanto, os resultados do MCS presentes na Tabela 5.43 indicam que quase todos os modelos possuem habilidade preditivas similares para previsões com horizonte de pelos menos dois trimestres.

\begin{tabular}{cccccc}
\hline & FH1 & FH2 & FH4 & FH6 & FH8 \\
\hline CPIAUCSL & M03SPC & M01PC & M03SPC & M05PC & M04PC
\end{tabular}

Tabela 5.42 - Modelos para CPIAUCSL com o menor MSFE para cada horizonte de previsão quando adicionamos a possibilidade de modelar as séries pelas matrizes de Andrews e Fixed-b e o método de extração é PCA ou SPCA com número fixo de variáveis não nulas.

Assim como para as demais variáveis analisadas, outra possibilidade é considerar CMET. Tais previsões são apresentadas na Figura 5.27.

Apesar dessas novas possibilidades, a Tabela 5.44 indica que para 4 horizontes de previsão, os modelos com menor MSFE são os mesmo analisados anteriormente. A única mudança ocorre para FH4, nessa situação o modelo M12SPC substitui M03SPC. 
CPIAUCSL

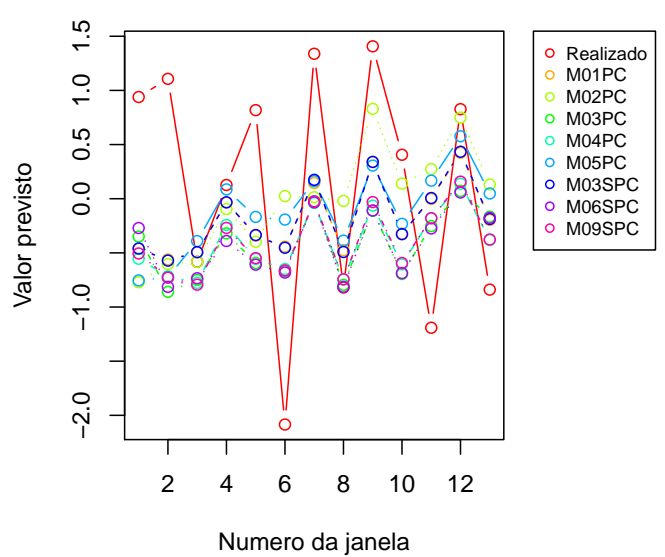

(a) Previsão para 1 trimestre a frente do CPIAUCSL

CPIAUCSL

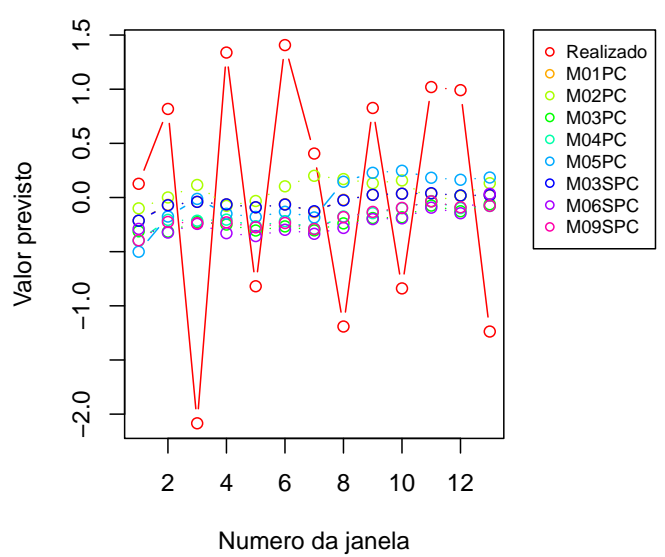

(c) Previsão para 4 trimestres a frente do CPIAUCSL
CPIAUCSL

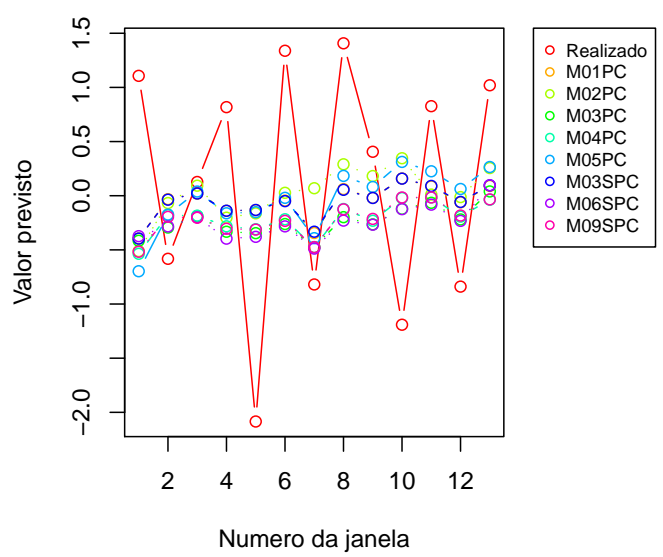

(b) Previsão para 2 trimestres a frente do CPIAUCSL

CPIAUCSL

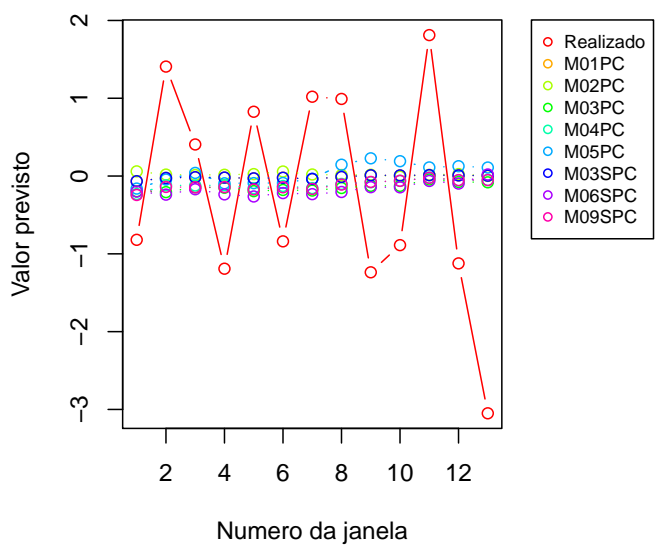

(d) Previsão para 8 trimestres a frente do CPIAUCSL

Figura 5.26 - Previsão do CPIAUCSL para os próximos 1,2,4 e 8 trimestres considerando os modelos do caso 2 . 
CPIAUCSL

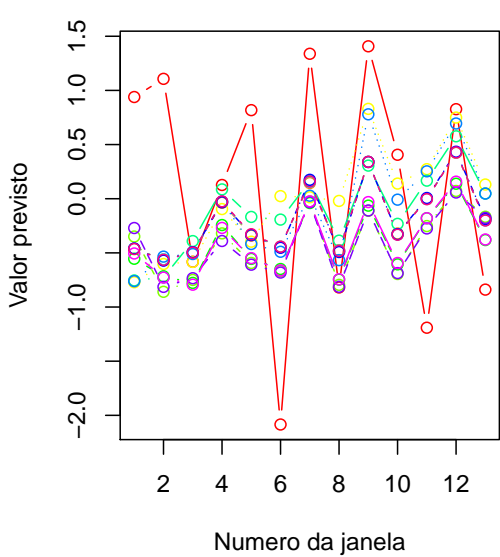

(a) Previsão para 1 trimestre a frente do CPIAUCSL

CPIAUCSL

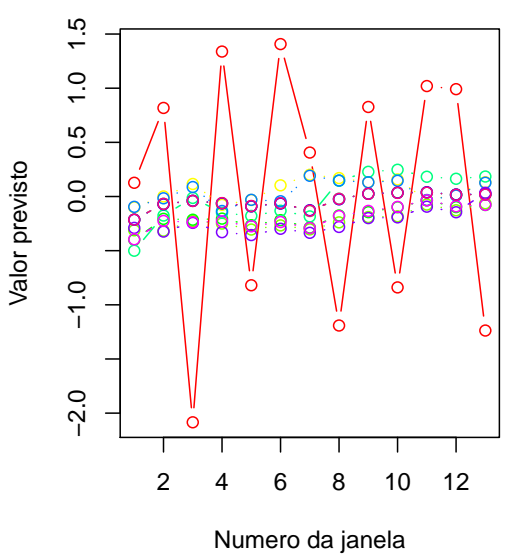

(c) Previsão para 4 trimestres a frente do CPIAUCSL
CPIAUCSL

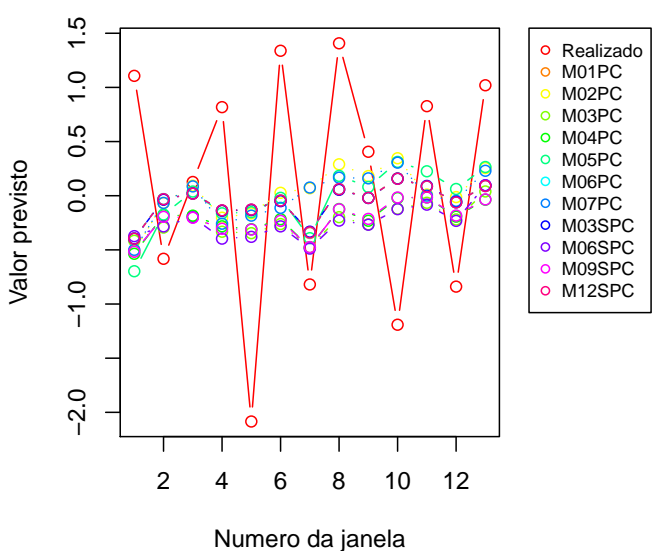

(b) Previsão para 2 trimestres a frente do CPIAUCSL

CPIAUCSL

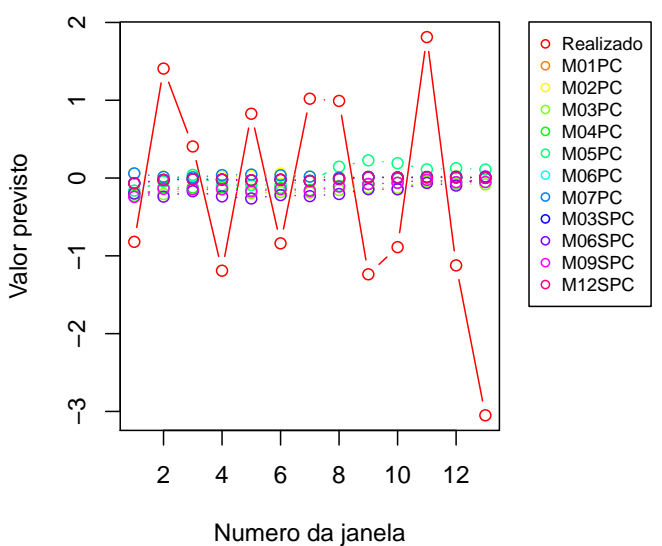

(d) Previsão para 8 trimestres a frente do CPIAUCSL

Figura 5.27 - Previsão do CPIAUCSL para os próximos 1,2,4 e 8 trimestres considerando os modelos do caso 3. 


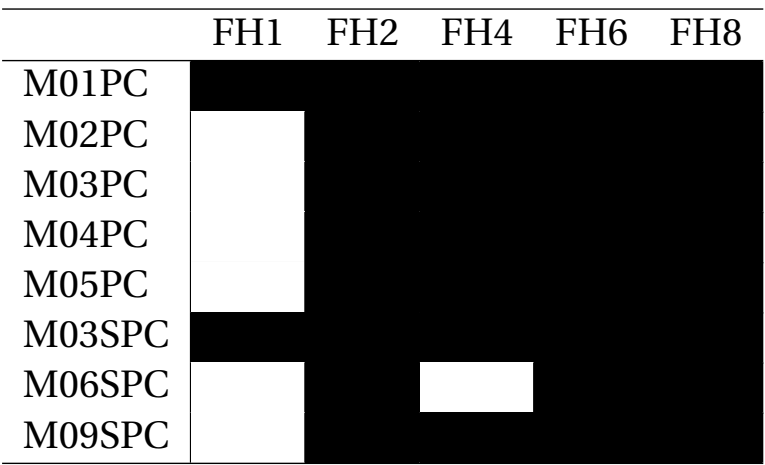

Tabela 5.43 - MCS para CPIAUCSL para cada horizonte de previsão quando adicionamos a possibilidade de modelar as séries pelas matrizes de Andrews e Fixed-b e o método de extração é PCA ou SPCA com número fixo de variáveis não nulas.

Novamente, o MCS consegue rejeitar a hipótese de EPA para poucos casos, especialmente para horizontes de previsão mais distantes, como observado na Tabela 5.45.

\begin{tabular}{cccccc}
\hline & FH1 & FH2 & FH4 & FH6 & FH8 \\
\hline CPIAUCSL & M03SPC & M01PC & M12SPC & M05PC & M04PC \\
\hline
\end{tabular}

Tabela 5.44 - Modelo para CPIAUCSL com o menor MSFE para cada horizonte de previsão quando adicionamos ao caso anterior a possibilidade de modelar as séries por matrizes de covariância após a aplicação de um limiar

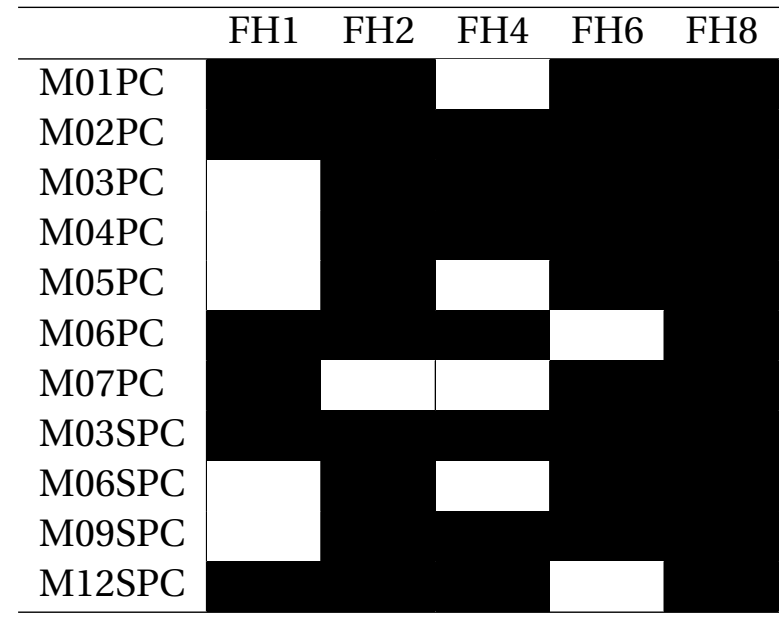

Tabela 5.45 - MCS para CPIAUCSL para cada horizonte de previsão quando adicionamos ao caso anterior a possibilidade de modelar as séries por matrizes de covariância após a aplicação de um limiar

Buscando uma melhora preditiva, consideramos o caso em que os fatores podem ser constituídos por um número distinto de variáveis em cada janela. As previsões nessa situação são representadas pela Figura 5.28

Nesse caso temos a alteração do modelo com menor MSFE para os três horizontes de previsão mais curtos. Agora os modelos com menor MSFE para os próximos 1,2 e 4 trimestres são M02SPC, M01SPC e M10SPC conforme apresentado na Tabela 5.46.O MCS não consegue 
CPIAUCSL

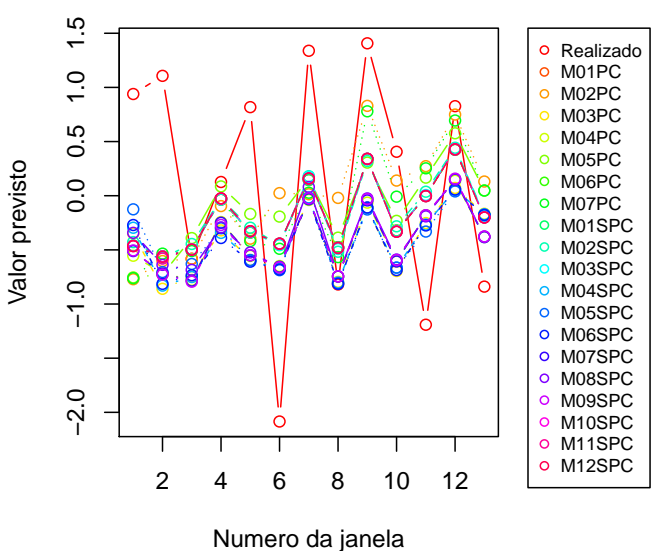

(a) Previsão para 1 trimestre a frente do CPIAUCSL

CPIAUCSL

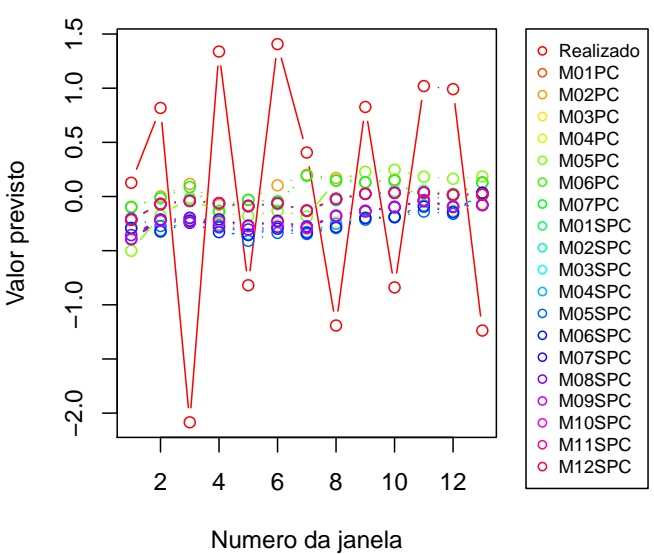

(c) Previsão para 4 trimestres a frente do CPIAUCSL
CPIAUCSL

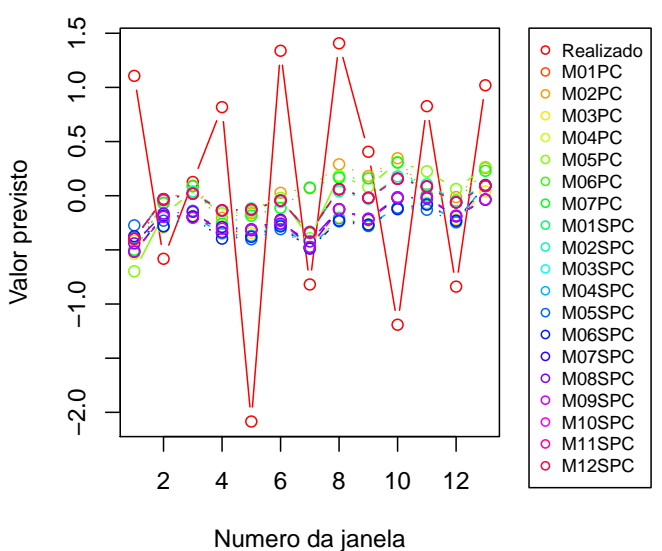

(b) Previsão para 2 trimestres a frente do CPIAUCSL

CPIAUCSL

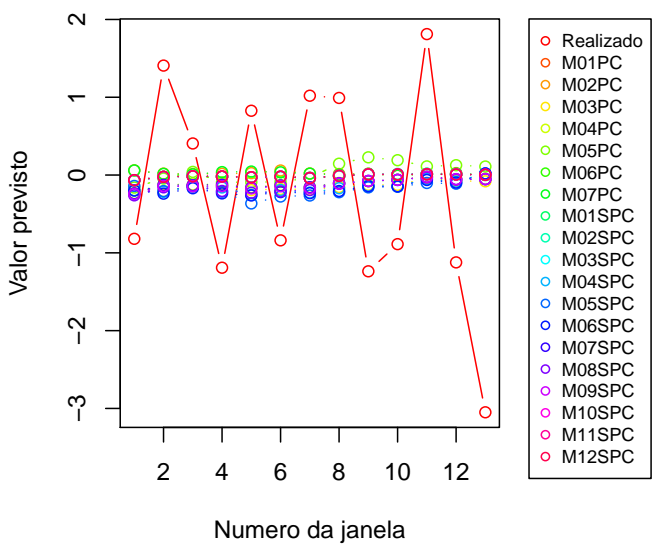

(d) Previsão para 8 trimestres a frente do CPIAUCSL

Figura 5.28 - Previsão do CPIAUCSL para os próximos 1,2,4 e 8 trimestres considerando os modelos do caso 4. 
rejeitar a hipótese nula de EPA para nenhum dos modelos quando consideramos previsões para 8 trimestres à frente como indica a Tabela 5.47 .

\begin{tabular}{cccccc}
\hline & FH1 & FH2 & FH4 & FH6 & FH8 \\
\hline CPIAUCSL & M02SPC & M01SPC & M10SPC & M05PC & M04PC \\
\hline
\end{tabular}

Tabela 5.46 - Modelo para CPIAUCSL com o menor MSFE para cada horizonte de previsão quando consideramos as matrizes de covariância amostral, Andrews, Fixed-B, matriz após a aplicação de um limiar e o método de extração pode ser PCA ou SPCA.

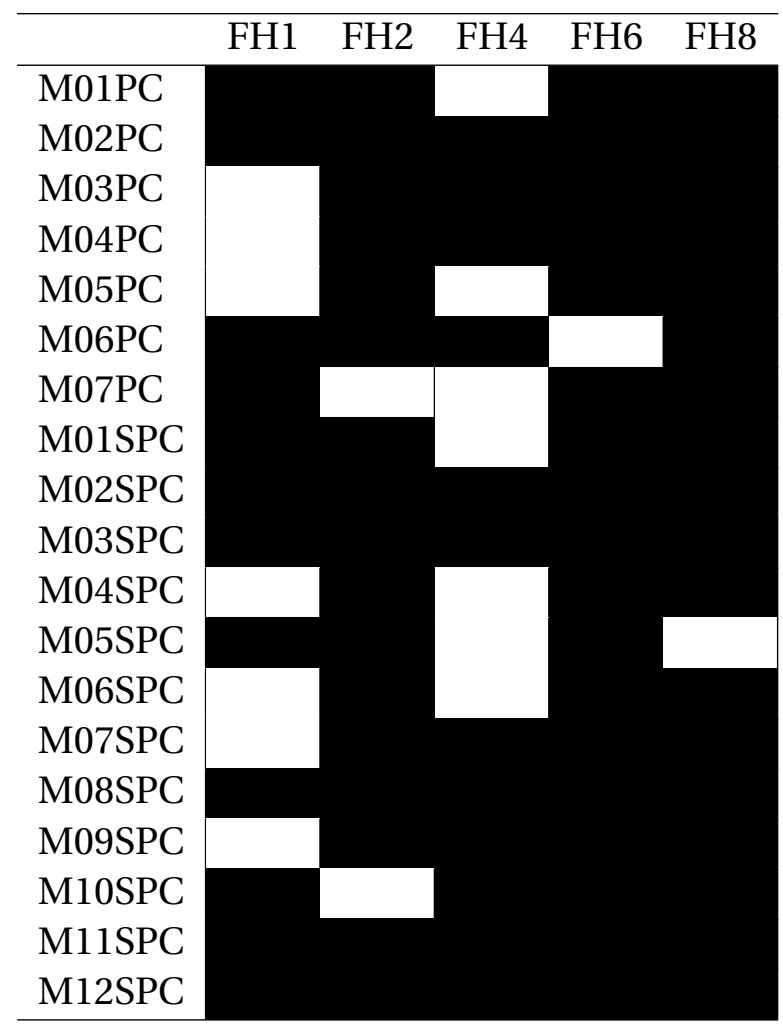

Tabela 5.47 - MCS para CPIAUCSL para cada horizonte de previsão quando consideramos as matrizes de covariância amostral, Andrews, Fixed-B, matriz após a aplicação de um limiar e o método de extração pode ser PCA ou SPCA.

Adicionarmos as previsões dos modelos baseados em regressões com penalização, Figura 5.29, verificamos uma alteração no modelos com menor MSFE para 4 horizontes de previsão. Tais alterações são apresentadas na Tabela 5.46.

\begin{tabular}{cccccc}
\hline & FH1 & FH2 & FH4 & FH6 & FH8 \\
\hline CPIAUCSL & M04CONC & M04CONC & M02CONC & M05PC & M03CONC \\
\hline
\end{tabular}

Tabela 5.48 - Modelo para CPIAUCSL com o menor MSFE para cada horizonte de previsão quando consideramos todos os modelos apresentados na seção anterior.

Assim como no caso do IPS10, o GDFM apresenta um bom desempenho preditivo, em termo de MSFE, quando comparado aos demais modelos. Note também que modelos 
CPIAUCSL

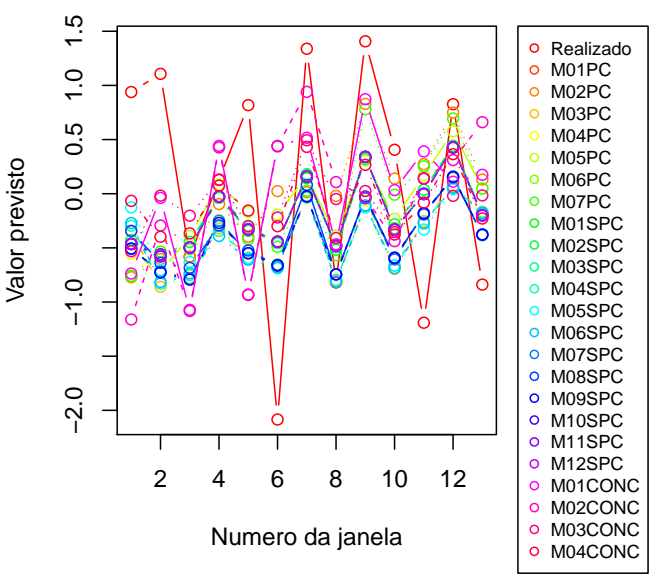

(a) Previsão para 1 trimestre a frente do CPIAUCSL

CPIAUCSL

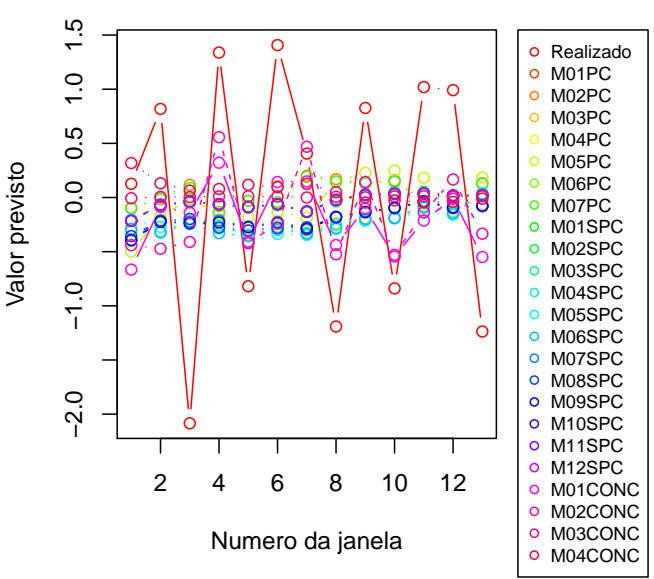

(c) Previsão para 4 trimestres a frente do CPIAUCSL
CPIAUCSL

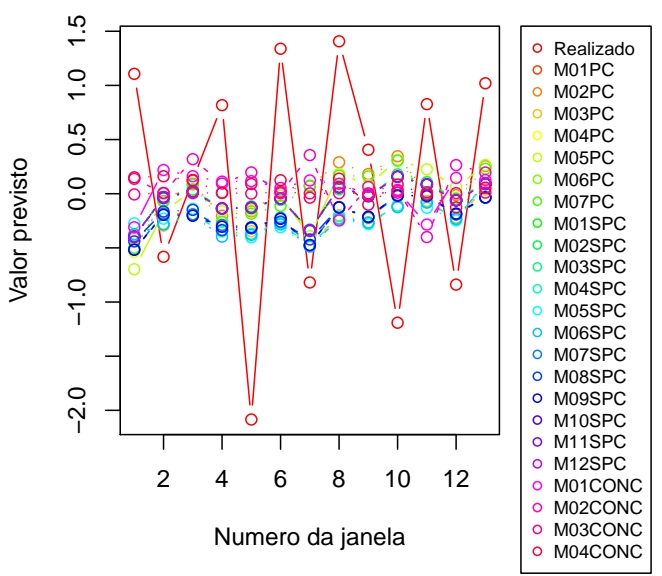

(b) Previsão para 2 trimestres a frente do CPIAUCSL

CPIAUCSL

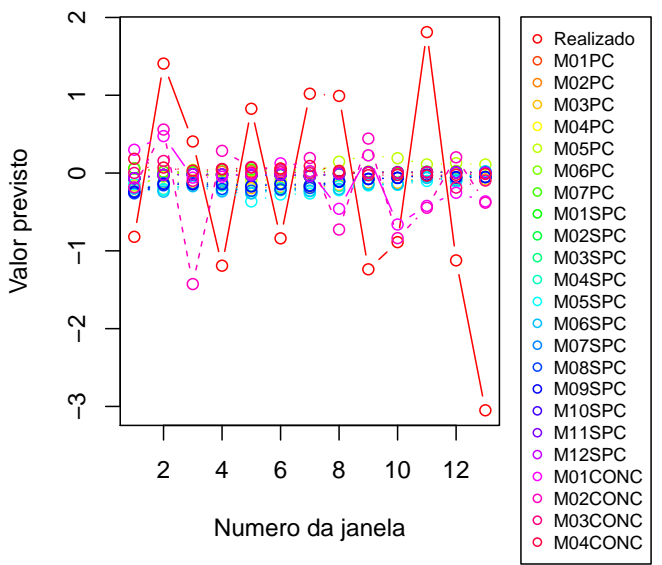

(d) Previsão para 8 trimestres a frente do CPIAUCSL

Figura 5.29 - Previsão do CPIAUCSL para os próximos 1,2,4 e 8 trimestres considerando os modelos do caso 5 . 
baseado no LASSO também possuem um melhor desempenho, em termos de MSFE, do que a maior parte dos modelos fatoriais.

No entanto, como indicado na Tabela 5.49, o MCS não consegue rejeitar H0 para quase nenhum modelo, principalmente para horizontes de previsão maiores. Adicionalmente, a dificuldade em prever variáveis relacionadas ao nível de preço fica clara na Figura 5.29.

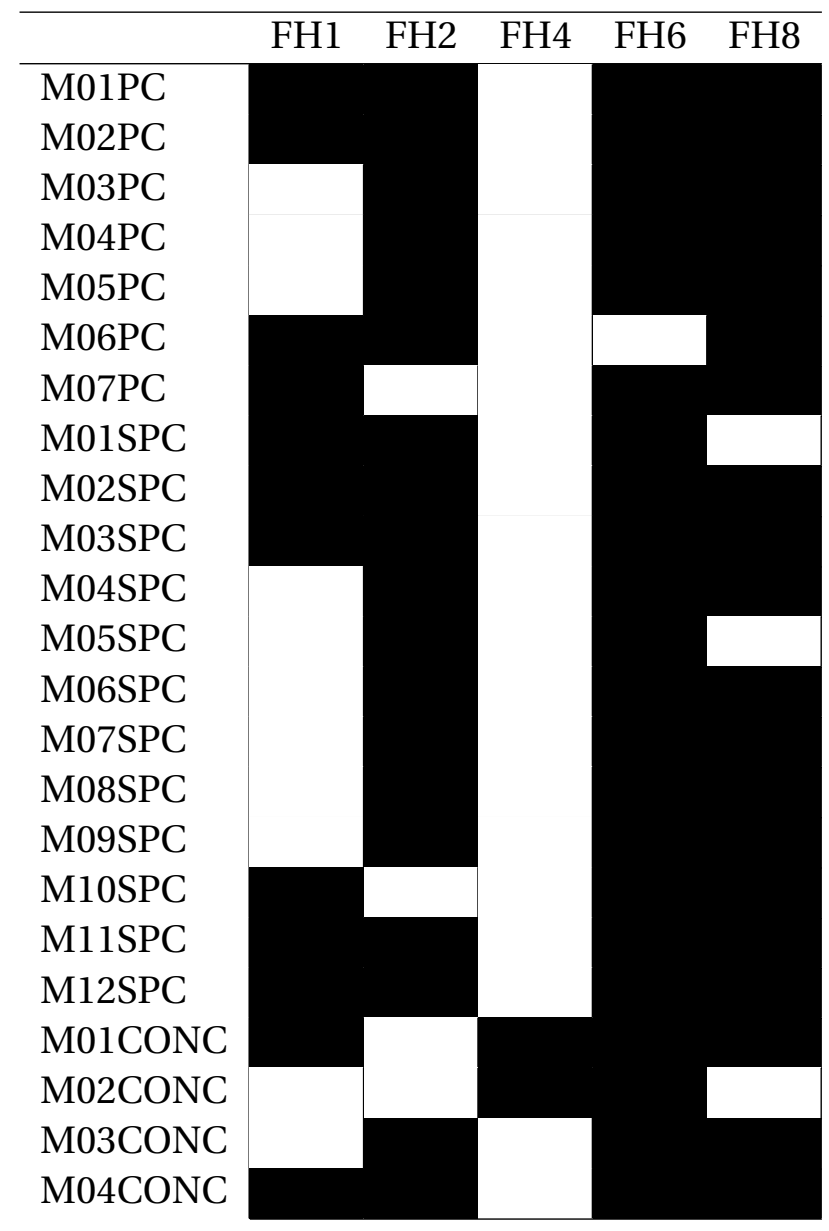

Tabela 5.49 - MCS para CPIAUCSL para cada horizonte de previsão quando consideramos todos os modelos apresentados na seção anterior. 
FSPCOM

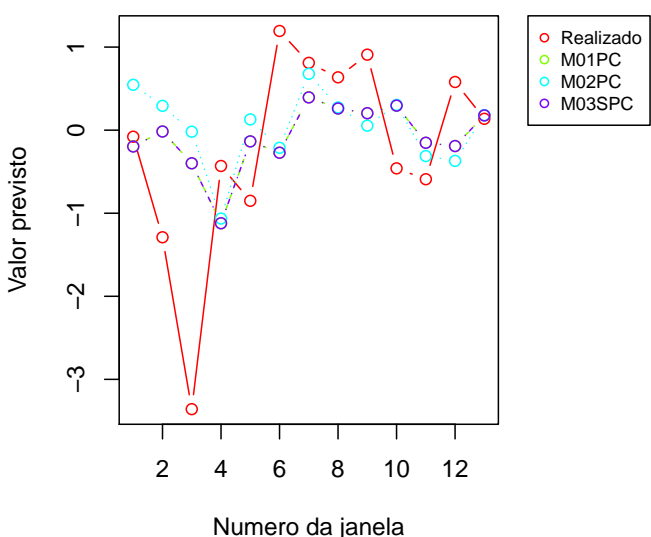

(a) Previsão para 1 trimestre a frente do FSPCOM

FSPCOM

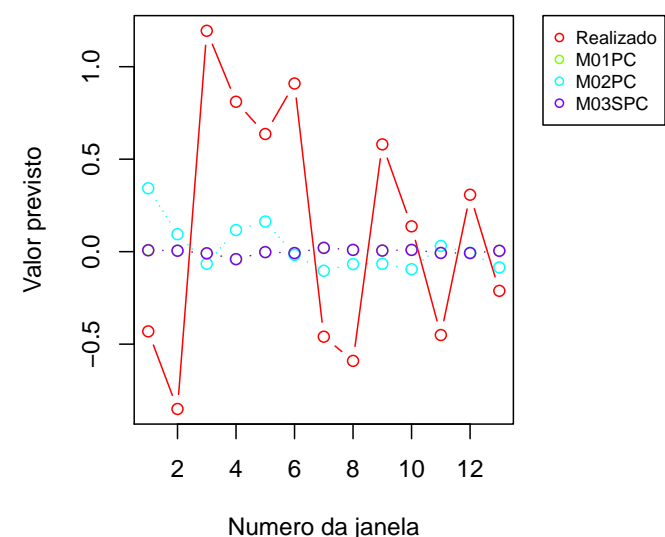

(c) Previsão para 4 trimestres a frente do FSPCOM
FSPCOM

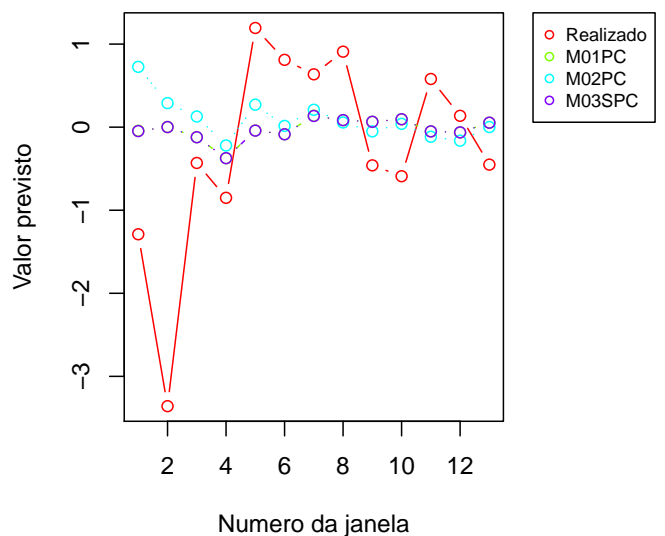

(b) Previsão para 2 trimestres a frente do FSPCOM

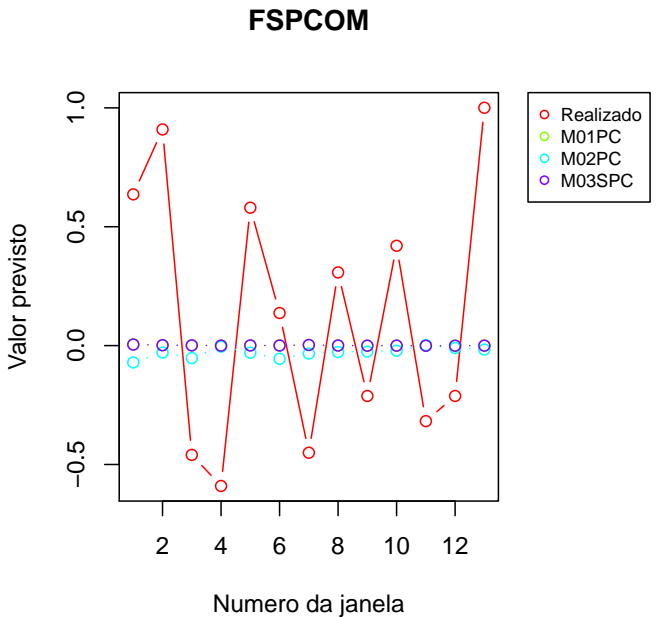

(d) Previsão para 8 trimestres a frente do FSPCOM

Figura 5.30 - Previsão do FSPCOM para os próximos 1,2,4 e 8 trimestres considerando os modelos do caso 1.

\subsubsection{FSPCOM}

Assim como variáveis relacionadas a inflação, variáveis ligadas a índices do mercado acionário são consideradas muito difíceis de serem previstas pela literatura.

Começamos a análise das previsões de FSPCOMP considerando os modelos baseados na matriz de covariância amostral estimados por PCA ou pelo SPCA com número fixo de variáveis na construção dos fatores apresentados anteriormente. As previsões obtidas por esses modelos são apresentadas na Figura 5.30.

Os modelos com menor MSFE e os resultados do MCS são apresentados nas Tabelas 
5.50 e 5.51 , respectivamente.

\begin{tabular}{cccccc}
\hline & FH1 & FH2 & FH4 & FH6 & FH8 \\
\hline FSPCOM & M03SPC & M03SPC & M03SPC & M01PC & M01PC
\end{tabular}

Tabela 5.50 - Modelos para FSPCOM com o menor MSFE para cada horizonte de previsão quando consideramos apenas os modelos que usam a matriz de covariância amostral e o método de extração é PCA ou SPCA com número fixo de variáveis não nulas.

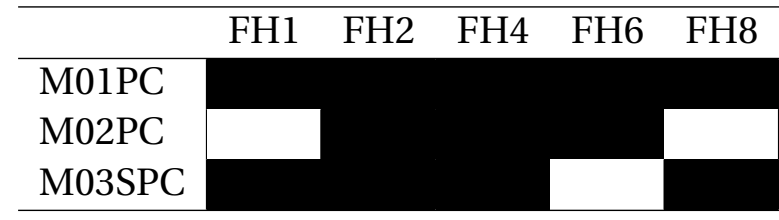

Tabela 5.51 - MCS para FSPCOM para cada horizonte de previsão quando consideramos apenas os modelos que usam a matriz de covariância amostral e o método de extração é PCA ou SPCA com número fixo de variáveis não nulas.

Para FH1 e FH2, os modelos apresentam um desempenho razoável. Porém, a medida que o horizonte de previsão aumenta, os erros tornam-se maiores.

Novamente consideramos modelos que modela a dependência entre as séries por meio de LRCM. As previsões obtidas nessas situações são apresentadas na Figura 5.31.

Como apresentado na Tabela 5.52, M05PC e M09SPC são os modelos com menor MSFE para previsões realizadas para os próximos 6 e 8 trimestres, respectivamente.

Os resultados do MCS, representados na Tabela 5.53, indicam que uma dificuldade em distinguir entre a habilidade preditiva dos modelos para FH2, FH4 e FH8, porém seleciona uma pequena quantidade de modelos para os demais trimestres.

\begin{tabular}{cccccc}
\hline & FH1 & FH2 & FH4 & FH6 & FH8 \\
\hline FSPCOM & M03SPC & M03SPC & M03PC & M05PC & M09SPC \\
\hline
\end{tabular}

Tabela 5.52 - Modelos para FSPCOM com o menor MSFE para cada horizonte de previsão quando adicionamos a possibilidade de modelar as séries pelas matrizes de Andrews e Fixed-b e o método de extração é PCA ou SPCA com número fixo de variáveis não nulas.

Consideramos também a alteração da matriz de covariância amostral por CMET. Tal alteração leva as previsões representadas nas Figuras 5.32.

Note que CMET levam aos melhores modelos, em termos de MSFE, para alguns horizontes. Por exemplo, M07PC e M09SPC são os modelos com menor MSFE para FH4 e FH8 como indicado na Tabela 5.54. A dificuldade do MCS em rejeitar H0 para modelos em horizontes de previsão maiores é similar aos reportados no caso do CPIAUCSL como apresentado na Tabela 5.55. 


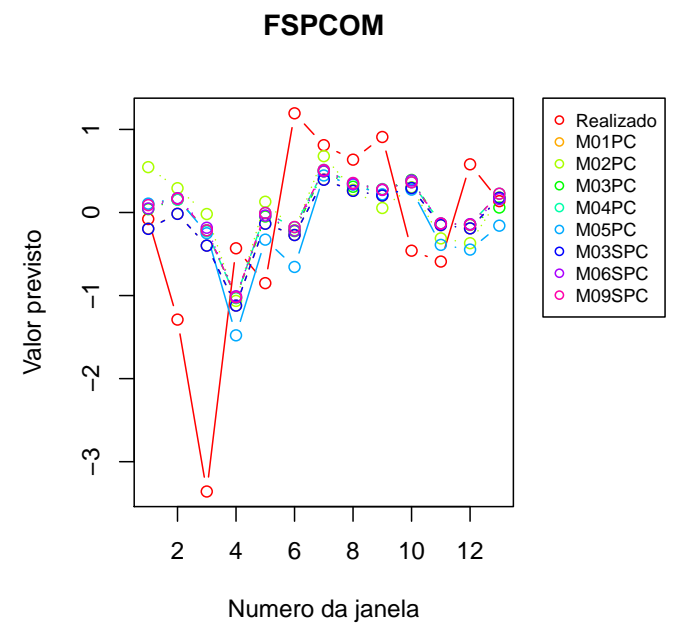

(a) Previsão para 1 trimestre a frente do FSPCOM

FSPCOM

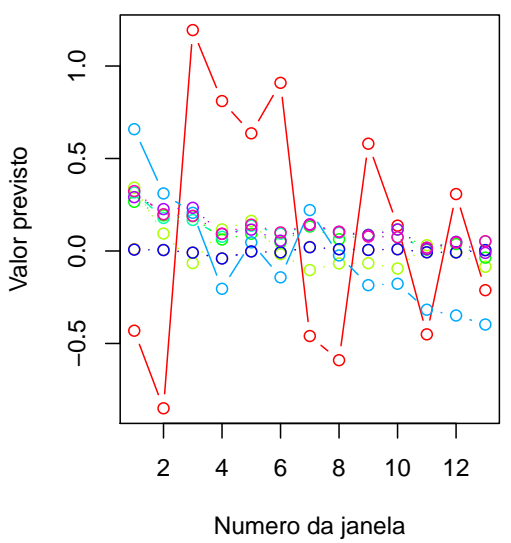

(c) Previsão para 4 trimestres a frente do FSPCOM

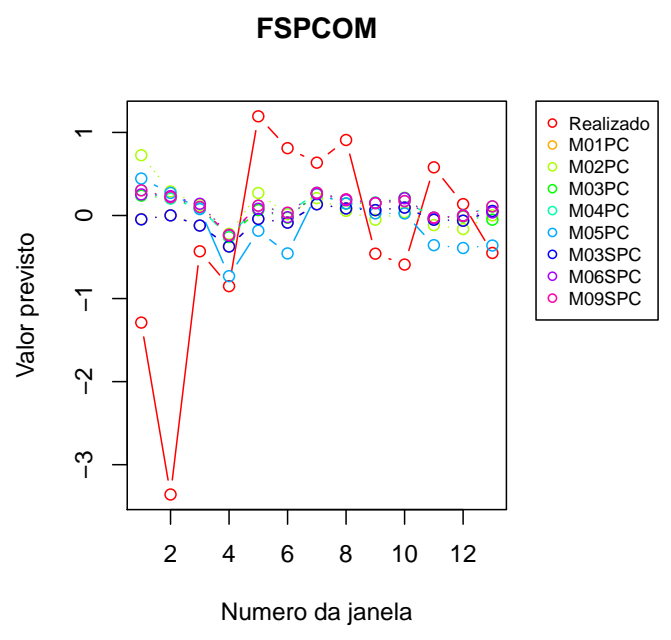

(b) Previsão para 2 trimestres a frente do FSPCOM

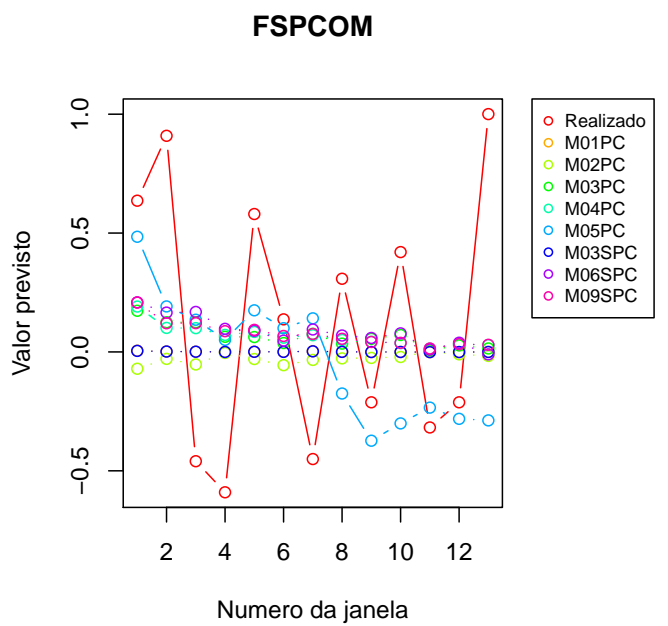

(d) Previsão para 8 trimestres a frente do FSPCOM

Figura 5.31 - Previsão do FSPCOM para os próximos 1,2,4 e 8 trimestres considerando os modelos do caso 2 . 


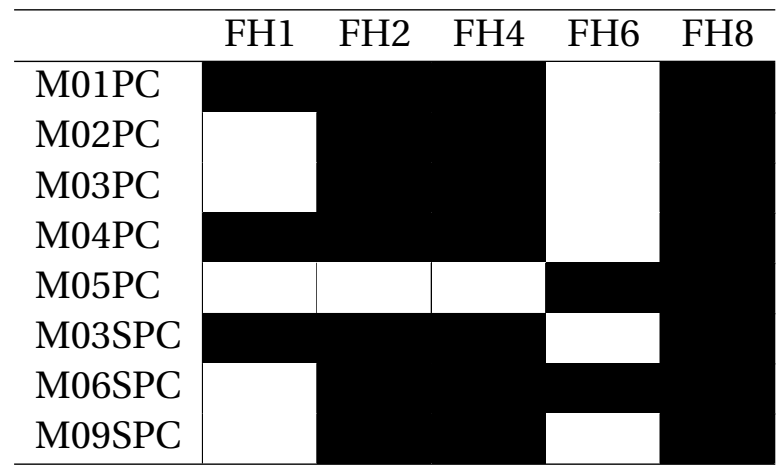

Tabela 5.53 - MCS para FSPCOM para cada horizonte de previsão quando adicionamos a possibilidade de modelar as séries pelas matrizes de Andrews e Fixed-b e o método de extração é PCA ou SPCA com número fixo de variáveis não nulas.

\begin{tabular}{cccccc}
\hline & FH1 & FH2 & FH4 & FH6 & FH8 \\
\hline FSPCOM & M06PC & M03SPC & M07PC & M05PC & M09SPC \\
\hline
\end{tabular}

Tabela 5.54 - Modelo para FSPCOM com o menor MSFE para cada horizonte de previsão quando adicionamos ao caso anterior a possibilidade de modelar as séries por matrizes de covariância após a aplicação de um limiar

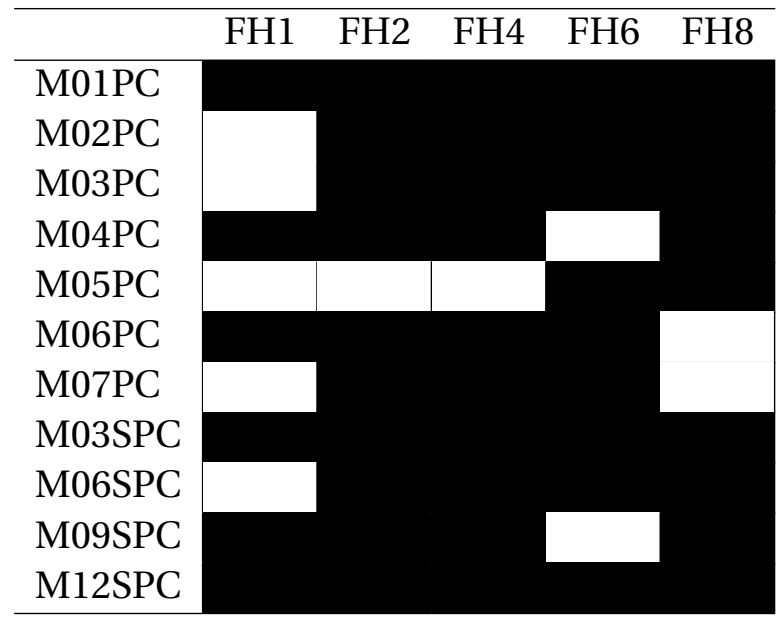

Tabela 5.55 - MCS para FSPCOM para cada horizonte de previsão quando adicionamos ao caso anterior a possibilidade de modelar as séries por matrizes de covariância após a aplicação de um limiar 


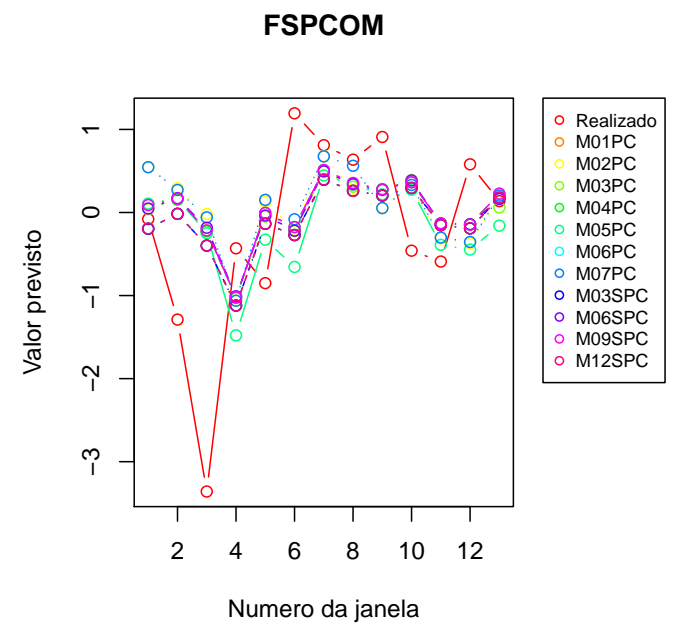

(a) Previsão para 1 trimestre a frente do FSPCOM

FSPCOM

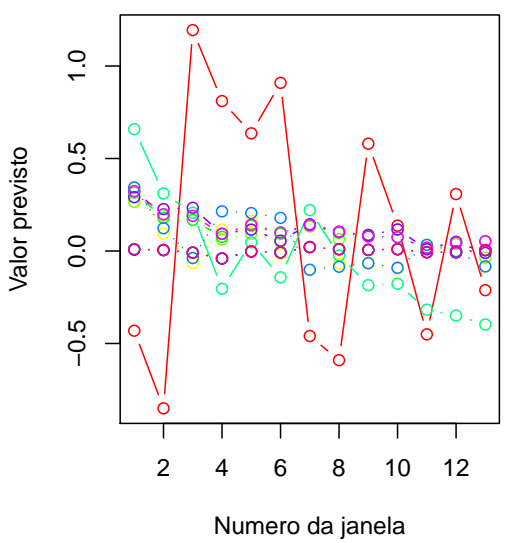

(c) Previsão para 4 trimestres a frente do FSPCOM

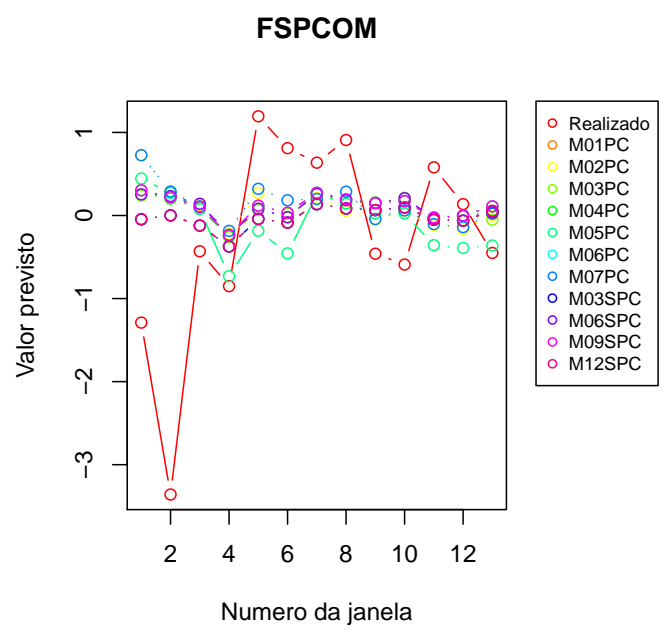

(b) Previsão para 2 trimestres a frente do FSPCOM

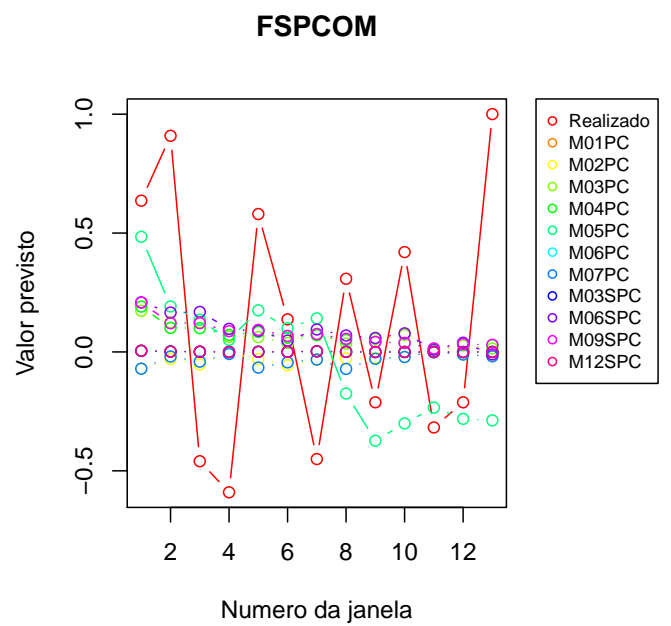

(d) Previsão para 8 trimestres a frente do FSPCOM

Figura 5.32 - Previsão do FSPCOM para os próximos 1,2,4 e 8 trimestres considerando os modelos do caso 3. 


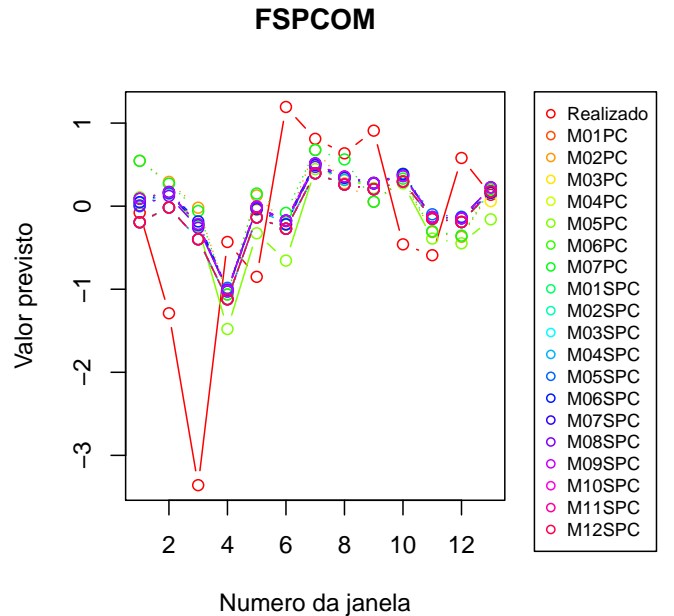

(a) Previsão para 1 trimestre a frente do FSPCOM

FSPCOM

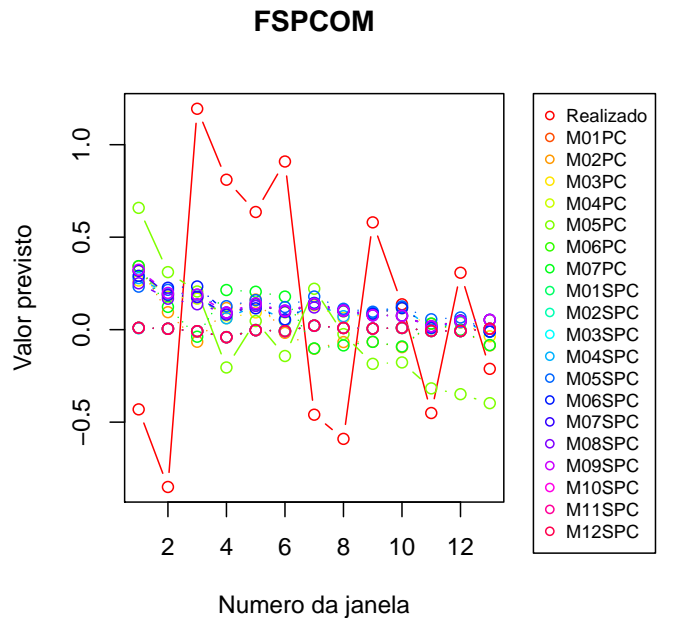

(c) Previsão para 4 trimestres a frente do FSPCOM

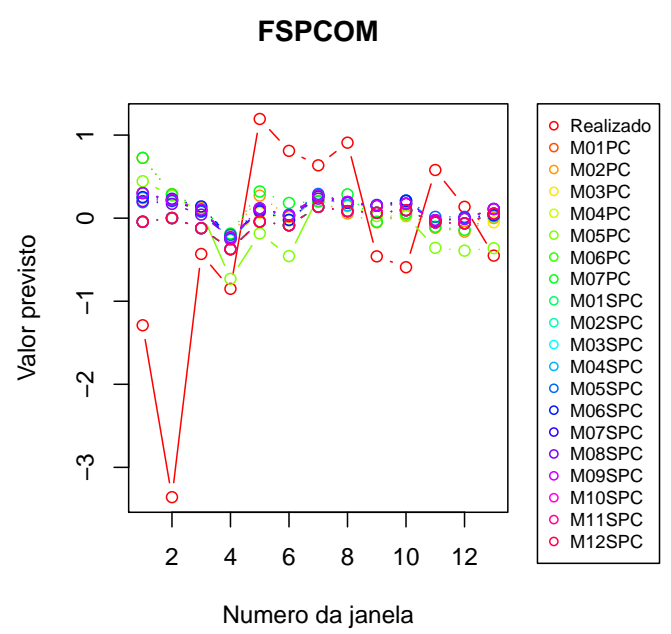

(b) Previsão para 2 trimestres a frente do FSPCOM
Figura 5.33 - Previsão do FSPCOM para os próximos 1,2,4 e 8 trimestres considerando os modelos do caso 4.

Ao permitir que o número de variáveis usadas na construção dos fatores se altere, obtivemos as previsões apresentadas na Figura 5.33.

Como apresentado na Tabela 5.56, essa alteração leva a novos modelos com o menor MSFE para FH1 e FH8. Conforme indicado na Tabela 5.57, apesar na alteração em FH8, o MCS não é capaz de rejeitar a hipótese de EPA para nenhum dos modelos nesse horizonte de previsão.

Por fim, consideramos as previsões realizadas pelos modelos baseados em adaLASSO, LASSO e GDFM. Tais previsões são representadas pela Figura 5.34. 
FSPCOM

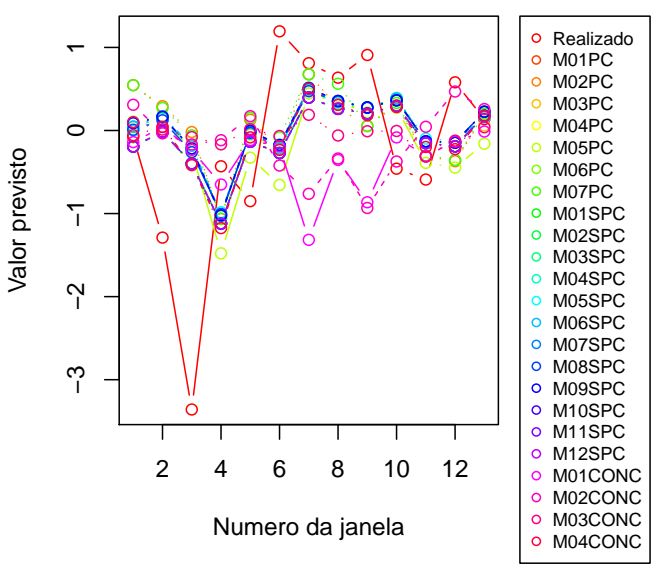

(a) Previsão para 1 trimestre a frente do FSPCOM

FSPCOM

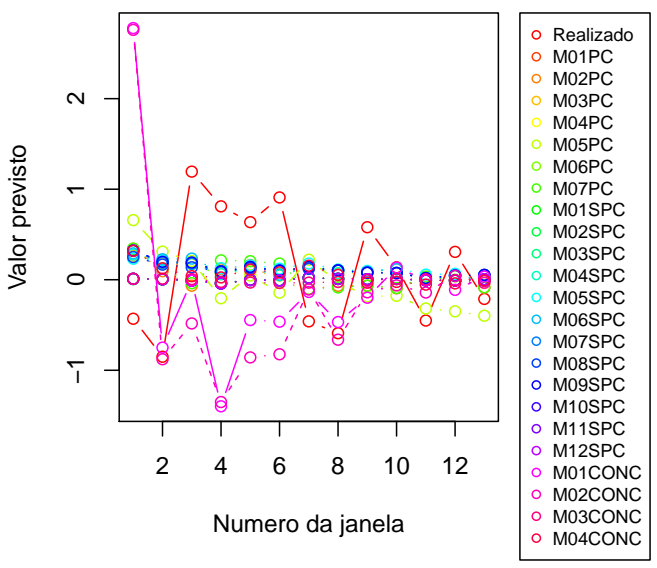

(c) Previsão para 4 trimestres a frente do FSPCOM
FSPCOM

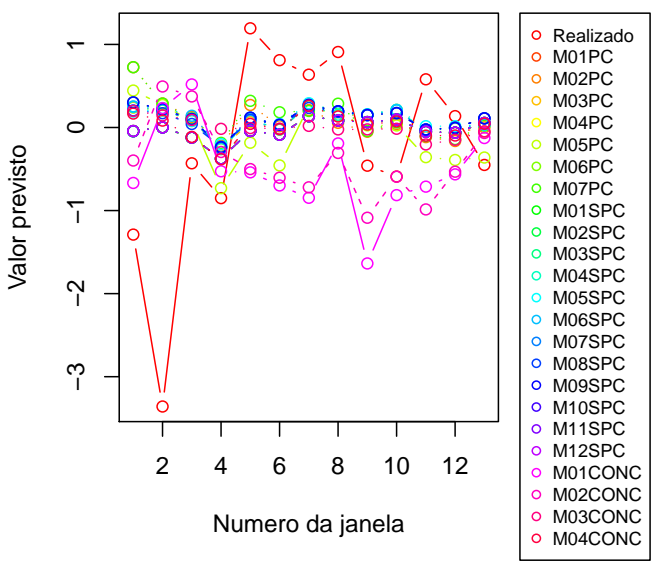

(b) Previsão para 2 trimestres a frente do FSPCOM

FSPCOM

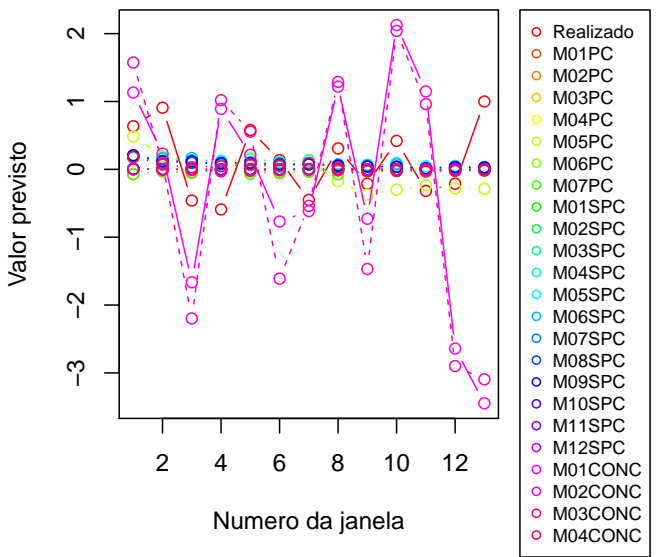

(d) Previsão para 8 trimestres a frente do FSPCOM

Figura 5.34 - Previsão do FSPCOM para os próximos 1,2,4 e 8 trimestres considerando os modelos do caso 5 . 


\begin{tabular}{cccccc}
\hline & FH1 & FH2 & FH4 & FH6 & FH8 \\
\hline FSPCOM & M02SPC & M03SPC & M07PC & M05PC & M08SPC \\
\hline
\end{tabular}

Tabela 5.56 - Modelo para FSPCOM com o menor MSFE para cada horizonte de previsão quando consideramos as matrizes de covariância amostral, Andrews, Fixed-B, matriz após a aplicação de um limiar e o método de extração pode ser PCA ou SPCA.

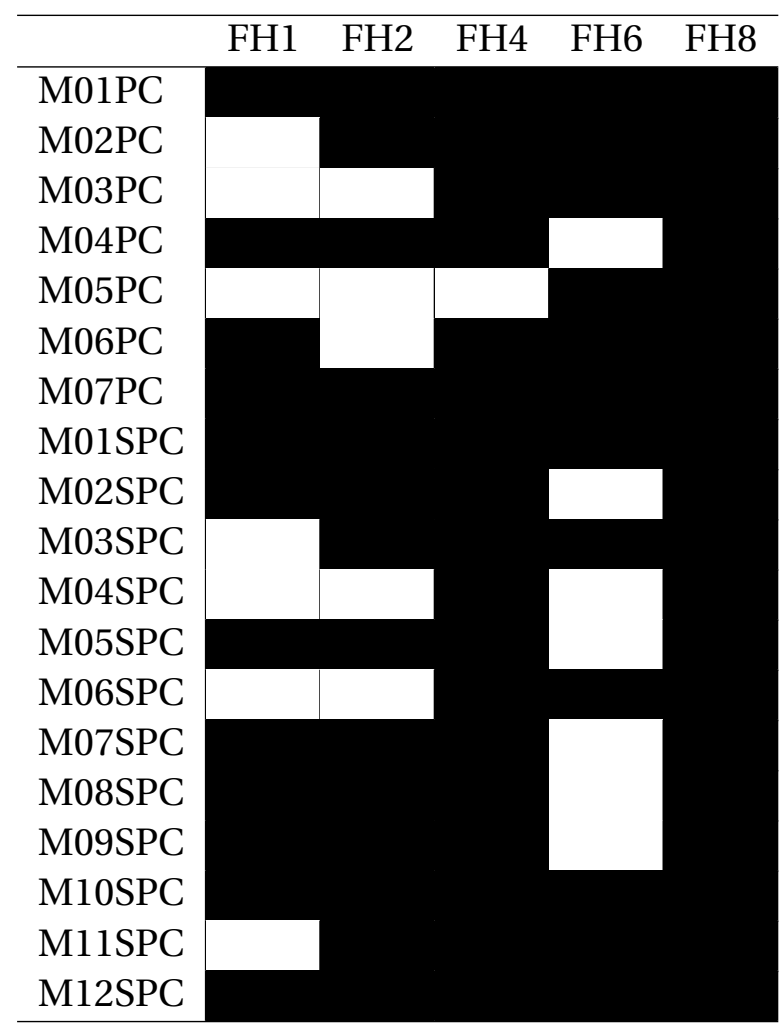

Tabela 5.57 - MCS para FSPCOM para cada horizonte de previsão quando consideramos as matrizes de covariância amostral, Andrews, Fixed-B, matriz após a aplicação de um limiar e o método de extração pode ser PCA ou SPCA.

No entanto, como apresentado na Tabela 5.58, em nenhum horizonte de previsão eles apresentam o menor MSFE. Porém, como indicado na Tabela 5.59, esses modelos estão presente no MCS em diversos horizontes de previsão.

\begin{tabular}{cccccc}
\hline & FH1 & FH2 & FH4 & FH6 & FH8 \\
\hline FSPCOM & M02SPC & M03SPC & M07PC & M05PC & M08SPC \\
\hline
\end{tabular}

Tabela 5.58 - Modelo para FSPCOM com o menor MSFE para cada horizonte de previsão quando consideramos todos os modelos apresentados na seção anterior. 


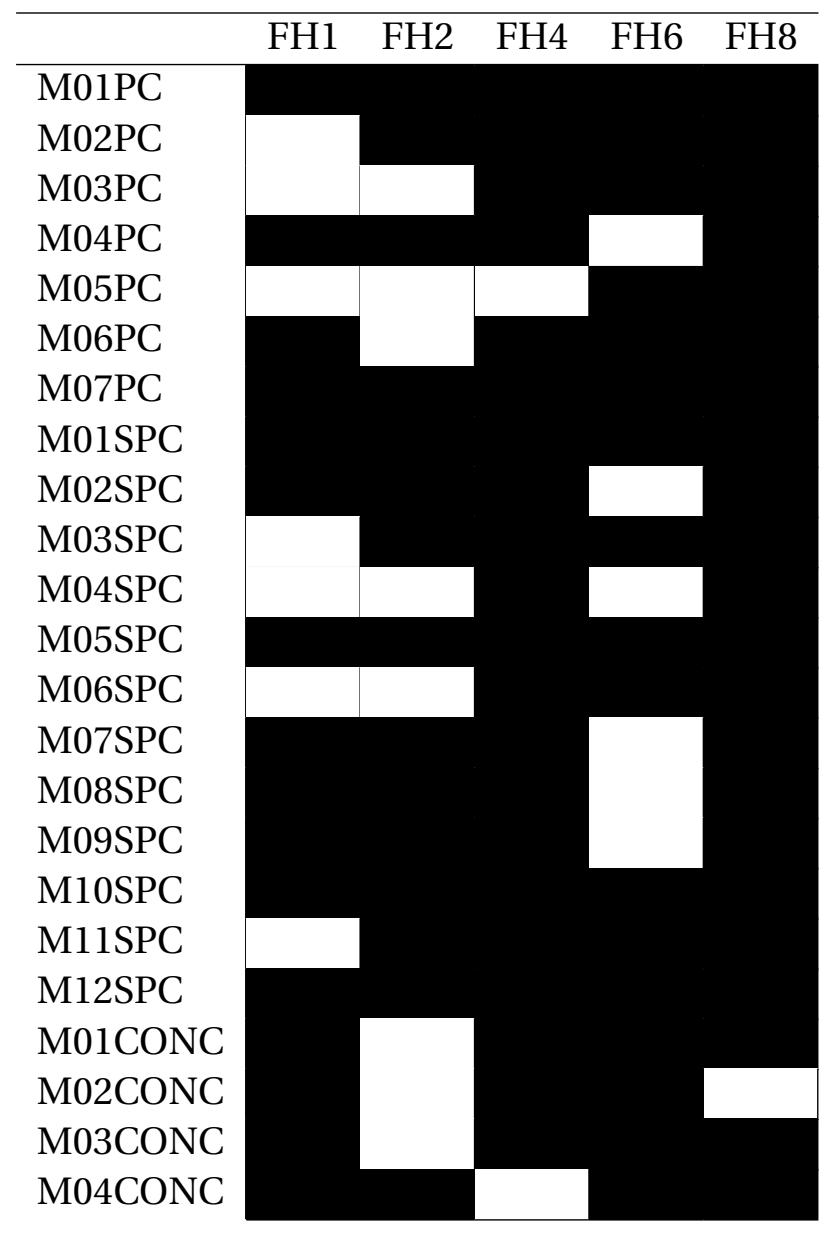

Tabela 5.59 - MCS para FSPCOM para cada horizonte de previsão quando consideramos todos os modelos apresentados na seção anterior. 


\section{CONCLUSÃO}

Neste trabalho, é analisado o impacto preditivo da escolha da matriz de covariância e do método de extração dos fatores latentes em modelos fatoriais.

Tradicionalmente, modelos fatoriais utilizam a matriz de covariância amostral. Nessa situação. A estimação dos fatores usando SPCA leva, em alguns casos, a previsões com menor MSFE do que quando utiliza-se PCA. Esses resultados fortalecem a ideia que a diminuição do número de séries temporais usadas não levam necessariamente a um pior desempenho preditivo assim como concluído por outros autores como, por exemplo, Boivin e Ng (2006). No entanto, na maioria dos casos, o MCS não é capaz de rejeitar a hipótese de mesma habilidade preditiva entre os modelos baseados na matriz de covariância amostral e com fatores construídos por PCA ou SPCA contribuindo com evidência de Kristensen (2017) de que os resultados preditivos obtidos por esse métodos são próximos.

Ao considerar que as séries macroeconômicas podem ser mensuradas com um erro de medida, a diferenciação pode introduzir uma estrutura de dependência temporal. A presença de dependência temporal indica que a matriz de covariância amostral deve ser substituída por uma LRCM.

Uma alteração do conjunto de modelos selecionados pelo MCS quando substituímos a matriz de covariância amostral pela LRCM pode indicar que, na presença de dependência temporal, a seleção de variáveis leva a uma maior robustez a contaminação na matriz de covariância a processos MA. Porém, ao mitigar a dependência temporal pelo uso das matrizes de longo-prazo, modelos que não selecionam as variáveis apresentam um melhor desempenho. A PCA considera que as variáveis são mensuradas sem erro de medida, ao utilizar uma LRCM, essa dependência ao erro de medida é mitigado.

Além da dependência temporal, no contexto de alta dimensão, a matriz de covariância amostral pode acumular erros de estimação devido ao número elevado de parâmetros a serem estimados. Ao usar um hard-threshold, podemos mitigar esse problema. Por exemplo, para a previsão do ISP10 e LHUR, a CMET apresenta um bom desempenho.

Esses resultados expandem a evidência apresentada em Smeekes e Wijler (2018) de que modelos que utilizam esparsidade possuem melhor desempenho do que modelos fatoriais baseados na matriz de covariância amostral e PCA quando temos a presença de erros de especificação.

Adicionalmente, consideramos modelos de regressão com penalização, como o LASSO e adaLASSO, e o GDFM. Ambos os métodos estão presentes no conjunto de habilidade preditiva superior para diversas variáveis em múltiplos horizontes de previsão. Em especial, o GDFM apresenta bom desempenho na previsão do IPS10 em horizontes curtos e para o 
GDP251 em múltiplos horizontes. 


\section{REFERÊNCIAS}

AHN, S. C.; HORENSTEIN, A. R. Eigenvalue ratio test for the number of factors. Econometrica, Wiley Online Library, v. 81, n. 3, p. 1203-1227, 2013.

AIOLFI, M.; CAPISTRÁN, C.; TIMMERMANN, A. Forecast combinations. CREATES Research Paper, n. 2010-21, 2010.

ANDREWS, D. Heteroskedasticity and autocorrelation consistent covariant matrix estimation. Econometrica, v. 59, n. 3, p. 817-858, 1991.

BAI, J.; NG, S. Determining the number of factors in approximate factor models. Econometrica, Wiley Online Library, v. 70, n. 1, p. 191-221, 2002.

. Forecasting economic time series using targeted predictors. Journal of Econometrics, Elsevier, v. 146, n. 2, p. 304-317, 2008.

BAŃBURA, M.; GIANNONE, D.; REICHLIN, L. Large Bayesian VARs. [S.I.], 2008.

BARIGOZZI, M.; HALLIN, M. Generalized dynamic factor models and volatilities: Consistency, rates, and prediction intervals. Journal of Econometrics, Elsevier, 2020.

BERNARDI, M.; CATANIA, L. The model confidence set package for r. International Journal of Computational Economics and Econometrics, v. 8, n. 2, p. 144-158, 2018.

BICKEL, P. J.; LEVINA, E. et al. Covariance regularization by thresholding. The Annals of Statistics, Institute of Mathematical Statistics, v. 36, n. 6, p. 2577-2604, 2008.

BOIVIN, J.; NG, S. Are more data always better for factor analysis? Journal of Econometrics, Elsevier, v. 132, n. 1, p. 169-194, 2006.

BRILLINGER, D. R. Time series: data analysis and theory. [S.l.]: Siam, 1981. v. 36.

CASTLE, J. L.; CLEMENTS, M. P.; HENDRY, D. F. Forecasting by factors, by variables, by both or neither? Journal of Econometrics, Elsevier, v. 177, n. 2, p. 305-319, 2013.

CLEMENTS, M.; HENDRY, D. Forecasting economic time series. [S.l.]: Cambridge University Press, 1998.

EKLUND, J.; KAPETANIOS, G. A review of forecasting techniques for large data sets. National Institute Economic Review, Sage Publications Sage UK: London, England, v. 203, n. 1, p. 109-115, 2008.

FAN, J.; LIAO, Y. Learning latent factors from diversified projections and its applications to over-estimated and weak factors. Available at SSRN 3446097, 2019.

FAN, J.; LIAO, Y.; LIU, H. An overview of the estimation of large covariance and precision matrices. The Econometrics Journal, Wiley Online Library, v. 19, n. 1, p. C1-C32, 2016.

FAN, J.; WANG, W.; ZHONG, Y. Robust covariance estimation for approximate factor models. Journal of econometrics, Elsevier, v. 208, n. 1, p. 5-22, 2019. 
FORNI, M.; GIANNONE, D.; LIPPI, M.; REICHLIN, L. Opening the black box: Structural factor models with large cross sections. Econometric Theory, Cambridge University Press, v. 25, n. 5, p. 1319-1347, 2009.

FORNI, M.; HALLIN, M.; LIPPI, M.; REICHLIN, L. The generalized dynamic-factor model: Identification and estimation. Review of Economics and statistics, MIT Press, v. 82, n. 4, p. 540-554, 2000.

. The generalized dynamic factor model: one-sided estimation and forecasting. Journal of the American Statistical Association, Taylor \& Francis, v. 100, n. 471, p. 830-840, 2005.

FORNI, M.; LIPPI, M. The general dynamic factor model: One-sided representation results. Journal of Econometrics, Elsevier, v. 163, n. 1, p. 23-28, 2011.

FRIEDMAN, J.; HASTIE, T.; TIBSHIRANI, R. The elements of statistical learning. [S.l.]: Springer series in statistics New York, 2001. v. 1.

GIANNONE, D.; REICHLIN, L.; SALA, L. Monetary policy in real time. NBER macroeconomics annual, MIT Press, v. 19, p. 161-200, 2004.

GROEN, J. J.; KAPETANIOS, G. Revisiting useful approaches to data-rich macroeconomic forecasting. Computational Statistics \& Data Analysis, Elsevier, v. 100, p. 221-239, 2016.

HALLIN, M.; LISKA, R. et al. The generalized dynamic factor model: determining the number of factors. Journal of the American Statistical Association, v. 102, n. 478, p. 603-617, 2007.

HANSEN, P. R.; LUNDE, A.; NASON, J. M. The model confidence set. Econometrica, Wiley Online Library, v. 79, n. 2, p. 453-497, 2011.

HOERL, A. E.; KENNARD, R. W. Ridge regression: Biased estimation for nonorthogonal problems. Technometrics, Taylor \& Francis Group, v. 12, n. 1, p. 55-67, 1970.

JOHNSON, R. A.; WICHERN, D. W. et al. Applied multivariate statistical analysis. [S.l.]: Prentice hall Upper Saddle River, NJ, 2002. v. 5.

JOLLIFFE, I. Principal Component Analysis. [S.l.]: Springer-Verlag, 1986.

KIEFER, N. M.; VOGELSANG, T. J. Heteroskedasticity-autocorrelation robust standard errors using the bartlett kernel without truncation. Econometrica, Wiley Online Library, v. 70, n. 5, p. 2093-2095, 2002.

. A new asymptotic theory for heteroskedasticity-autocorrelation robust tests. Econometric Theory, Cambridge University Press, v. 21, n. 6, p. 1130-1164, 2005.

KIM, H. H.; SWANSON, N. R. Forecasting financial and macroeconomic variables using data reduction methods: New empirical evidence. Journal of Econometrics, Elsevier, v. 178, p. 352-367, 2014.

KOOP, G. M. Forecasting with medium and large Bayesian VARs. Journal of Applied Econometrics, Wiley Online Library, v. 28, n. 2, p. 177-203, 2013.

KRISTENSEN, J. T. Diffusion indexes with sparse loadings. Journal of Business \& Economic Statistics, Taylor \& Francis, v. 35, n. 3, p. 434-451, 2017. 
LAURINI, M. P.; OHASHI, A. A noisy principal component analysis for forward rate curves. European Journal of Operational Research, Elsevier, v. 246, n. 1, p. 140-153, 2015.

LEDOIT, O.; WOLF, M. A well-conditioned estimator for large-dimensional covariance matrices. Journal of multivariate analysis, Elsevier, v. 88, n. 2, p. 365-411, 2004.

MEDEIROS, M. C.; VASCONCELOS, G.; FREITAS, E. Forecasting Brazilian inflation with high-dimensional models. Brazilian Review of Econometrics, v. 36, n. 2, p. 223-254, 2016.

MÜLLER, U. K. A theory of robust long-run variance estimation. Journal of Econometrics, Elsevier, v. 141, n. 2, p. 1331-1352, 2007.

ONATSKI, A. Determining the number of factors from empirical distribution of eigenvalues. The Review of Economics and Statistics, MIT Press, v. 92, n. 4, p. 1004-1016, 2010.

POURAHMADI, M. High-dimensional covariance estimation: with high-dimensional data. [S.l.]: John Wiley \& Sons, 2013. v. 882.

SMEEKES, S.; WIJLER, E. Macroeconomic forecasting using penalized regression methods. International Journal of Forecasting, Elsevier, v. 34, n. 3, p. 408-430, 2018.

STOCK, J. H.; WATSON, M. Forecasting in dynamic factor models subject to structural instability. The Methodology and Practice of Econometrics. A Festschrift in Honour of David F. Hendry, Oxford University Press Oxford, v. 173, p. 205, 2009.

STOCK, J. H.; WATSON, M. W. Forecasting using principal components from a large number of predictors. Journal of the American Statistical Association, Taylor \& Francis, v. 97, n. 460, p. 1167-1179, 2002.

. Generalized shrinkage methods for forecasting using many predictors. Journal of Business \& Economic Statistics, Taylor \& Francis, v. 30, n. 4, p. 481-493, 2012.

. Factor models and structural vector autoregressions in macroeconomics. In: Handbook of Macroeconomics. [S.l.]: Elsevier, 2016. v. 2, p. 415-526.

TIBSHIRANI, R. Regression shrinkage and selection via the lasso. Journal of the Royal Statistical Society. Series B (Methodological), JSTOR, p. 267-288, 1996.

TIBSHIRANI, R.; WAINWRIGHT, M.; HASTIE, T. Statistical learning with sparsity: the lasso and generalizations. [S.l.]: Chapman and Hall/CRC, 2015.

VIEIRA, F; FERNANDES, M.; CHAGUE, F. Forecasting the Brazilian yield curve using forward-looking variables. International Journal of Forecasting, Elsevier, v. 33, n. 1, p. 121-131, 2017.

WANG, H.; LI, B.; LENG, C. Shrinkage tuning parameter selection with a diverging number of parameters. Journal of the Royal Statistical Society: Series B (Statistical Methodology), Wiley Online Library, v. 71, n. 3, p. 671-683, 2009.

ZEILEIS, A. Econometric computing with hc and hac covariance matrix estimators. Institut für Statistik und Mathematik, WU Vienna University of Economics and ..., 2004.

ZOU, H.; HASTIE, T. Regularization and variable selection via the elastic net. Journal of the royal statistical society: series B (statistical methodology), Wiley Online Library, v. 67, n. 2, p. 301-320, 2005. 
ZOU, H.; HASTIE, T.; TIBSHIRANI, R. Sparse principal component analysis. Journal of Computational and Graphical Statistics, Taylor \& Francis, v. 15, n. 2, p. 265-286, 2006. 
APÊNDICES 



\section{APÊNDICE A - MSFE PARA CADA MODELO ESTIMADO}

\section{A.1 GDP251}

\begin{tabular}{cccccc}
\hline & FH1 & FH2 & FH4 & FH6 & FH8 \\
\hline M01PC & 0.3454 & 0.2376 & 0.1915 & 0.1801 & 0.0772 \\
M02PC & 0.1696 & 0.3178 & 0.2861 & 0.1517 & 0.0831 \\
M03PC & 0.3725 & 0.4370 & 0.3406 & 0.3578 & 0.1414 \\
M04PC & 0.3558 & 0.3485 & 0.2785 & 0.2833 & 0.1070 \\
M05PC & 0.4125 & 0.4001 & 0.3792 & 0.1963 & 0.0911 \\
M06PC & 0.3456 & 0.2393 & 0.1908 & 0.1804 & 0.0774 \\
M07PC & 0.1622 & 0.3137 & 0.2916 & 0.1486 & 0.0868 \\
M01SPC & 0.3455 & 0.2376 & 0.1915 & 0.1801 & 0.0772 \\
M02SPC & 0.3505 & 0.2593 & 0.1925 & 0.1852 & 0.0780 \\
M03SPC & 0.3403 & 0.2412 & 0.1905 & 0.1814 & 0.0777 \\
M04SPC & 0.3446 & 0.4260 & 0.3783 & 0.3889 & 0.1625 \\
M05SPC & 0.2907 & 0.3797 & 0.3787 & 0.3736 & 0.1699 \\
M06SPC & 0.3358 & 0.4222 & 0.4073 & 0.4034 & 0.1773 \\
M07SPC & 0.3528 & 0.3492 & 0.2843 & 0.2908 & 0.1116 \\
M08SPC & 0.3171 & 0.3718 & 0.3448 & 0.3479 & 0.1465 \\
M09SPC & 0.3538 & 0.3531 & 0.2968 & 0.3017 & 0.1184 \\
M10SPC & 0.3457 & 0.2392 & 0.1903 & 0.1803 & 0.0774 \\
M11SPC & 0.3466 & 0.2391 & 0.1892 & 0.1816 & 0.0776 \\
M12SPC & 0.3459 & 0.2389 & 0.1899 & 0.1805 & 0.0775 \\
M01CONC & 0.3486 & 0.4325 & 0.7376 & 0.5861 & 0.6096 \\
M02CONC & 0.3461 & 0.3742 & 1.2048 & 1.0530 & 1.2347 \\
M03CONC & 0.2440 & 0.2485 & 0.2068 & 0.1503 & 0.0745 \\
M04CONC & 0.1953 & 0.2154 & 0.2586 & 0.1486 & 0.0834 \\
\hline
\end{tabular}

Tabela A.1 - MSFE para todos os modelos estimados na aplicação empírica para o GDP251 


\section{A.2 IPS10}

\begin{tabular}{cccccc}
\hline & FH1 & FH2 & FH4 & FH6 & FH8 \\
\hline M01PC & 0.2423 & 0.2262 & 0.0826 & 0.0820 & 0.0831 \\
M02PC & 0.1439 & 0.2335 & 0.2060 & 0.1546 & 0.0797 \\
M03PC & 0.2738 & 0.2989 & 0.2147 & 0.2133 & 0.1917 \\
M04PC & 0.2567 & 0.2757 & 0.1337 & 0.1372 & 0.1427 \\
M05PC & 0.2444 & 0.2665 & 0.1499 & 0.1468 & 0.0922 \\
M06PC & 0.2425 & 0.2267 & 0.0828 & 0.0816 & 0.0831 \\
M07PC & 0.1388 & 0.2297 & 0.1965 & 0.1552 & 0.0785 \\
M01SPC & 0.2422 & 0.2262 & 0.0826 & 0.0820 & 0.0831 \\
M02SPC & 0.2500 & 0.2429 & 0.0885 & 0.0800 & 0.0827 \\
M03SPC & 0.2428 & 0.2270 & 0.0828 & 0.0808 & 0.0832 \\
M04SPC & 0.2688 & 0.3042 & 0.2165 & 0.2095 & 0.2480 \\
M05SPC & 0.2085 & 0.2173 & 0.1977 & 0.2360 & 0.2566 \\
M06SPC & 0.2544 & 0.2917 & 0.2235 & 0.2357 & 0.2613 \\
M07SPC & 0.2558 & 0.2749 & 0.1366 & 0.1422 & 0.1482 \\
M08SPC & 0.2280 & 0.2574 & 0.1655 & 0.1830 & 0.1998 \\
M09SPC & 0.2585 & 0.2810 & 0.1423 & 0.1519 & 0.1564 \\
M10SPC & 0.2431 & 0.2274 & 0.0825 & 0.0814 & 0.0831 \\
M11SPC & 0.2464 & 0.2284 & 0.0826 & 0.0814 & 0.0831 \\
M12SPC & 0.2436 & 0.2276 & 0.0824 & 0.0814 & 0.0830 \\
M01CONC & 0.3831 & 0.2784 & 2.9777 & 1.6082 & 1.3842 \\
M02CONC & 0.7629 & 0.3737 & 4.5558 & 2.4931 & 2.1348 \\
M03CONC & 0.1744 & 0.1819 & 0.2104 & 0.1218 & 0.1194 \\
M04CONC & 0.1281 & 0.1320 & 0.1714 & 0.1496 & 0.0911 \\
\hline
\end{tabular}

Tabela A.2 - MSFE para todos os modelos estimados na aplicação empírica para o IPS10 


\section{A.3 LHUR}

\begin{tabular}{cccccc}
\hline & FH1 & FH2 & FH4 & FH6 & FH8 \\
\hline M01PC & 0.2598 & 0.2983 & 0.2501 & 0.2450 & 0.2859 \\
M02PC & 0.1148 & 0.1328 & 0.2172 & 0.2233 & 0.2159 \\
M03PC & 0.3525 & 0.4846 & 0.5171 & 0.5460 & 0.5867 \\
M04PC & 0.3385 & 0.4173 & 0.4034 & 0.4220 & 0.4800 \\
M05PC & 0.2416 & 0.3239 & 0.3068 & 0.2576 & 0.2108 \\
M06PC & 0.2603 & 0.2984 & 0.2505 & 0.2450 & 0.2864 \\
M07PC & 0.1130 & 0.1326 & 0.2046 & 0.2242 & 0.2157 \\
M01SPC & 0.2597 & 0.2982 & 0.2501 & 0.2449 & 0.2859 \\
M02SPC & 0.2586 & 0.3143 & 0.2584 & 0.2447 & 0.2916 \\
M03SPC & 0.2593 & 0.2974 & 0.2509 & 0.2451 & 0.2867 \\
M04SPC & 0.3448 & 0.4713 & 0.5487 & 0.5695 & 0.6054 \\
M05SPC & 0.3091 & 0.4105 & 0.5213 & 0.5825 & 0.5713 \\
M06SPC & 0.3384 & 0.4728 & 0.5659 & 0.6156 & 0.6338 \\
M07SPC & 0.3407 & 0.4218 & 0.4110 & 0.4333 & 0.4962 \\
M08SPC & 0.3243 & 0.4203 & 0.4731 & 0.5209 & 0.5933 \\
M09SPC & 0.3437 & 0.4307 & 0.4247 & 0.4546 & 0.5186 \\
M10SPC & 0.2609 & 0.2985 & 0.2502 & 0.2449 & 0.2865 \\
M11SPC & 0.2611 & 0.3011 & 0.2503 & 0.2449 & 0.2868 \\
M12SPC & 0.2609 & 0.2992 & 0.2500 & 0.2448 & 0.2865 \\
M01CONC & 0.9921 & 0.3700 & 0.6107 & 6.2351 & 0.2924 \\
M02CONC & 0.8312 & 0.4238 & 0.6266 & 8.0102 & 0.4289 \\
M03CONC & 0.1955 & 0.1692 & 0.2599 & 0.2494 & 0.2773 \\
M04CONC & 0.3471 & 0.3611 & 0.2691 & 0.2236 & 0.3141 \\
\hline
\end{tabular}

Tabela A.3 - MSFE para todos os modelos estimados na aplicação empírica para o LHUR 


\section{A.4 CPIAUCSL}

\begin{tabular}{cccccc}
\hline & FH1 & FH2 & FH4 & FH6 & FH8 \\
\hline M01PC & 1.0779 & 1.1744 & 1.2536 & 1.4697 & 1.8380 \\
M02PC & 1.3620 & 1.2095 & 1.3222 & 1.5080 & 1.8398 \\
M03PC & 1.3099 & 1.2183 & 1.3244 & 1.5458 & 1.8306 \\
M04PC & 1.2669 & 1.2472 & 1.2921 & 1.4872 & 1.8209 \\
M05PC & 1.2847 & 1.2119 & 1.3650 & 1.4234 & 1.9122 \\
M06PC & 1.0745 & 1.1752 & 1.2527 & 1.4692 & 1.8378 \\
M07PC & 1.1478 & 1.2533 & 1.3525 & 1.4939 & 1.8397 \\
M01SPC & 1.0779 & 1.1744 & 1.2536 & 1.4697 & 1.8381 \\
M02SPC & 1.0620 & 1.1864 & 1.2590 & 1.4688 & 1.8394 \\
M03SPC & 1.0731 & 1.1767 & 1.2532 & 1.4687 & 1.8384 \\
M04SPC & 1.2847 & 1.2073 & 1.3486 & 1.5300 & 1.8921 \\
M05SPC & 1.2065 & 1.1970 & 1.3749 & 1.5505 & 1.9223 \\
M06SPC & 1.2834 & 1.2172 & 1.3665 & 1.5452 & 1.8959 \\
M07SPC & 1.2478 & 1.2448 & 1.2904 & 1.4897 & 1.8229 \\
M08SPC & 1.1537 & 1.2439 & 1.3228 & 1.5065 & 1.8278 \\
M09SPC & 1.2430 & 1.2496 & 1.2949 & 1.4924 & 1.8275 \\
M10SPC & 1.0737 & 1.1760 & 1.2523 & 1.4688 & 1.8378 \\
M11SPC & 1.0781 & 1.1756 & 1.2529 & 1.4694 & 1.8381 \\
M12SPC & 1.0741 & 1.1761 & 1.2523 & 1.4688 & 1.8378 \\
M01CONC & 1.4749 & 1.3007 & 0.9693 & 5.6704 & 1.8098 \\
M02CONC & 1.8633 & 1.3822 & 0.9320 & 6.5534 & 2.1865 \\
M03CONC & 1.1735 & 1.1538 & 1.2301 & 1.6907 & 1.7400 \\
M04CONC & 0.9364 & 1.1203 & 1.2344 & 1.5326 & 1.8214 \\
\hline
\end{tabular}

Tabela A.4 - MSFE para todos os modelos estimados na aplicação empírica para o CPIAUCSL 


\section{A.5 FSPCOM}

\begin{tabular}{cccccc}
\hline & FH1 & FH2 & FH4 & FH6 & FH8 \\
\hline M01PC & 1.2079 & 1.3744 & 0.4327 & 0.3709 & 0.2926 \\
M02PC & 1.5258 & 1.6784 & 0.4507 & 0.3788 & 0.3070 \\
M03PC & 1.3133 & 1.5370 & 0.4245 & 0.2962 & 0.2736 \\
M04PC & 1.2970 & 1.5345 & 0.4280 & 0.3022 & 0.2706 \\
M05PC & 1.4651 & 1.7284 & 0.6112 & 0.2753 & 0.3284 \\
M06PC & 1.2077 & 1.3742 & 0.4328 & 0.3710 & 0.2927 \\
M07PC & 1.4685 & 1.6302 & 0.4107 & 0.3754 & 0.3117 \\
M01SPC & 1.2079 & 1.3744 & 0.4327 & 0.3709 & 0.2926 \\
M02SPC & 1.2068 & 1.3738 & 0.4328 & 0.3710 & 0.2926 \\
M03SPC & 1.2077 & 1.3735 & 0.4326 & 0.3713 & 0.2927 \\
M04SPC & 1.3126 & 1.5353 & 0.4253 & 0.2950 & 0.2727 \\
M05SPC & 1.2901 & 1.5127 & 0.4168 & 0.2978 & 0.2798 \\
M06SPC & 1.3275 & 1.5558 & 0.4254 & 0.2808 & 0.2760 \\
M07SPC & 1.3004 & 1.5378 & 0.4276 & 0.2996 & 0.2696 \\
M08SPC & 1.2697 & 1.4963 & 0.4210 & 0.3064 & 0.2694 \\
M09SPC & 1.3040 & 1.5435 & 0.4267 & 0.2954 & 0.2697 \\
M10SPC & 1.2078 & 1.3741 & 0.4328 & 0.3710 & 0.2927 \\
M11SPC & 1.2079 & 1.3745 & 0.4329 & 0.3710 & 0.2926 \\
M12SPC & 1.2078 & 1.3742 & 0.4328 & 0.3710 & 0.2927 \\
M01CONC & 1.8050 & 2.0523 & 1.6043 & 0.7502 & 2.8594 \\
M02CONC & 1.7626 & 2.1713 & 1.8292 & 0.4439 & 3.1552 \\
M03CONC & 1.3373 & 1.5551 & 0.4303 & 0.3706 & 0.2932 \\
M04CONC & 1.2072 & 1.4226 & 0.4568 & 0.3266 & 0.2742 \\
\hline
\end{tabular}

Tabela A.5 - MSFE para todos os modelos estimados na aplicação empírica para o FSPCOM 